NUREG/CR-6426

ORNL-6892

Vol. 1

Ductile Fracture Toughness of

Modified A 302 Grade B Plate

Materials, Data Analysis

RECEIVED

FEB 181997

OSTI

Prepared by

D. E. McCabe, E. T. Manneschmidt, R. L. Swain

Oak Ridge National Laboratory

Prepared for

U.S. Nuclear Regulatory Commission

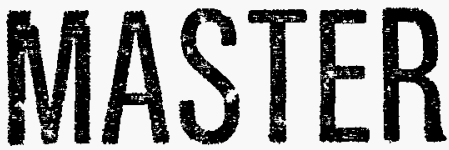

DISTRIBUTION! OF THIS DOCUMENT IS UNLIAITED 


\section{AVAILABILITY NOTICE}

\section{Availability of Reference Materials Cited in NRC Publications}

Most documents cited in NRC publications will be availabie from one of the following sources:

1. The NRC Public Document Room, 2120 L Street, NW., Lower Level, Washington, DC 20555-0001

2. The Superintendent of Documents, U.S. Government Printing Office, P. O. Box 37082 , Washington, DC 20402-9328

3. The National Technical Information Service, Springfield, VA 22161-0002

Although the listing that follows represents the majority of documents cited in NRC publications, it is not intended to be exhaustlve.

Referenced documents available for inspection and copying for a fee from the NRC Public Document Room include NRC correspondence and internal NRC memoranda; NRC bulletins, circulars, information notices, inspection and investigation notices; licensee event reports; vendor reports and correspondence; Commission papers: and applicant and licensee documents and correspondence.

The following documents in the NUREG series are available for purchase from the Government Printing Office: formal NRC staff and contractor reports, NRC-sponsored conference proceedings, international agreement reports, grantee reports, and NRC booklets and brochures. Also available are regulatory guides, NRC regulations in the Code of Federal Regulations, and Nuclear Regulatory Commission Issuances.

Documents avallable from the National Technical Information Service include NUREG-series reports and technical reports prepared by other Federal agencies and reports prepared by the Atomic Energy Commission, forerunner agency to the Nuclear Regulatory Commission.

Documents available from public and special technical libraries include all open literature items, such as books, journal articles, and transactions. Federal Register notices. Federal and State legislation, and congressional reports can usually be obtained from these libraries.

Documents such as theses, dissertations, foreign reports and translations, and non-NRC conference proceedings are available for purchase from the organization sponsoring the publication cited.

Single coples of NRC draft reports are available free, to the extent of supply, upon written request to the Office of Administration, Distribution and Mail Services Section, U.S. Nuclear Regulatory Commission, Washington, DC 20555-0001.

Copies of Industry codes and standards used in a substantive manner in the NRC regulatory process are maintained at the NRC Library, Two White Flint North, 11545 Rockville Pike. Rockville. MD 20852-2738. for use by the public. Codes and standards are usually copyrighted and may be purchased from the originating organization or, if they are American National Standards. from the American National Standards Institute. 1430 Broadway, New York, NY 10018-3308.

\section{DISCLAIMER NOTICE}

This report was prepared as an account of work sponsored by an agency of the United States Govemment. Neither the United States Government nor any agency thereof, nor any of their employees, makes any warranty, expressed or implied, or assumes any legal liability or responsibility for any third party's use, or the results of such use, of any information, apparatus, product, or process disclosed in this report, or represents that its use by such third party would not infringe privately owned rights. 
NUREG/CR-6426

ORNL-6892

Vol. 1

\section{Ductile Fracture Toughness of Modified A 302 Grade B Plate Materials, Data Analysis}

Manuscript Completed: September 1996

Date Published: January 1997

Prepared by

D. E. McCabe, E. T. Manneschmidt, R. L. Swain

Oak Ridge National Laboratory

Managed by Lockheed Martin Energy Research Corporation

Oak Ridge National Laboratory

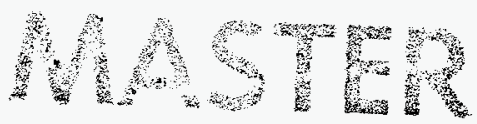

Oak Ridge, TN 37831-6285

S. N. M. Malik, NRC Project Manager

Prepared for

Division of Engineering Technology

Office of Nuclear Regulatory Research

U.S. Nuclear Regulatory Commission

Washington, DC 20555-0001

NRC Job Code B0119 


\section{DISCLAMMER}

Portions of this document may be illegible in electronic image products. Images are produced from the best available original document. 


\begin{abstract}
The objective of this work was to develop ductile fracture toughness data in the form of J-R curves for modified A 302 grade $B$ plate materials typical of those used in fabricating reactor pressure vessels. A previous experimental study at Materials Engineering Associates (MEA), Lanham, Maryland, on one particular heat of A 302 grade B plate showed decreasing $J-R$ curves with increased specimen thickness. This characteristic has not been observed in numerous tests made on the more recent production materials of $A 533$ grade $B$ and $A 508$ class 2 pressure vessel steels. It was unknown if the departure from norm for the MEA material was a generic characteristic for all heats of A 302 grade $B$ steels or just unique to that one particular plate.

Seven heats of modified A 302 grade B steel and one heat of vintage A 533 grade B steel were provided to this project by the General Electric Company of San Jose, California. All plates were tested for chemical content, tensile properties, Charpy transition temperature curves, drop-weight nil-ductility transition (NDT) temperature, and $J-R$ curves. Tensile tests were made in the three principal orientations and at four temperatures, ranging from room temperature to $550^{\circ} \mathrm{F}\left(288^{\circ} \mathrm{C}\right)$. Charpy $\mathrm{V}$-notch transition temperature curves were obtained in longitudinal, transverse, and short transverse orientations. J-R curves were made using four specimen sizes (1/2T, 1T, 2T, and $4 T$ ). The fracture mechanics-based evaluation method covered three test orientations and three test temperatures $\left[180,400\right.$, and $550^{\circ} \mathrm{F}\left(82,204\right.$, and $\left.\left.288^{\circ} \mathrm{C}\right)\right]$. However, the coverage of these variables was contingent upon the amount of material provided. Drop-weight NDT temperature was determined for the T-L orientation only.
\end{abstract}

None of the seven heats of modified A 302 grade B showed size effects of any consequence on the J-R curve behavior. Crack orientation effects were present, but none were severe enough to be reported as atypical. A test temperature increase from 180 to $550^{\circ} \mathrm{F}\left(82\right.$ to $\left.288^{\circ} \mathrm{C}\right)$ produced the usual loss in J-R curve fracture toughness. Generic J-R curves and mathematical curve fits to the same were generated to represent each heat of material.

This volume (Nol. 1) deals with the evaluation of data and the discussion of technical findings. Volume 2 is a compilation of all data developed. 



\section{Contents}

Page

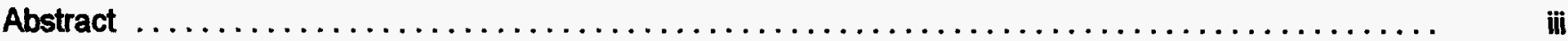

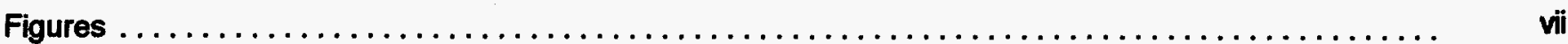

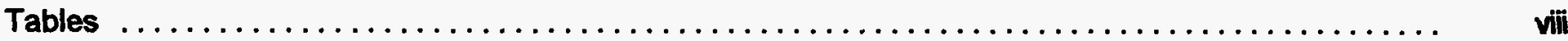

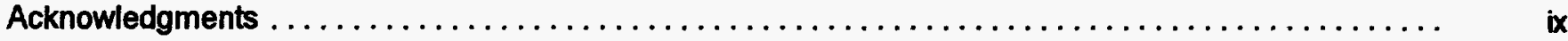

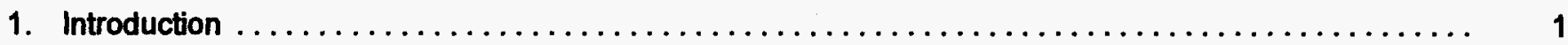

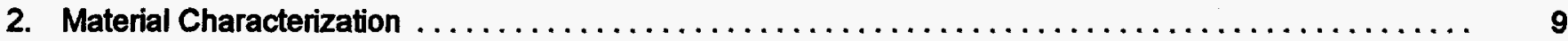

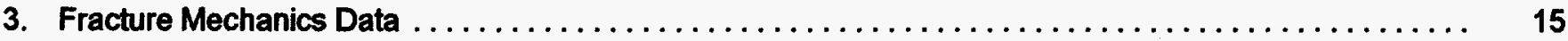

4. J-R Curve Characteristics of Seven Heats of Modified A 302 Grade B Steel . . . . . . . . . . . . . . . 17

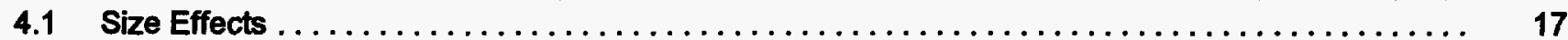

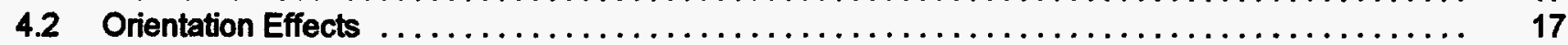

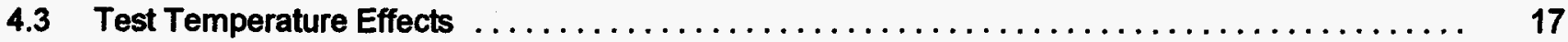

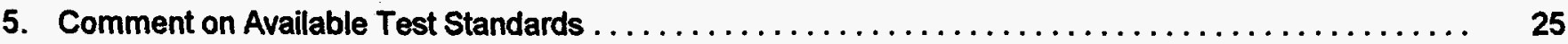

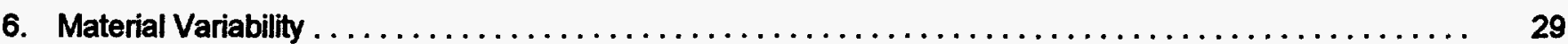

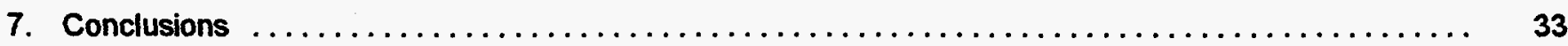

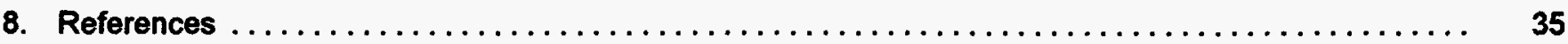

Appendix A: Selected J-R Curves Representing Typical Values of T Slope, $J_{0.1}$, and $J_{k}$ from Table $7 \quad \ldots \ldots$ A-1

Appendix B: Selected J-R Curves Representing Typical Values of T Slope, $J_{0.1}$, and $J_{k}$ from Table $8 \ldots \ldots$ B-1 



\section{Figures}

Page

$1 \mathrm{~J}$-R curve for two $1 \mathrm{~T}$ compact specimens tested at $180^{\circ} \mathrm{F}\left(82^{\circ} \mathrm{C}\right)$, showing J-integral by deformation theory J and modified J. Source: A. L. Hiser and J. B. Terrell, Material Engineering Associates, Inc., Lanham, Md., Size Effects on J-R Curves for A302-B Plate, USNRC Report NUREG/CR-6265 (MEA-2320), January 1989

$2 \mathrm{~J}$-R curve for one $6 \mathrm{~T}$ compact specimen tested at $180^{\circ} \mathrm{F}\left(82^{\circ} \mathrm{C}\right)$, showing J-integral by modified J. Source: A. L. Hiser and J. B. Terrell, Material Engineering Associates, Inc., Lanham, Md., Size Effects on J-R Curves for A302-B Plate, USNRC Report NUREG/CR-6265

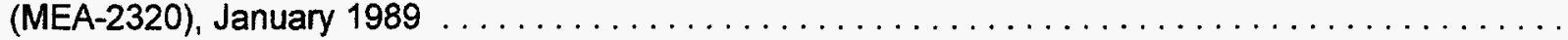

3 General Electric-supplied plates of modified A 302 grade B steel: (a) Codes Z1 and Z2,

(b) Codes Z3 and Z4, (c) Codes Z5 and Z6, and (d) Codes Z7 and Z8

4 Schematic of J-R curve plot and measurements taken. Filled data points represent the points used in the power law curve fit.

$5 \mathrm{~J}-\mathrm{R}$ curves from compact specimens of varied size for the typical modified A 302 grade B steel versus the Materials Engineering Associates plate V50 of A 302 grade $B$ steel . . . . . . . . . .

6 Load-displacement record of $1 / 2 \mathrm{~T}$ compact specimen tested at $180^{\circ} \mathrm{F}\left(82^{\circ} \mathrm{C}\right)$, showing no evidence of pop-in . . . . . . . . . . . . . . . . . . . . . . . . . . . . . . . . . . .

7 Load-displacement record of $1 / 2 \mathrm{~T}$ compact specimen tested at $400^{\circ} \mathrm{F}\left(204^{\circ} \mathrm{C}\right)$, showing no evidence of pop-in

8 Load-displacement record of $1 / 2 \mathrm{~T}$ compact specimen tested at $550^{\circ} \mathrm{F}\left(288^{\circ} \mathrm{C}\right)$, showing a hint of

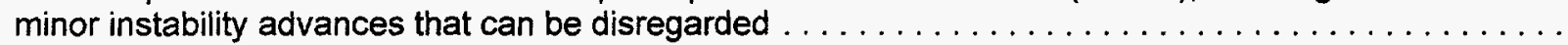

9 Load-displacement record of $2 \mathrm{~T}$ compact specimen tested at $180^{\circ} \mathrm{F}\left(82^{\circ} \mathrm{C}\right)$, an upper-shelf temperature with no evidence of pop-in .

10 Load-displacement record of $2 \mathrm{~T}$ compact specimen tested at $550^{\circ} \mathrm{F}\left(288^{\circ} \mathrm{C}\right)$, showing clear evidence of pop-in behavior at upper-shelf temperature

11 Comparison of the first American Society for Testing and Materials test standard on $J_{\mathbb{~}}$ in 1981 to the first revision made in 1989

12 The 1996 American Society for Testing and Materials method (combined standard):

(a) third-order polynomial fit and $(b)$ adjusted $J_{R}-\Delta$ a data to the origin at the known initial physical crack size of the specimen

13 Comparison of the 1989 American Society for Testing and Materials $\mathrm{J}_{\mathrm{Ic}}$ test method revision to the

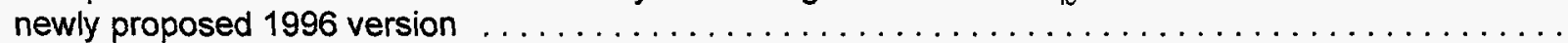

14 Data scatter of $J_{I c}$ due to material variability

15 Typical J-R curve for comparison to the duplicate test shown in Figure $16 \ldots \ldots \ldots \ldots \ldots \ldots$

16 Typical J-R curve for comparison to the duplicate test shown in Figure 15.

17 Data scatter of $J_{0.1}$ due to material variability 
$18 \mathrm{~J}-\mathrm{R}$ curves for modified A302 grade B plates, one vintage A533 grade B class 1 plate (Z8) and a Materials Engineering Associates plate of A302 grade B (V50). Tensile properties and

corresponding Charpy $V$-notch impact upper-shelf energies $(\mathrm{ft}-\mathrm{lb})$ are also shown

\section{Tables}

1 Fracture toughness of modified $\mathrm{A} 302$ grade $B$ - test matrix $\ldots \ldots \ldots \ldots \ldots \ldots \ldots \ldots \ldots \ldots \ldots$

2 Modified A 302 grade B test materials, heat code, and chemistries $\ldots \ldots \ldots \ldots \ldots \ldots \ldots \ldots \ldots$

3 Tensile properties for seven heats of modified A 302 grade B (Z1-Z7) and one vintage heat of A 533 grade B material (Z8).

4 Reference temperature determinations

5 Charpy V-notch values for material transition temperature and upper-shelf evaluation

6 Evaluation of specimen size on J-R curves of modified A 302 grade B steel [averaged values;

$T-L$ (transverse) orientation and $180^{\circ} \mathrm{F}\left(82^{\circ} \mathrm{C}\right)$; all specimens $20 \%$ side grooved]

7 Directionality of J-R curve fracture toughness at $180^{\circ} \mathrm{F}$ (averaged values over all specimen sizes; all specimens $20 \%$ side grooved)

8 Test temperature effect on J-R curve [averaged values over all specimen sizes; T-L (transverse) orientation; all specimens $20 \%$ side grooved] 


\section{Acknowledgments}

The authors would like to thank Shah M. Malik, Edwin M. Hackett, and Allen L. Hiser of the U.S. Nuclear Regulatory Commission for their helpful comments in the planning stages of this project. Also, the advice of John G. Merkle of the Engineering Technology Division was invaluable in the planning work.

John J. Henry, Jr., of the Fracture Mechanics Group assisted in this work in many ways, and his contribution is hereby acknowledged. We especially acknowledge Tom Caine of General Electric Nuclear Energy, who was instrumental in providing all the steel plates tested in this project. Thanks also to John G. Merkle and Mikhail A. Sokolov for peer review, to Julia L. Bishop for preparation of the manuscript, and to Kathy Spence for editing and quality assurance review. 


\title{
Ductile Fracture Toughness of Modified A 302 Grade B Plate Materials, Data Analysis*
}

\author{
D. E. McCabe, E. T. Manneschmidt, \\ and R. L. Swain
}

\section{Introduction}

Type A 302 plates of pressure vessel steel are currently in service in about 19 nuclear reactor vessels. ${ }^{1}$ Of these, eight are grade $B$, ten are modified grade $B$, and one is grade A. Type A 302 is an early production version of pressure vessel steel that predates the development of fracture mechanics to a level such that could be applied to ordinary structural steels. Consequently, very little fracture mechanics data have been generated for evaluating the reactor vessels mentioned above. Currently, several reactor vessels are approaching the end of design life, and generic background information is needed to assist with end-of-life and life-extension considerations. This project was initiated to fill this information void by developing $J-R$ curves and the few single-value parameters that can be derived from J-R curves.

A fracture mechanics-based toughness evaluation had been made recently at Materials Engineering Associates (MEA), Lanham, Maryland, that characterized one pedigreed plate of A 302 grade B steel, and these results have at times been used to represent generic $A 302$ grade $B$ performance for in-service evaluations. ${ }^{2}$ The plate was known to have received minimal cross rolling in production, and it was purposely selected for the MEA study because of its low upper-shelf (LUS) fracture toughness in the T-L. (transverse) orientation. The plate had typical A 302 grade $B$ chemistry, with the exception that sulfur and vanadium were a little on the high side. It had received the heat treatment that is normally used on production A 302 grade B plate. The Charpy V-notch (CVN) upper-shelf energy (USE) in the T-L orientation was about $48 \mathrm{ft}-\mathrm{Ib}$ and about $108 \mathrm{ft}-\mathrm{lb}$ in the L-T (longitudinal) orientation. ${ }^{3}$ The expectation in the MEA experiment was that because of the low toughness, the material would not show J-R curve reduction in 1/2T compact specimens. Compact specimens were made of $1 / 2 \mathrm{~T}, 1 \mathrm{~T}, 2 \mathrm{~T}, 4 \mathrm{~T}$, and $6 \mathrm{~T}$ sizes. The testing was confined to one orientation $(T-L)$ and one test temperature $\left[180^{\circ} \mathrm{F}\left(82^{\circ} \mathrm{C}\right)\right]$. Prior to this experiment, the typical J-R curve behavior of steels of more recent production, such as A 533 grade B and A 508 class 2 , showed no differences due to specimen size, with the possible exception of $1 / 2 T$-size compacts that tended to show slightly lower J-R curves, viz., conservative results. Unfortunately, the MEA experiment on A 302 grade B plate showed just the opposite behavior. Both 1/2T and 1T compact specimens showed reasonable J-R curve development comparable to other LUS steels (see Figure 1). On the other hand, the toughness degenerated with increased size to essentially no crack growth resistance increase beyond crack initiation in 6T-size compacts (see Figure 2). Hence, the suggestion is that J-R curves obtained from 1/2T compacts could not be used to represent $A 302$ grade $B$ generically. This pattern reversal presently stands alone as the characterization of the J-R curve behavior of $A 302$ steels.

An objective of this experiment was to generate more generic J-R curve data, except in this case, the material chosen was the more frequently used modified A 302 grade B steel. Seven heats of modified A 302 grade B plate were donated to this investigation by General Electric Company of San Jose, California. All were archival materials that were being saved for possible future tests, as needed, for fracture toughness verification purposes. All represent typical production cross-rolling practices. An add-on to the material selection was one early vintage A 533 grade B plate identified as Z8 in Figure 3(d). The pieces sampled are shown in Figures 3(a) through (d), with the segments supplied to this project marked either as Oak Ridge National Laboratory (ORNL) or MEA.

The test matrix design for J-R curve development encompassed four levels of specimen size, $1 / 2 T, 1 T, 2 T$, and $4 T$; three levels of test temperature, 180,400 , and $550^{\circ} \mathrm{F}\left(82,204\right.$, and $\left.288^{\circ} \mathrm{C}\right)$; and three levels of specimen orientation, T-L, L-T, and L-S (short transverse) [see Table 1]. All conditions were tested in duplicate, except for the

\footnotetext{
"Research was sponsored by the Office of Nuclear Regulatory Research, Division of Engineering Technology, U.S. Nuclear Regulatory Commission, under Interagency Agreement DOE 1886-8011-9B with the U.S. Department of Energy under Contract DE-AC05-96OR22464 with Lockheed Martin Energy Research Corporation.
} 

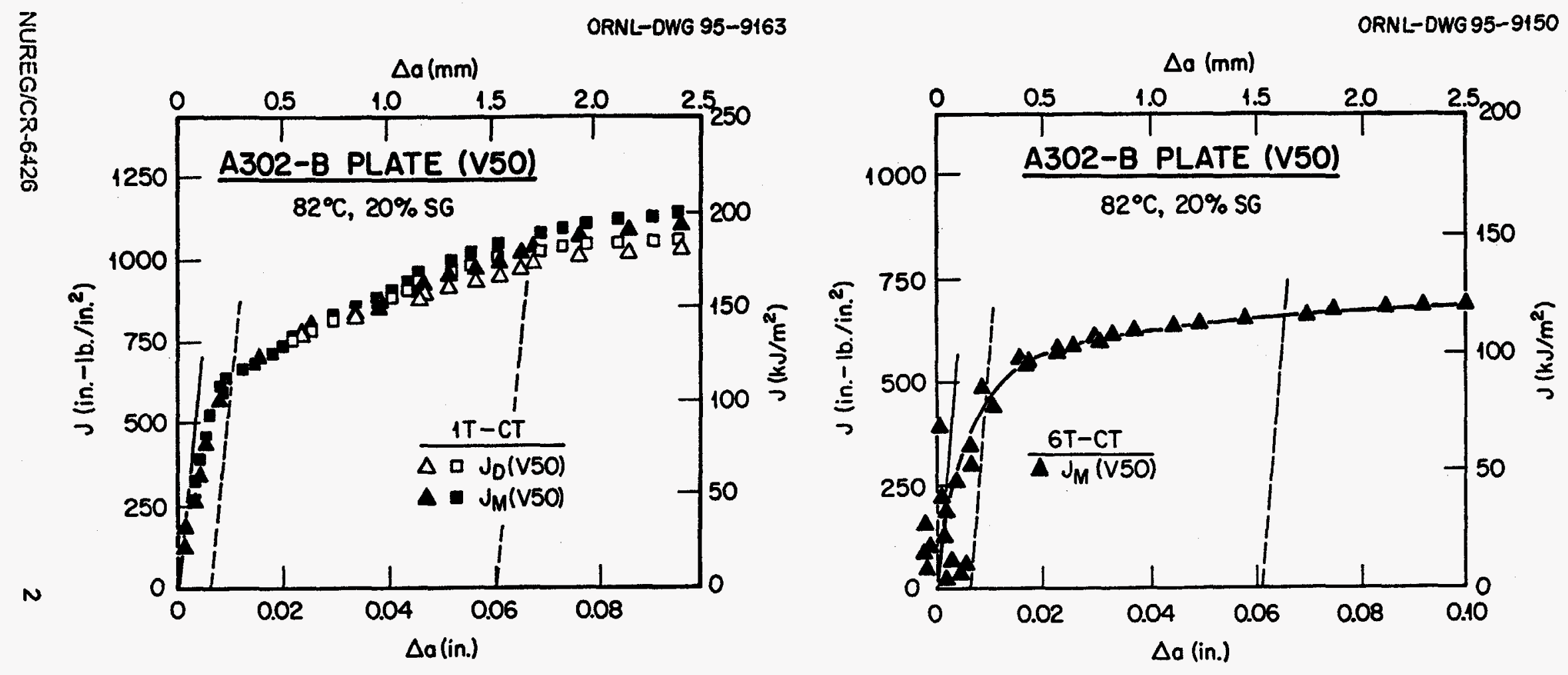

Figure 1. J-R curve for two $1 T$ compact specimens tested at $180^{\circ} \mathrm{F}\left(82^{\circ} \mathrm{C}\right)$, showing J-integral by deformation theory J and modified J. Source: A. L. Hiser and J. B. Terrell, Material Engineering Associates, Inc., Lanham, Md., Size Effects on J-R Cunes for A302-B Plate, USNRC Report NUREG/CR-6265 (MEA-2320), January 1989.

Figure 2. J-R curve for one $6 \mathrm{~T}$ compact specimen tested at $180^{\circ} \mathrm{F}\left(82^{\circ} \mathrm{C}\right)$, showing J-integral by modified J. Source: A. L. Hiser and J. B. Terrell, Material Engineering Associates, Inc., Lanham, Md., Size Effects on J-R Curves for A302-B Plate, USNRC Report NUREG/CR-6265 (MEA-2320), January 1989. 


\section{TEST PLATE CUTTING}

B3990-2
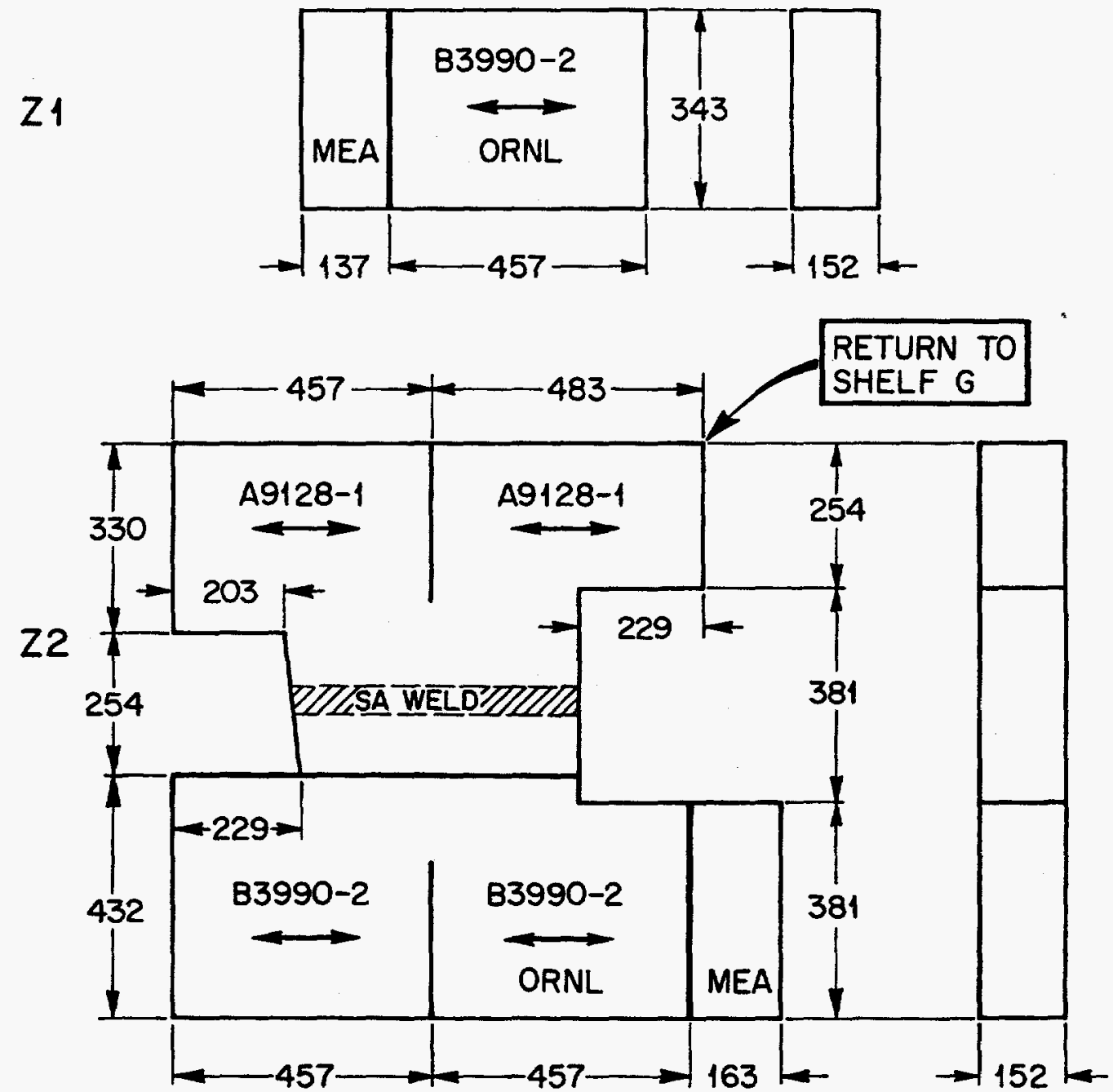

NOTES:

1. ALL DIMENSIONS ARE IN MILLIMETERS

2. ALL EDGES ASSUMED BURNED

Figure 3. General Electric-supplied plates of modified A 302 grade B steel: (a) Codes Z1 and Z2, (b) Codes Z3 and Z4, (c) Codes Z5 and Z6, and $(d)$ Codes Z7 and Z8. 
TEST PLATE CUTTING

A0237-1 or $\mathrm{C} 1256-2$ or $\mathrm{C} 1290-2$

(b)

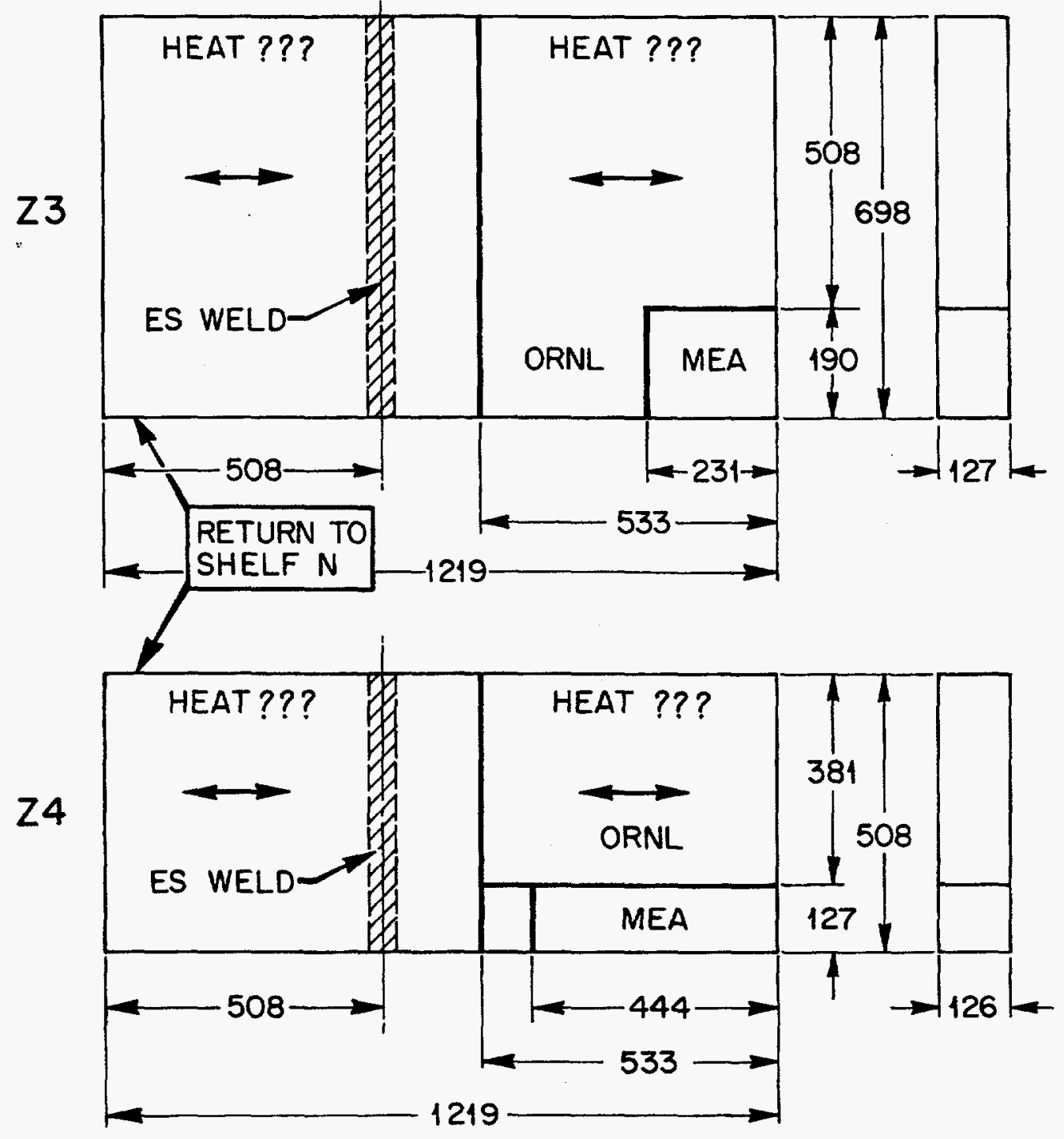

NOTES:

1. ALL DIMENSIONS ARE IN MILLIMETERS

2. ALL EDGES ASSUMED BURNED

Figure 3. (Continued) 


\section{TEST PLATE CUTTING \\ P2130-2}

$\mathrm{Z5}$

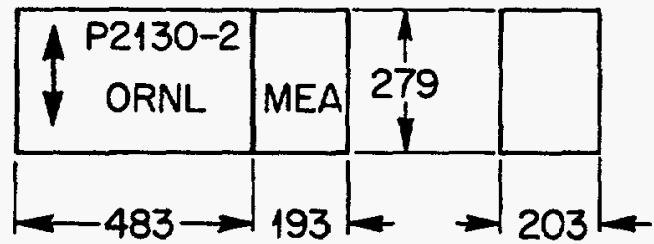

C1079-1 and B5013-2

(c)

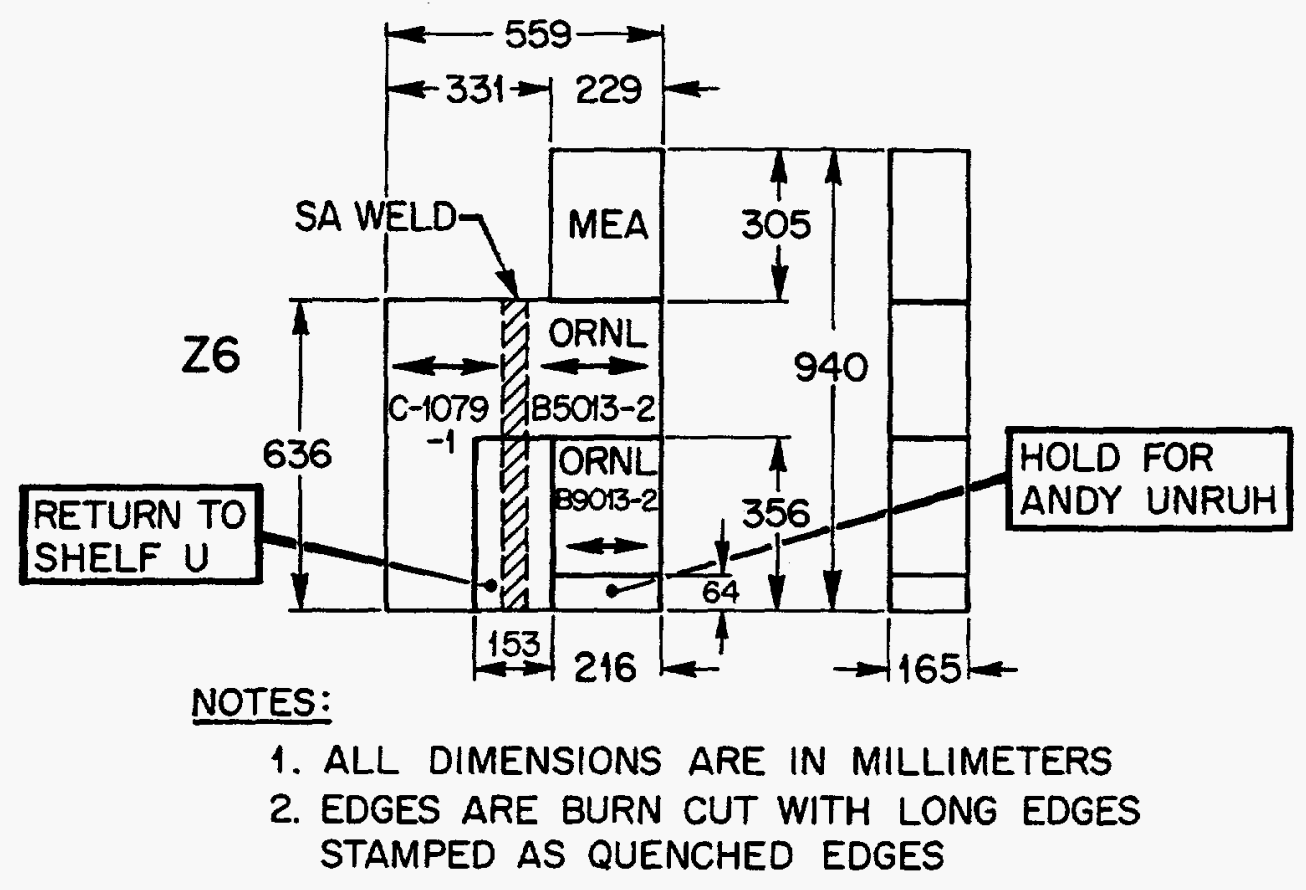

Figure 3. (Continued) 
TEST PLATE CUTTING

c2463-1

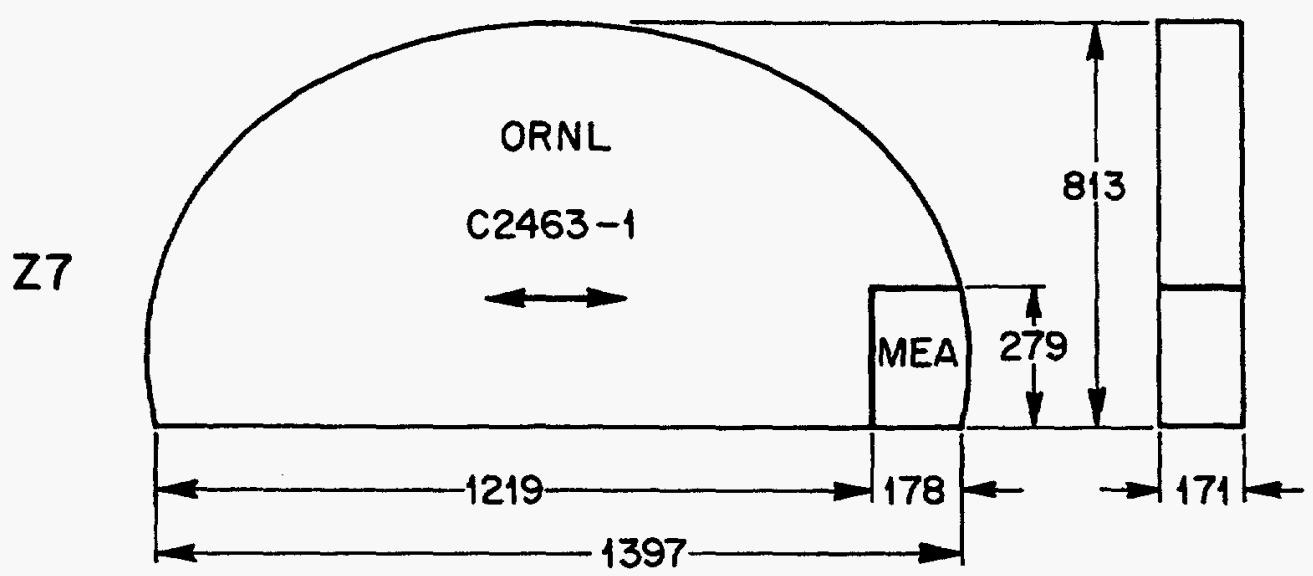

(d)

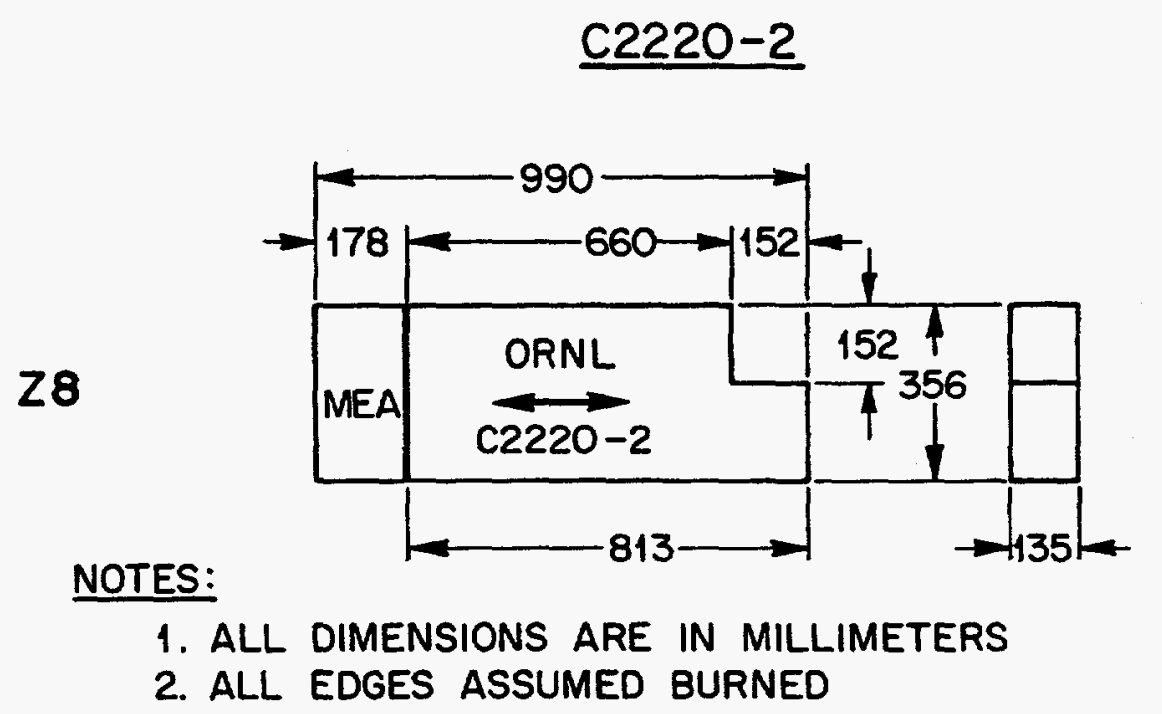

Figure 3. (Continued) 
Table 1. Fracture toughness of modified A 302 grade B - test matrix

\begin{tabular}{|c|c|c|c|c|c|}
\hline $\begin{array}{l}\text { Specimen } \\
\text { size }\end{array}$ & $\begin{array}{l}\text { Plate } \\
\text { code }\end{array}$ & $\begin{array}{c}\begin{array}{c}\text { Test } \\
\text { temperatures } \\
\left({ }^{\circ} \mathrm{F}\right)\end{array} \\
\end{array}$ & Orientation ${ }^{2}$ & Duplicates & $\begin{array}{l}\text { Number of } \\
\text { specimens } \\
\text { per plate }\end{array}$ \\
\hline $\begin{array}{l}1 / 2 \mathrm{~T} \mathrm{C}(\mathrm{T}) \\
\text { Total } \\
\end{array}$ & $\begin{array}{c}Z 1, Z 2 \\
Z 3 \\
Z 4 \\
Z 5 \\
Z 6 A \\
Z 6 B \\
Z 7 \\
Z 8\end{array}$ & $\begin{array}{l}180,400,550 \\
180,400,550 \\
180,400,550 \\
180,400,550 \\
180,400,550 \\
180,400,550 \\
180,400,550 \\
180,400,550\end{array}$ & $\begin{array}{l}\text { T-L, L-S } \\
\text { T-L, L-S } \\
\text { T-L, L-S } \\
\text { T-L, L-S } \\
\text { T-L, L-S } \\
\text { T-L, L-S } \\
\text { T-L, L-S } \\
\text { T-L, L-S }\end{array}$ & $\begin{array}{l}2 \\
2 \\
2 \\
2 \\
2 \\
2 \\
2 \\
2\end{array}$ & $\begin{array}{l}12 \\
12 \\
12 \\
12 \\
12 \\
12 \\
12 \\
12 \\
96 \\
\end{array}$ \\
\hline $\begin{array}{l}\text { 1T C (T) } \\
\text { Total }\end{array}$ & $\begin{array}{l}Z 2 \\
Z 3 \\
Z 5 \\
Z 6 A \\
Z 6 B \\
Z 7\end{array}$ & $\begin{array}{l}180,400,550 \\
180,550 \\
180,550 \\
180,550 \\
180,550 \\
180,400,550\end{array}$ & $\begin{array}{c}\text { T-L, L-S } \\
\text { T-L, L-S } \\
\text { L-T } \\
T-L, L-S \\
T-L, L-S \\
T-L, L-S\end{array}$ & $\begin{array}{l}2 \\
2 \\
2 \\
2 \\
2 \\
2\end{array}$ & $\begin{array}{r}12 \\
8 \\
4 \\
8 \\
8 \\
12 \\
52\end{array}$ \\
\hline $\begin{array}{l}2 \mathrm{~T} C(\mathrm{~T}) \\
\text { Total } \\
\end{array}$ & $\begin{array}{l}Z 1 \\
Z 2 \\
Z 3 \\
Z 4 \\
Z 5 \\
Z 6 A \\
Z 6 B \\
Z 7 \\
Z 8\end{array}$ & $\begin{array}{l}550 \\
180,400 \\
180,550 \\
180,550 \\
180,550 \\
180,550 \\
550 \\
180,400,550 \\
180,550\end{array}$ & $\begin{array}{c}\text { T-L, L-T } \\
\text { T-L, L-S } \\
\text { T-L, L-S, L-T } \\
\text { T-L, L-S } \\
\text { T-L, L-S } \\
\text { T-L, L-S } \\
\text { T-L, L-S, L-T } \\
\text { T-L, L-S, L-T } \\
\text { T-L, L-S }\end{array}$ & $\begin{array}{l}2 \\
2 \\
2 \\
2 \\
2 \\
2 \\
2 \\
2 \\
2 \\
2\end{array}$ & $\begin{array}{r}4 \\
8 \\
8 \\
8 \\
8 \\
8 \\
6 \\
16 \\
8 \\
74 \\
\end{array}$ \\
\hline $4 \mathrm{~T} \mathrm{C}(\mathrm{T})$ & $\begin{array}{l}Z 2 \\
Z 3 \\
Z 4 \\
Z 7 \\
Z 8\end{array}$ & $\begin{array}{l}180,550 \\
180 \\
180 \\
180,550 \\
180\end{array}$ & $\begin{array}{c}T-L \\
T-L \\
T-L \\
T-L, L-T \\
T-L\end{array}$ & $\begin{array}{l}2 \\
1 \\
1 \\
3 \\
1\end{array}$ & $\begin{array}{r}2 \\
1 \\
1 \\
6 \\
1 \\
11 \\
\end{array}$ \\
\hline Charpy & $\begin{array}{l}21 \\
23 \\
24 \\
25 \\
26 \\
27 \\
Z 8\end{array}$ & $\begin{array}{l}b \\
b \\
b \\
b \\
b \\
b \\
b\end{array}$ & $\begin{array}{c}\text { T-L, L-S, L-T } \\
\text { T-L, L-S, L-T } \\
\text { T-L, L-S } \\
\text { T-L, L-S, L-T } \\
\text { T-L, L-S, L-T } \\
\text { T-L, L-S, L-T } \\
\text { T-L, L-S, L-T }\end{array}$ & $\begin{array}{l}14 \\
15 \\
14 \\
14 \\
15 \\
15 \\
14\end{array}$ & $\begin{array}{r}42 \\
45 \\
28 \\
42 \\
45 \\
45 \\
42 \\
289\end{array}$ \\
\hline
\end{tabular}

4T compact specimens, because of the limited amount of material available. The sampling plan was designed to maximize the information production out of the available materials. Material baseline characterization on all materials included chemical compositions, CVN curves, drop-weight nil-ductility transition (NDT), and tensile tests in the three principal orientations. 



\section{Material Characterization}

The chemical composition of each heat was determined by ABB Combustion Engineering of Chattanooga, Tennessee. The results are presented in Table 2. Plate Z6B had sulfur comparable to the MEA plate, and the vanadium in all heats was about one-sixth of that in the MEA plate. Material chemistry does not appear to be a significant variable in this experiment.

Table 3 has tensile properties that cover the range of orientations and test temperatures of relevance to the $J-R$ curve determinations. The symbols $T, L$, and $S_{+}$represent transverse, longitudinal, and short transverse orientations, respectively. Note that there is a slight reversal in strength loss with increasing test temperature between 400 and $550^{\circ} \mathrm{F}\left(204\right.$ to $\left.288^{\circ} \mathrm{C}\right)$. Such behavior could be indicative of dynamic strain aging. Elongation measured in the short transverse orientation indicates that there might be a crack path of low toughness that develops at $550^{\circ} \mathrm{F}\left(288^{\circ} \mathrm{C}\right)$, one that has not been investigated in this study. The suggestion here is that the S-L or S-T crack propagation directions could respond differently. On the other hand, neither of these two crack plane orientations is likely to be of relevance in vessel analysis problems.

Tables 4 and 5 contain data for transition temperature characterization. Table 4 gives the type of CVN data that are used for the establishment of reference temperature, $\mathrm{RT}_{\mathrm{NDT}}$ (ref. 4), in the American Society of Mechanical Engineers (ASME) Boiler and Pressure Vessel Code. Drop-weight NDT values are determined, and the CVN property requirements at NDT $+60^{\circ} \mathrm{F}\left(+33^{\circ} \mathrm{C}\right)$ are evaluated, namely CVN energy $>50 \mathrm{ft}-\mathrm{lb}(68 \mathrm{~J})$ and lateral expansion $>0.035 \mathrm{in}$. $(0.9 \mathrm{~mm})$. If the CVN criteria are met, then $\mathrm{RT}_{\mathrm{NDT}}=\mathrm{NDT}$. Failure to meet these two criteria at $\mathrm{NDT}+60^{\circ} \mathrm{F}$ requires the use of Charpy transition curves to establish $R T_{\text {NDT }}$. Plates coded $Z 6 B$ and $Z 8$ had failed the criteria, and the additional conservatism imposed by the alternate code method can be noted. Table 5 gives additional Charpy information in the form of temperatures of 30 and $50 \mathrm{ft}-\mathrm{lb}, 50 \%$ shear, and the Charpy curve USEs. Of the three orientations, the T-L test direction is the one of lowest toughness in every case. The $L-T$ and L-S orientations show better toughness and at times have about the same USE.

Because the material chemical compositions are so close, the differences in toughness observed here are most likely due to differences in the slabbing, cross-rolling, and heat-treatment practices. 
Table 2. Modified A $\mathbf{3 0 2}$ grade $B$ test materials, heat code, and chemistries

\begin{tabular}{|c|c|c|c|c|c|c|c|c|c|c|c|c|c|c|}
\hline \multirow{2}{*}{$\begin{array}{l}\text { Plate } \\
\text { code }\end{array}$} & \multirow{2}{*}{ Heat } & \multicolumn{13}{|c|}{$\begin{array}{c}\text { Composition } \\
\text { (wt \%) }\end{array}$} \\
\hline & & C & $\mathbf{M n}$ & $\mathbf{P}$ & $\mathbf{s}$ & Si & $\mathrm{Ni}$ & $\mathrm{Cr}$ & Mo & v & $\mathbf{N b}$ & Co & $\mathrm{Cu}$ & Al \\
\hline$Z 1, Z 2$ & B3990-2 & 0.18 & 1.35 & 0.007 & 0.011 & 0.23 & 0.47 & 0.11 & 0.44 & 0.003 & 0.004 & 0.011 & 0.17 & 0.03 \\
\hline & $A 0237-1^{a}$ & 0.24 & 1.47 & 0.008 & 0.014 & 0.25 & 0.52 & 0.11 & 0.50 & 0.002 & 0.003 & 0.011 & 0.11 & 0.026 \\
\hline & $C 1290-2^{a}$ & 0.22 & 1.42 & 0.006 & 0.013 & 0.24 & 0.50 & 0.11 & 0.48 & 0.002 & 0.003 & 0.011 & 0.10 & 0.027 \\
\hline $\mathrm{Z5}$ & P2130-2 & 0.17 & 1.16 & 0.013 & 0.016 & 0.17 & 0.60 & 0.10 & 0.50 & 0.002 & 0.004 & 0.012 & 0.16 & 0.012 \\
\hline$Z 6 A$ & C1079-1 & 0.17 & 1.35 & 0.006 & 0.013 & 0.23 & 0.49 & 0.10 & 0.45 & 0.002 & 0.003 & 0.012 & 0.18 & 0.028 \\
\hline Z6B & B5013-2 & 0.22 & 1.39 & 0.009 & 0.023 & 0.20 & 0.51 & 0.15 & 0.48 & 0.002 & 0.003 & 0.017 & 0.21 & 0.035 \\
\hline 27 & C2463-1 & 0.26 & 1.47 & 0.008 & 0.014 & 0.15 & 0.53 & 0.09 & 0.52 & 0.002 & 0.003 & 0.011 & 0.16 & 0.017 \\
\hline $\mathbf{Z 8}$ & C2220-2 & 0.27 & 1.49 & 0.006 & 0.015 & 0.23 & 0.68 & 0.12 & 0.45 & 0.002 & 0.003 & 0.012 & 0.16 & 0.016 \\
\hline
\end{tabular}


Table 3. Tensile properties for seven heats of modified A 302 grade B (Z1-Z7) and one vintage heat of A 533 grade B material (Z8)

\begin{tabular}{|c|c|c|c|c|c|c|c|c|c|c|}
\hline \multirow{2}{*}{$\begin{array}{l}\text { Plate } \\
\text { code }\end{array}$} & \multirow{2}{*}{$\begin{array}{c}\text { Test } \\
\text { temperature } \\
\left({ }^{\circ} \mathrm{F}\right)\end{array}$} & \multicolumn{3}{|c|}{$\begin{array}{l}\text { Yield strength" } \\
\text { (ksi) }\end{array}$} & \multicolumn{3}{|c|}{$\begin{array}{l}\text { Ultimate strength } \\
\text { (ksi) }\end{array}$} & \multicolumn{3}{|c|}{$\begin{array}{c}\text { Elongation } \\
(\%)\end{array}$} \\
\hline & & $\mathbf{T}$ & L & $S_{T}$ & $\mathbf{T}$ & $\mathbf{L}$ & $\mathbf{S}_{T}$ & $\mathbf{T}$ & $\mathbf{L}$ & $S_{T}$ \\
\hline $\mathrm{Z1}$ & $\begin{array}{r}75 \\
180 \\
400 \\
550 \\
\end{array}$ & $\begin{array}{l}65.2 \\
62.4 \\
57.0 \\
60.3\end{array}$ & $\begin{array}{l}64.0 \\
64.2 \\
58.8 \\
60.1\end{array}$ & $\begin{array}{l}64.2 \\
58.1 \\
57.7 \\
58.9\end{array}$ & $\begin{array}{l}84.5 \\
80.7 \\
79.3 \\
84.8\end{array}$ & $\begin{array}{l}84.7 \\
80.8 \\
79.7 \\
84.0\end{array}$ & $\begin{array}{l}80.1 \\
79.0 \\
72.2 \\
57.0 \\
\end{array}$ & $\begin{array}{l}25.5 \\
22.5 \\
21.0 \\
24.5 \\
\end{array}$ & $\begin{array}{l}25.0 \\
23.0 \\
21.5 \\
24.0 \\
\end{array}$ & $\begin{array}{c}13.5 \\
17,7^{b} \\
15,8^{\circ} \\
8\end{array}$ \\
\hline 23 & $\begin{array}{r}75 \\
180 \\
400 \\
550\end{array}$ & $\begin{array}{l}67.9 \\
64.8 \\
59.8 \\
61.7\end{array}$ & $\begin{array}{l}67.8 \\
64.4 \\
59.5 \\
61.0\end{array}$ & $\begin{array}{l}67.0 \\
64.0 \\
52.9 \\
62.2\end{array}$ & $\begin{array}{l}89.7 \\
85.2 \\
84.9 \\
88.2\end{array}$ & $\begin{array}{l}90.0 \\
85.2 \\
84.2 \\
89.0\end{array}$ & $\begin{array}{l}89.3 \\
84.7 \\
80.5 \\
88.3\end{array}$ & $\begin{array}{l}23.0 \\
21.5 \\
20.5 \\
25.0\end{array}$ & $\begin{array}{l}27.5 \\
23.5 \\
23.0 \\
25.5\end{array}$ & $\begin{array}{l}16.5 \\
15.0 \\
11.0 \\
15.0\end{array}$ \\
\hline 24 & $\begin{array}{r}75 \\
180 \\
400 \\
550\end{array}$ & $\begin{array}{l}67.8 \\
63.8 \\
60.3 \\
61.2\end{array}$ & $\begin{array}{c}68.1 \\
64.4 \\
c \\
61.0\end{array}$ & $\begin{array}{l}66.5 \\
63.5 \\
c \\
60.4\end{array}$ & $\begin{array}{l}89.2 \\
83.9 \\
83.3 \\
88.0\end{array}$ & $\begin{array}{c}89.8 \\
84.7 \\
c \\
87.9\end{array}$ & $\begin{array}{l}88.2 \\
83.6 \\
c \\
87.1\end{array}$ & $\begin{array}{l}25.0 \\
22.5 \\
22.0 \\
24.0\end{array}$ & $\begin{array}{c}24.5 \\
23.5 \\
c \\
26.0\end{array}$ & $\begin{array}{c}21.5 \\
20.5 \\
c \\
20.0\end{array}$ \\
\hline Z5 & $\begin{array}{r}75 \\
180 \\
400 \\
550 \\
\end{array}$ & $\begin{array}{l}58.1 \\
55.6 \\
53.8 \\
54.2\end{array}$ & & $\begin{array}{l}55.2 \\
52.8 \\
49.0 \\
49.6\end{array}$ & $\begin{array}{l}78.1 \\
74.4 \\
75.8 \\
79.8\end{array}$ & & $\begin{array}{l}72.0 \\
68.6 \\
68.5 \\
74.1\end{array}$ & $\begin{array}{l}24.5 \\
22.4 \\
21.5 \\
23.4\end{array}$ & & $\begin{array}{l}17.5 \\
13.5 \\
13.0 \\
11.5\end{array}$ \\
\hline$Z 6 A$ & $\begin{array}{r}75 \\
180 \\
400 \\
550 \\
\end{array}$ & $\begin{array}{l}61.8 \\
57.9 \\
55.0 \\
55.8\end{array}$ & $\begin{array}{l}63.7 \\
59.1 \\
55.6 \\
57.7\end{array}$ & $\begin{array}{l}57.6 \\
58.4 \\
55.7 \\
57.5\end{array}$ & $\begin{array}{l}81.0 \\
70.8 \\
76.2 \\
81.2\end{array}$ & $\begin{array}{l}82.4 \\
77.0 \\
75.8 \\
84.0\end{array}$ & $\begin{array}{l}62.5 \\
61.5 \\
74.9 \\
76.8\end{array}$ & $\begin{array}{l}28.5 \\
16.0 \\
21.0 \\
21.5\end{array}$ & $\begin{array}{l}28.0 \\
25.0 \\
23.5 \\
22.0\end{array}$ & $\begin{array}{r}7.5 \\
4.5 \\
12.5 \\
12.0\end{array}$ \\
\hline Z6B & $\begin{array}{r}75 \\
180 \\
400 \\
550 \\
\end{array}$ & $\begin{array}{l}64.9 \\
64.5 \\
59.3 \\
60.7\end{array}$ & $\begin{array}{l}68.8 \\
63.2 \\
59.8 \\
61.5\end{array}$ & $\begin{array}{l}62.6 \\
61.0 \\
57.5 \\
60.7\end{array}$ & $\begin{array}{l}86.3 \\
83.6 \\
83.7 \\
86.2\end{array}$ & $\begin{array}{l}89.1 \\
82.3 \\
83.4 \\
87.2\end{array}$ & $\begin{array}{l}77.9 \\
78.0 \\
69.0 \\
64.7\end{array}$ & $\begin{array}{l}24.5 \\
20.5 \\
20.5 \\
21.5\end{array}$ & $\begin{array}{l}28.0 \\
24.0 \\
22.5 \\
24.0\end{array}$ & $\begin{array}{r}9.5 \\
11.0 \\
4.5 \\
5.0\end{array}$ \\
\hline $\mathrm{Z7}$ & $\begin{array}{r}75 \\
180 \\
400 \\
550\end{array}$ & $\begin{array}{l}68.5 \\
65.5 \\
60.1 \\
62.3\end{array}$ & $\begin{array}{l}69.1 \\
66.7 \\
60.3 \\
63.6\end{array}$ & $\begin{array}{l}68.2 \\
66.7 \\
61.4 \\
62.3\end{array}$ & $\begin{array}{l}91.7 \\
86.7 \\
85.6 \\
90.0\end{array}$ & $\begin{array}{l}92.5 \\
87.7 \\
86.0 \\
91.3\end{array}$ & $\begin{array}{l}92.2 \\
87.7 \\
86.2 \\
90.6\end{array}$ & $\begin{array}{l}24.5 \\
22.5 \\
21.5 \\
23.4\end{array}$ & $\begin{array}{l}23.5 \\
23.5 \\
22.5 \\
26.0\end{array}$ & $\begin{array}{l}17.5 \\
17.0 \\
13.0 \\
11.5\end{array}$ \\
\hline Z8 & $\begin{array}{r}75 \\
180 \\
400 \\
550\end{array}$ & $\begin{array}{l}61.9 \\
59.8 \\
56.7 \\
58.0\end{array}$ & $\begin{array}{l}63.1 \\
59.2 \\
54.7 \\
57.4\end{array}$ & $\begin{array}{l}61.6 \\
58.4 \\
55.8 \\
54.6\end{array}$ & $\begin{array}{l}88.2 \\
83.4 \\
82.7 \\
88.2\end{array}$ & $\begin{array}{l}88.9 \\
82.5 \\
80.2 \\
87.2\end{array}$ & $\begin{array}{l}87.2 \\
81.5 \\
80.0 \\
84.6\end{array}$ & $\begin{array}{l}23.5 \\
22.5 \\
21.0 \\
22.5\end{array}$ & $\begin{array}{l}26.0 \\
24.0 \\
23.0 \\
26.5\end{array}$ & $\begin{array}{l}17.5 \\
17.0 \\
14.5 \\
11.5\end{array}$ \\
\hline
\end{tabular}


Table 4. Reference temperature determinations

\begin{tabular}{|c|c|c|c|c|c|c|c|c|}
\hline \multirow{3}{*}{$\begin{array}{l}\text { Plate } \\
\text { code }\end{array}$} & \multirow{2}{*}{\multicolumn{2}{|c|}{$\begin{array}{c}\text { NDT }^{\mathbf{a}} \\
\text { temperature }\end{array}$}} & \multicolumn{4}{|c|}{$\begin{array}{c}\text { Charpy properties at } \\
\text { NDT }^{2}+60^{\circ} \mathrm{F} \\
\text { T-L (transverse) orientation }\end{array}$} & \multirow{2}{*}{\multicolumn{2}{|c|}{$R T_{\text {NOT }}^{b}$}} \\
\hline & & & \multicolumn{2}{|c|}{ Energy } & \multicolumn{2}{|c|}{$\begin{array}{c}\text { Lateral } \\
\text { expansion }\end{array}$} & & \\
\hline & $\left({ }^{\circ} \mathrm{F}\right)$ & $\left({ }^{\circ} \mathrm{C}\right)$ & (ft-lb) & (J) & (in.) & $(\mathrm{mm})$ & $\left({ }^{\circ} \mathrm{F}\right)$ & $\left({ }^{\circ} \mathrm{C}\right)$ \\
\hline $\begin{array}{l}Z 1, Z 2 \\
Z 3 \\
Z 4 \\
Z 5 \\
Z 6 A \\
Z 6 B \\
Z 7 \\
Z 8\end{array}$ & $\begin{array}{r}-5 \\
-15 \\
-15 \\
-30 \\
-30 \\
-30 \\
-15 \\
-20\end{array}$ & $\begin{array}{l}-20 \\
-26 \\
-26 \\
-34 \\
-34 \\
-34 \\
-26 \\
-29\end{array}$ & $\begin{array}{l}93 \\
60 \\
64 \\
56 \\
65 \\
38 \\
64 \\
33\end{array}$ & $\begin{array}{r}127 \\
82 \\
88 \\
77 \\
89 \\
52 \\
88 \\
45\end{array}$ & $\begin{array}{l}0.063 \\
0.046 \\
0.058 \\
0.048 \\
0.052 \\
0.034 \\
0.056 \\
0.038\end{array}$ & $\begin{array}{l}1.6 \\
1.2 \\
1.5 \\
1.2 \\
1.3 \\
0.9 \\
1.4 \\
1.0\end{array}$ & $\begin{array}{r}-5 \\
-15 \\
-15 \\
-30 \\
-30 \\
13^{c} \\
-15 \\
24^{c}\end{array}$ & $\begin{array}{r}-20 \\
-26 \\
-26 \\
-34 \\
-34 \\
-10 \\
-26 \\
-4\end{array}$ \\
\hline
\end{tabular}


Table 5. Charpy V-notch values for material transition temperature and upper-shelf evaluation

\begin{tabular}{|c|c|c|c|c|c|c|c|c|c|c|c|}
\hline \multirow{3}{*}{$\begin{array}{l}\begin{array}{l}\text { Plate } \\
\text { code }\end{array} \\
\mathrm{Z1}, \mathrm{Z2}\end{array}$} & \multirow{3}{*}{$\begin{array}{c}\text { Orientation" } \\
\text { T-L } \\
\text { L-T } \\
\text { L-S }\end{array}$} & \multicolumn{6}{|c|}{$\begin{array}{l}\text { Temperature, } \\
{ }^{\circ} \mathrm{F}\left({ }^{\circ} \mathrm{C}\right)\end{array}$} & \multicolumn{2}{|c|}{$R T_{\text {NDT }}{ }^{b}$} & \multicolumn{2}{|c|}{$\begin{array}{l}\text { Upper-shelf } \\
\text { energy }\end{array}$} \\
\hline & & \multicolumn{2}{|c|}{$30 \mathrm{ft}-1 \mathrm{~b}$} & \multicolumn{2}{|c|}{$50 \mathrm{ft}-1 \mathrm{~b}$} & \multicolumn{2}{|c|}{$50 \%$ shear } & \multirow{2}{*}{$\frac{\left({ }^{\circ} \mathbf{F}\right)}{-5}$} & \multirow{2}{*}{$\frac{\left({ }^{\circ} \mathrm{C}\right)}{-20}$} & \multirow{2}{*}{$\begin{array}{l}(\mathrm{ft}-\mathrm{lb}) \\
126 \\
160 \\
171\end{array}$} & \multirow{2}{*}{$\begin{array}{l}\text { (J) } \\
173 \\
219 \\
234\end{array}$} \\
\hline & & $\begin{array}{l}-33 \\
-45 \\
-19\end{array}$ & $\begin{array}{l}(-36) \\
(-43) \\
(-28)\end{array}$ & $\begin{array}{r}-4 \\
-20 \\
8\end{array}$ & $\begin{array}{l}(-20) \\
(-29) \\
(-13)\end{array}$ & & $\begin{array}{r}(-4) \\
(-12) \\
(-1)\end{array}$ & & & & \\
\hline $\mathbf{Z 3}$ & $\begin{array}{l}T-L \\
L-T \\
L-S\end{array}$ & $\begin{array}{r}0 \\
-37 \\
-16\end{array}$ & $\begin{array}{l}(-18) \\
(-38) \\
(-27)\end{array}$ & $\begin{array}{l}33 \\
-9 \\
21\end{array}$ & $\begin{array}{r}(0) \\
(-23) \\
(-6)\end{array}$ & $\begin{array}{l}35 \\
25 \\
55\end{array}$ & $\begin{array}{r}(2) \\
(-4) \\
(13)\end{array}$ & -15 & -26 & $\begin{array}{r}85 \\
129 \\
127\end{array}$ & $\begin{array}{l}116 \\
177 \\
174\end{array}$ \\
\hline Z4 & $\begin{array}{l}T-L \\
L-S\end{array}$ & $\begin{array}{l}-38 \\
-56\end{array}$ & $\begin{array}{l}(-39) \\
(-49)\end{array}$ & $\begin{array}{r}11 \\
-17\end{array}$ & $\begin{array}{l}(-12) \\
(-27)\end{array}$ & & $\begin{array}{r}(-1) \\
(2)\end{array}$ & -15 & -26 & $\begin{array}{l}111 \\
130\end{array}$ & $\begin{array}{l}152 \\
178\end{array}$ \\
\hline 25 & $\begin{array}{l}T-L \\
L-T \\
L-S\end{array}$ & $\begin{array}{l}-19 \\
-30 \\
-59\end{array}$ & $\begin{array}{l}(-28) \\
(-34) \\
(-50)\end{array}$ & $\begin{array}{r}18 \\
-2 \\
-41\end{array}$ & $\begin{array}{r}(-8) \\
(-19) \\
(-40)\end{array}$ & & $\begin{array}{r}(-7) \\
(-4) \\
(-23)\end{array}$ & -30 & -34 & $\begin{array}{r}95 \\
153 \\
171\end{array}$ & $\begin{array}{l}130 \\
209 \\
234\end{array}$ \\
\hline $26 A$ & $\begin{array}{l}\text { T-L } \\
\text { L-T } \\
\text { L-S }\end{array}$ & $\begin{array}{l}-17 \\
-28 \\
-41\end{array}$ & $\begin{array}{l}(-27) \\
(-33) \\
(-40)\end{array}$ & $\begin{array}{r}11 \\
-2 \\
-10\end{array}$ & $\begin{array}{l}(-12) \\
(-19) \\
(-23)\end{array}$ & & $\begin{array}{c}(4.4) \\
(-4) \\
(2)\end{array}$ & -30 & -34 & $\begin{array}{l}113 \\
129 \\
130\end{array}$ & $\begin{array}{l}155 \\
177 \\
178\end{array}$ \\
\hline Z6B & $\begin{array}{l}T-L \\
L-T \\
L-S\end{array}$ & $\begin{array}{r}11 \\
-33 \\
-32\end{array}$ & $\begin{array}{l}(-12) \\
(-36) \\
(-36)\end{array}$ & $\begin{array}{l}60 \\
-1 \\
-5\end{array}$ & $\begin{array}{r}(16) \\
(-18) \\
(-20)\end{array}$ & $\begin{array}{r}40 \\
5 \\
10\end{array}$ & $\begin{array}{l}(4.4) \\
(-15) \\
(-12)\end{array}$ & 13 & -10 & $\begin{array}{r}64 \\
117 \\
114\end{array}$ & $\begin{array}{r}88 \\
160 \\
156\end{array}$ \\
\hline $\mathbf{Z 7}$ & $\begin{array}{l}T-L \\
L-T \\
L-S\end{array}$ & $\begin{array}{l}-21 \\
-45 \\
-50\end{array}$ & $\begin{array}{l}(-29) \\
(-43) \\
(-46)\end{array}$ & $\begin{array}{r}19 \\
-12 \\
-20\end{array}$ & $\begin{array}{r}(-7) \\
(-24) \\
(-29)\end{array}$ & $\begin{array}{r}5 \\
10 \\
0\end{array}$ & $\begin{array}{l}(-15) \\
(-12) \\
(-18)\end{array}$ & -15 & -26 & $\begin{array}{r}96 \\
126 \\
120\end{array}$ & $\begin{array}{l}131 \\
173 \\
164\end{array}$ \\
\hline $\mathbf{Z 8}$ & $\begin{array}{l}T-L \\
L-T \\
L-S\end{array}$ & $\begin{array}{r}27 \\
25 \\
-5\end{array}$ & $\begin{array}{r}(-3) \\
(-4) \\
(-20)\end{array}$ & $\begin{array}{l}72 \\
48 \\
29\end{array}$ & $\begin{array}{r}(22) \\
(9) \\
(-2)\end{array}$ & $\begin{array}{l}30 \\
65 \\
55\end{array}$ & $\begin{array}{l}(-1) \\
(18) \\
(13)\end{array}$ & 24 & -4.4 & $\begin{array}{r}96 \\
134 \\
154\end{array}$ & $\begin{array}{l}131 \\
184 \\
211\end{array}$ \\
\hline
\end{tabular}





\section{Fracture Mechanics Data}

The test matrix introduced in Table 1 contains about 230 compact specimens for J-R curve tests. The results of each test were carefully evaluated, and any data judged questionable or that might have had a possible orientation identification error will not be reported or used in the present data analysis. Such cases were few and, for the most part, were outside the realm of control of these investigators. Volume 2 will log every test conducted, and all resulting test data, so that both good and questionable data will be available for further use as needed.

Material crack growth resistance is defined by the development rate of $\mathrm{J}$-integral associated with slow-stable crack growth (J-R curves). In this project, crack growth was measured by unloading compliance, following the requirements set forth in American Society for Testing and Materials (ASTM) E 813-87, "Standard Test Method for $J_{l c}$, a Measure of Fracture Toughness," and E 1152-89, "Standard Test Method for Determining J-R Curves."

$J-R$ curve plots are difficult to compare quantitatively, and it is more convenient to work with single-value parameters that are derived from the $\mathrm{J}-\mathrm{R}$ curve. One such parameter is the $\mathrm{J}_{\mathrm{k}}$ value, defined as $\mathrm{J}$-integral just after the onset of slow-stable crack growth [after 0.008 in. $(0.2 \mathrm{~mm})$ of ductile tearing]. ${ }^{5}$ The ASTM test practice for $J_{1 c}$ has undergone two revisions since it was first introduced in 1981, and comments on the implication of these revisions on precision and accuracy will be included here as supplementary information. A second single characterization parameter is tearing modulus, $\mathrm{T}$, a dimensionless quantity derived from the J-R curve slope. The third single-value parameter used is the J-integral after $0.1 \mathrm{in}$. $(2.5 \mathrm{~mm})$ of slow-stable crack growth, $J_{0.1}$.

The first-mentioned parameter, $J_{l e}$, has been a moving target, undergoing revisions in 1989 and 1995 . $J_{k}$ was calculated by all three methods, and the 1989 version was chosen as the best of the two new methods for developing material comparisons here. The rationale will be discussed in Sect. 5 of this report. The T-parameter and $J_{0.1} \mathrm{~J}-\mathrm{R}$ curve growth point values are the two material property parameters used in the ASME Code, Sect. XI, Articles K-4220 and K-4331 (ref. 6). It is interesting that the ASTM test methods give no guidance as to how these two design-related parameters are determined. This requires that users of T-modulus and $J_{0.1}$ values must develop their own definitions. Accordingly, for the purposes of this report, the $J_{0.1}$ value will specifically be the $J$ value after 0.1 in. of slow-stable crack growth that includes the blunting line contribution to $\Delta a$ (see Figure 4). The blunting line contribution is calculated using the following equation:

$$
\Delta \mathrm{a}=\mathrm{J}_{\mathrm{Ic}} /\left(\sigma_{\mathrm{ys}}+\sigma_{\mathrm{UTS}}\right)
$$

The T-modulus parameter is defined here as a nondimensional representation of the average J-R curve slope from the $\mathrm{J}-\Delta \mathrm{a}$ data that are developed between the two dashed exclusion lines shown. The dashed secant line shown in Figure 4 that extends between the two J-R curve intersection points with the exclusion lines is always close to the average slope determined if a set of tangents were taken along the J-R curve and an average value obtained. Here the secant slope, $(\mathrm{d} J / \mathrm{da})_{3}$, is used in the following expression:

$$
T_{\text {avg }}=\frac{E}{\sigma_{f}^{2}}\left(\frac{d J}{d a}\right)_{s} .
$$

The value, $\sigma_{f}$, is the denominator in Equation (1) divided by two.

A full J-R curve data plot with extensive growth can be well modeled by an equation of the following form:

$$
J=A(\Delta a)^{B} \exp (C / \sqrt{\Delta a})
$$




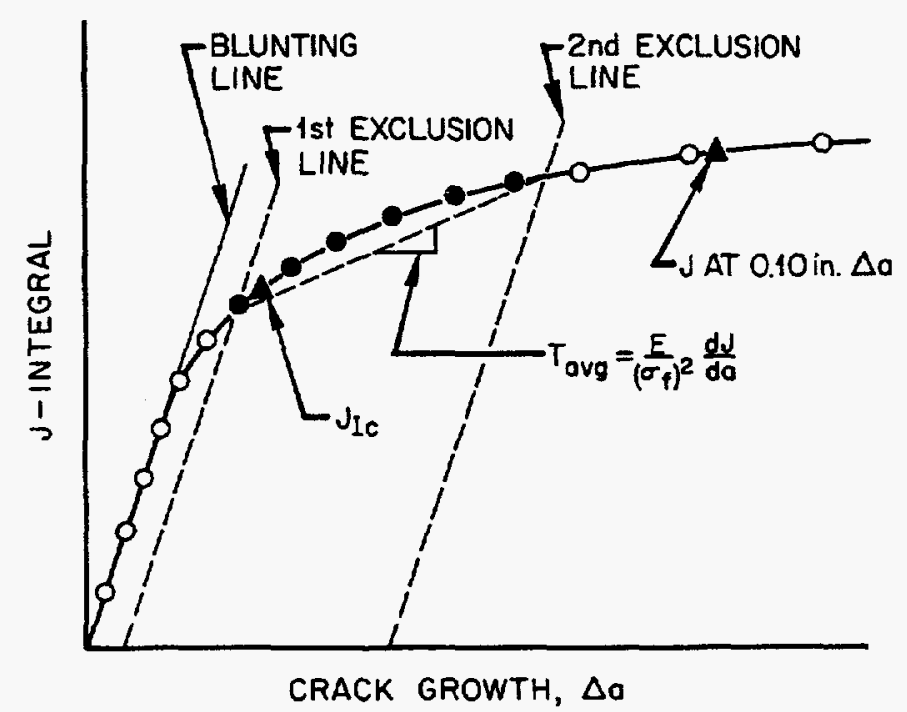

Figure 4. Schematic of J-R curve plot and measurements taken. Filled data points represent the points used in the power law curve fit.

Equation (3) had been introduced by Eason et al. in multivariable modeling of J-R curves for $A 533$ grade $B$ base metal and various reactor pressure vessel weld metals. ${ }^{7}$ In their work they correlate nonfracture mechanics information, such as upper-shelf CVN energy or copper/fluence models, to derive the constants $A, B$, and $C$ for Equation (3). In this study, Equation (3) is only used to best fit experimentally developed J-R curves, i.e., ones that have been chosen to best represent the materials and test conditions cited in the text matrix. 


\section{J-R Curve Characteristics of Seven Heats of Modified A 302 Grade B Steel}

\subsection{Size Effects}

Table 6 lists the average $T, J_{0.1}$, and $J_{l c}$ values from duplicate specimens (single specimens in a few cases) for each specimen size at $180^{\circ} \mathrm{F}\left(82^{\circ} \mathrm{C}\right)$. The same parameters were calculated from the J-R curve plots presented in the MEA report on their plate, V50, of $A 302$ grade $B$ steel (ref. 2). In that case, only the T-L orientation and one test temperature $\left[180^{\circ} \mathrm{F}\left(82^{\circ} \mathrm{C}\right)\right]$ had been used to evaluate specimen size effects. Clearly, the MEA result of decreased crack growth resistance with increased specimen size was not reproduced in the modified A 302 grade $B$ materials of this study. In fact, only one heat came close to the low toughness of the MEA V50 plate; this was Z6B and no inversion of size effect between $1 / 2 T$ to $2 T$ size was shown. Unfortunately, there was not sufficient material of Z6B to make a 4T compact specimen that could have completely settled this size effect issue. On the other hand, plate $Z 7$ was chosen to represent the J-R curve toughness level for the majority of the modified A 302 grade $B$ heats, and the typical J-R curve result is compared to the MEA heat V50 in Figure 5.

A peculiar size effect that can be observed in Figure 5 and in the Table 6 data, however, is that $2 T$ specimens tend to show a slight increased toughness bias in J-R curve performance. One could reasonably doubt this observation if the evidence were available only in terms of $J_{1 c}$ values where high preciseness of compliance-indicated crack growth is needed to determine the minuscule $0.008 \mathrm{in} .(0.2 \mathrm{~mm})$ of ductile crack extension. However, the $J_{0.1}$ values are not subject to the high precision need, and the increased toughness behavior in $2 \mathrm{~T}$ specimens is seen here as well. This $J-R$ curve enhancement is most likely a constraint-based phenomenon, but whether the observation represents increased or decreased constraint remains unproven as of now.

\subsection{Orientation Effects}

Table 7 shows the crack-plane orientation effect on fracture toughness. The three directions are transverse (T-L), longitudinal (L-T), and short transverse (L-S). ${ }^{3}$ Only one test temperature was needed for this evaluation, and because size effects were not particularly overhweiming, the values of $T, J_{0.1}$, and $J_{1 c}$ have been averaged over all specimen sizes. Additional information is presented in the form of Charpy USEs, and the last three columns of Table 7 list the coefficients needed in Equation (3) to generate the one individual specimen J-R curve that is most representative for the given plate and orientation. The choice of $J-R$ curve was made considering the closeness of fit to the averaged T-modulus, $J_{0.1}$, and $J_{l c}$ values listed. One $J-R$ curve is given for each orientation. The codes for the specimens selected are listed under the column entitled "Fitting curves," and the curves, plus the J- $\Delta \mathrm{a}$ data used, are shown in Appendix A, Figures A1 through A21.

It is not possible to make an all-encompassing statement about directionality effects on toughness for modified A 302 grade B pressure vessel steels because the directionality characteristics are created in the slabbing and cross-rolling practices used by the steel plant. In this experiment, each heat had been marked for the principal rolling direction, and both the Charpy data and J-R curve tests tended to verify these markings. The T-L orientation consistently showed the lowest toughness of the three orientations sampled. Heat code Z3 seemed to give an inconsistent pattern. This same heat is the one which had the most specimens that were subjected to orientation questions needing verification by metallographic methods.

\subsection{Test Temperature Effects}

Table 8 shows the effect of test temperature on the J-R curve. Again, as with Table 7, the values of $T, J_{0.1}$, and $J_{1 c}$ are averaged over duplicate tests and over all available specimen sizes. The comparison in this case is limited to one orientation, T-L. Representative $J-R$ curves that come the closest to satisfying the averaged $T$ slope, $J_{0.1}$, and $J_{\mathrm{Ic}}$ values can be reproduced from the coefficients listed. These curves, and specific J-R curve data used, are shown in Appendix $B$.

The trend of decreased crack-initiation toughness, $J_{l c}$, and J-R curve crack growth resistance with elevated test temperature is typical for all grades of reactor vessel steels. However, something that may have gone unnoticed in other experiments, which has been apparent in these tests, is that crack pop-in events had developed in specimens 
Table 6. Evaluation of specimen size on J-R curves of modified A 302 grade B steel [averaged values; T-L (transverse) orientation and $180^{\circ} \mathrm{F}\left(82^{\circ} \mathrm{C}\right)$; all specimens $20 \%$ side grooved]

\begin{tabular}{|c|c|c|c|c|}
\hline $\begin{array}{l}\text { Plate } \\
\text { code }\end{array}$ & $\begin{array}{l}\text { Size } \\
\text { (T) }\end{array}$ & $\mathbf{T}^{\mathbf{a}}$ & $\begin{array}{c}J_{0.1}{ }^{b} \\
\left(\text { in.-lb/in. }{ }^{2}\right)\end{array}$ & $\begin{array}{c}J_{l_{c}{ }^{c}} \\
\left.\text { (in.-lb/in. }{ }^{2}\right)\end{array}$ \\
\hline $\mathrm{Z1}, \mathrm{Z2}$ & $\begin{array}{l}1 / 2 \\
1 \\
2 \\
4\end{array}$ & $\begin{array}{r}115 \\
130 \\
120 \\
75\end{array}$ & $\begin{array}{l}3200 \\
3410 \\
4030 \\
2990\end{array}$ & $\begin{array}{l}1330 \\
1360 \\
2050 \\
1590\end{array}$ \\
\hline 23 & $\begin{array}{l}1 / 2 \\
1 \\
2 \\
4\end{array}$ & $\begin{array}{r}85 \\
80 \\
115 \\
95\end{array}$ & $\begin{array}{l}2615 \\
2515 \\
3055 \\
2755\end{array}$ & $\begin{array}{l}1230 \\
1175 \\
1095 \\
1310\end{array}$ \\
\hline $\mathbf{Z 4}$ & $\begin{array}{l}1 / 2 \\
2 \\
4\end{array}$ & $\begin{array}{r}105 \\
95 \\
95\end{array}$ & $\begin{array}{l}3050 \\
3200 \\
2840\end{array}$ & $\begin{array}{l}1360 \\
1600 \\
1200\end{array}$ \\
\hline $\mathbf{Z 5}$ & $\begin{array}{l}1 / 2 \\
2\end{array}$ & $\begin{array}{l}55 \\
50\end{array}$ & $\begin{array}{l}1655 \\
1825\end{array}$ & $\begin{array}{r}815 \\
1080\end{array}$ \\
\hline$Z 6 A$ & $\begin{array}{l}1 / 2 \\
1 \\
2\end{array}$ & $\begin{array}{l}80 \\
85 \\
55\end{array}$ & $\begin{array}{l}2370 \\
2535 \\
2070\end{array}$ & $\begin{array}{r}905 \\
1065 \\
1075\end{array}$ \\
\hline Z6B & $\begin{array}{l}1 / 2 \\
1 \\
2\end{array}$ & $\begin{array}{l}50 \\
45 \\
35\end{array}$ & $\begin{array}{l}1470 \\
1505 \\
1430\end{array}$ & $\begin{array}{l}715 \\
785 \\
930\end{array}$ \\
\hline $\mathbf{Z 7}$ & $\begin{array}{l}1 / 2 \\
1 \\
2 \\
4\end{array}$ & $\begin{array}{r}80 \\
95 \\
100 \\
125\end{array}$ & $\begin{array}{l}2600 \\
2995 \\
3325 \\
3120\end{array}$ & $\begin{array}{l}1290 \\
1210 \\
1685 \\
1270\end{array}$ \\
\hline $\mathbf{Z 8}$ & $\begin{array}{l}1 / 2 \\
2 \\
4\end{array}$ & $\begin{array}{l}95 \\
85 \\
70\end{array}$ & $\begin{array}{l}2770 \\
2525 \\
2800\end{array}$ & $\begin{array}{l}1290 \\
1160 \\
1740\end{array}$ \\
\hline \multicolumn{5}{|c|}{ MEA ${ }^{d}$ report on plate V50 } \\
\hline $\begin{array}{l}\text { p. } 58 \\
\text { p. } 61 \\
\text { p. } 66 \\
\text { p. } 70 \\
\text { p. } 74\end{array}$ & $\begin{array}{l}1 / 2 \\
1 \\
2 \\
4 \\
6\end{array}$ & $\begin{array}{l}36 \\
36 \\
20.4 \\
14.3 \\
19.4\end{array}$ & $\begin{array}{r}1293 \\
1129 \\
863 \\
704 \\
686\end{array}$ & $\begin{array}{l}600 \\
659 \\
653 \\
543 \\
500\end{array}$ \\
\hline $\begin{array}{l}{ }^{0} \mathrm{~T}=\mathrm{E} / \sigma_{\mathrm{i}} \\
{ }^{\mathrm{J}} \mathrm{J} \text { at } 0.1 \\
{ }_{\mathrm{J}} \mathrm{d} \text { after } 0 \\
{ }^{d} \mathrm{MEA}=1\end{array}$ & s. & ble & rowth. & 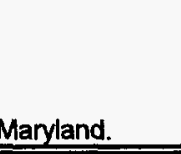 \\
\hline
\end{tabular}




\section{ORNL-DWG96-3458}

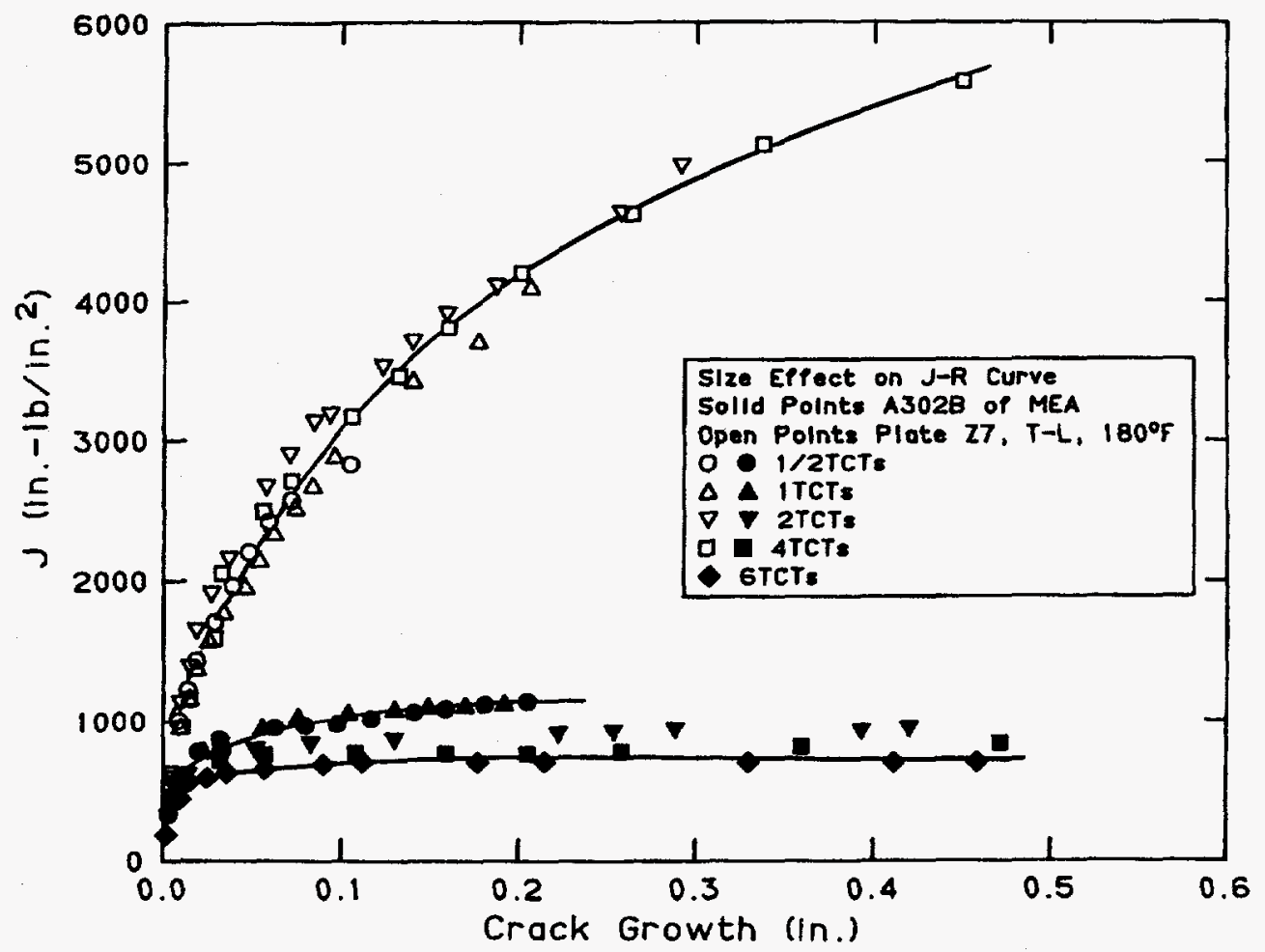

Figure 5. J-R curves from compact specimens of varied size for the typical modified A 302 grade B steel versus the Materials Engineering Associates plate V50 of A 302 grade B steel. 
Table 7. Directionality of $J-R$ curve fracture toughness at 180 " $F$ (averaged values over all specimen sizes; all specimens $20 \%$ side grooved)

N

\begin{tabular}{|c|c|c|c|c|c|c|c|c|c|}
\hline \multirow{2}{*}{$\begin{array}{l}\text { Plate } \\
\text { code }\end{array}$} & \multirow{2}{*}{ Orientation ${ }^{2}$} & \multirow{2}{*}{$\begin{array}{l}\text { Charpy V-notch } \\
\text { upper-shelf energy } \\
\text { (ft-lb) }\end{array}$} & \multirow{2}{*}{$\begin{array}{c}T \\
\text { slope }\end{array}$} & \multirow{2}{*}{$\begin{array}{c}J_{0.1}{ }^{b} \\
\left(\text { in.//b/in. }{ }^{2}\right)\end{array}$} & \multirow{2}{*}{$\begin{array}{c}\mathbf{J}_{\mathrm{lc}}{ }^{b} \\
\left.\text { (in./lb/in. }{ }^{2}\right)\end{array}$} & \multirow{2}{*}{$\begin{array}{l}\text { Fitting } \\
\text { curves }\end{array}$} & \multicolumn{3}{|c|}{$J=A \cdot \Delta a^{B} \cdot \exp \left(C / \Delta a^{1 / 2}\right)$} \\
\hline & & & & & & & A & B & C \\
\hline $\mathrm{Z1}, \mathrm{Z2}$ & $\begin{array}{l}T-L \\
L-T \\
L-S\end{array}$ & $\begin{array}{l}126 \\
160 \\
171\end{array}$ & $\begin{array}{l}120 \\
130 \\
130 \\
\end{array}$ & $\begin{array}{l}3300 \\
3810 \\
3480 \\
\end{array}$ & $\begin{array}{l}1350 \\
1360 \\
1570 \\
\end{array}$ & $\begin{array}{l}\mathrm{Z2B2-1T} \\
\mathrm{Z2B16-1T} \\
\mathrm{Z2B2-1/2T}\end{array}$ & $\begin{array}{l}7.110 \\
9.513 \\
8.715\end{array}$ & $\begin{array}{l}0.287 \\
0.300 \\
0.259\end{array}$ & $\begin{array}{l}-0.0596 \\
-0.0761 \\
-0.0846\end{array}$ \\
\hline 23 & $\begin{array}{l}T-L \\
L-T \\
L-S\end{array}$ & $\begin{array}{r}85 \\
129 \\
127 \\
\end{array}$ & $\begin{array}{l}90 \\
70\end{array}$ & $\begin{array}{l}2730 \\
2000\end{array}$ & $\begin{array}{r}1200 \\
870 \\
\end{array}$ & $\begin{array}{l}Z 310-1 / 2 T \\
Z 34-1 / 2 T\end{array}$ & $\begin{array}{l}6.030 \\
5.633\end{array}$ & $\begin{array}{l}0.275 \\
0.435\end{array}$ & $\begin{array}{l}-0.0699 \\
-0.0014\end{array}$ \\
\hline Z4 & $\begin{array}{l}T-L \\
L-S\end{array}$ & $\begin{array}{l}111 \\
130\end{array}$ & $\begin{array}{l}100 \\
130\end{array}$ & $\begin{array}{l}3060 \\
4080\end{array}$ & $\begin{array}{l}1420 \\
1880\end{array}$ & $\begin{array}{l}Z 49-1 / 2 T \\
Z 47-2 T\end{array}$ & $\begin{array}{r}6.517 \\
13.333\end{array}$ & $\begin{array}{l}0.226 \\
0.446\end{array}$ & $\begin{array}{l}-0.0368 \\
-0.0246\end{array}$ \\
\hline Z5 & $\begin{array}{l}T-L \\
L-T \\
L-S\end{array}$ & $\begin{array}{r}95 \\
153 \\
171\end{array}$ & $\begin{array}{r}45 \\
90 \\
160\end{array}$ & $\begin{array}{l}1630 \\
2640 \\
3895\end{array}$ & $\begin{array}{l}1075 \\
1225 \\
1195\end{array}$ & $\begin{array}{l}Z 55-2 T \\
Z 51-1 T \\
Z 57-1 / 2 T\end{array}$ & $\begin{array}{r}3.043 \\
6.178 \\
15.718\end{array}$ & $\begin{array}{l}0.177 \\
0.318 \\
0.567\end{array}$ & $\begin{array}{l}-0.0329 \\
-0.0348 \\
-0.0199 \\
\end{array}$ \\
\hline $26 \mathrm{~A}$ & $\begin{array}{l}\text { T-L } \\
\text { L-T } \\
\text { L-S }\end{array}$ & $\begin{array}{l}113 \\
129 \\
130 \\
\end{array}$ & $\begin{array}{r}75 \\
115 \\
120 \\
\end{array}$ & $\begin{array}{l}2325 \\
3570 \\
3550 \\
\end{array}$ & $\begin{array}{l}1040 \\
1570 \\
1620 \\
\end{array}$ & $\begin{array}{l}Z 6 A 8-1 T \\
Z 6 A 1-1 T \\
\text { Z6A3-1/2T } \\
\end{array}$ & $\begin{array}{r}6.377 \\
12.299 \\
8.542\end{array}$ & $\begin{array}{l}0.410 \\
0.518 \\
0.267\end{array}$ & $\begin{array}{l}-0.0131 \\
-0.0114 \\
-0.0752\end{array}$ \\
\hline Z6B & $\begin{array}{l}T-L \\
\text { L-T } \\
\text { L-S }\end{array}$ & $\begin{array}{r}64 \\
117 \\
114 \\
\end{array}$ & $\begin{array}{l}45 \\
70 \\
85 \\
\end{array}$ & $\begin{array}{l}1470 \\
2360 \\
2730 \\
\end{array}$ & $\begin{array}{r}810 \\
1130 \\
1183 \\
\end{array}$ & $\begin{array}{l}Z 6 B 9-1 / 2 T \\
Z 6 B 1-1 T \\
Z 6 B 8-1 / 2 T \\
\end{array}$ & $\begin{array}{l}2.407 \\
5.193 \\
1.340\end{array}$ & $\begin{array}{l}0.100 \\
0.341 \\
0.079\end{array}$ & $\begin{array}{l}-0.0790 \\
-0.0140 \\
-0.0235\end{array}$ \\
\hline 27 & $\begin{array}{l}T-L \\
L-T \\
L-S\end{array}$ & $\begin{array}{r}96 \\
126 \\
120\end{array}$ & $\begin{array}{r}95 \\
140 \\
145\end{array}$ & $\begin{array}{l}3000 \\
4500 \\
4405\end{array}$ & $\begin{array}{l}1250 \\
2120 \\
2045\end{array}$ & $\begin{array}{l}Z 711-1 T \\
Z 78-1 T \\
Z 71-1 / 2 T\end{array}$ & $\begin{array}{r}8.742 \\
12.057 \\
10.637 \\
\end{array}$ & $\begin{array}{l}0.446 \\
0.383 \\
0.326 \\
\end{array}$ & $\begin{array}{l}-0.0279 \\
-0.0501 \\
-0.0780 \\
\end{array}$ \\
\hline 28 & $\begin{array}{l}T-L \\
L-T \\
L-S\end{array}$ & $\begin{array}{r}96 \\
134 \\
154\end{array}$ & $\begin{array}{r}85 \\
145\end{array}$ & $\begin{array}{l}2715 \\
4190\end{array}$ & $\begin{array}{l}1245 \\
1860\end{array}$ & $\begin{array}{l}Z 83-1 / 2 T \\
Z 816-1 / 2 T\end{array}$ & $\begin{array}{r}4.963 \\
12.594\end{array}$ & $\begin{array}{l}0.218 \\
0.378\end{array}$ & $\begin{array}{l}-0.0514 \\
-0.0566\end{array}$ \\
\hline
\end{tabular}


Table 8. Test temperature effect on $J-R$ curve [averaged values over all specimen sizes; $T-L$ (transverse) orientation; all specimens $20 \%$ side grooved]

\begin{tabular}{|c|c|c|c|c|c|c|c|c|}
\hline \multirow{2}{*}{$\begin{array}{l}\text { Plate } \\
\text { code }\end{array}$} & \multirow{2}{*}{$\begin{array}{c}\text { Temperature } \\
\left({ }^{\circ} \mathbf{F}\right)\end{array}$} & \multirow{2}{*}{$\begin{array}{c}T \\
\text { slope }\end{array}$} & \multirow{2}{*}{$\begin{array}{c}\mathrm{J}_{0.1}^{2} \\
\left.\text { (in./lb/in. }{ }^{2}\right)\end{array}$} & \multirow{2}{*}{$\begin{array}{c}J_{1 c} a \\
\left(\text { in./lb/in. }{ }^{2}\right)\end{array}$} & \multirow{2}{*}{$\begin{array}{l}\text { Fitting } \\
\text { curves }\end{array}$} & \multicolumn{3}{|c|}{$J=A \cdot \Delta a^{B} \cdot \exp \left(C / \Delta a^{1 / 2}\right)$} \\
\hline & & & & & & A & B & C \\
\hline $\mathrm{Z1,}, \mathrm{Z2}$ & $\begin{array}{l}180 \\
400 \\
550\end{array}$ & $\begin{array}{r}120 \\
70 \\
60\end{array}$ & $\begin{array}{l}3300 \\
2310 \\
2056\end{array}$ & $\begin{array}{r}1350 \\
1100 \\
885\end{array}$ & $\begin{array}{l}\text { Z2B2-1T } \\
\text { Z2B11- } \\
1 / 2 T \\
\text { Z2B07-1T }\end{array}$ & $\begin{array}{l}7.110 \\
6.022 \\
5.619\end{array}$ & $\begin{array}{l}0.287 \\
0.362 \\
0.421\end{array}$ & $\begin{array}{l}-0.0596 \\
-0.0223 \\
-0.0095\end{array}$ \\
\hline$Z 3$ & $\begin{array}{l}180 \\
400 \\
550\end{array}$ & $\begin{array}{l}90 \\
80 \\
45\end{array}$ & $\begin{array}{l}2730 \\
2130 \\
1635\end{array}$ & $\begin{array}{r}1200 \\
830 \\
830\end{array}$ & $\begin{array}{l}Z 310-1 / 2 T^{b} \\
Z 311-1 / 2 T \\
Z 39-1 / 2 T\end{array}$ & $\begin{array}{l}6.030 \\
5.713 \\
3.394 \\
\end{array}$ & $\begin{array}{l}0.275 \\
0.362 \\
0.282\end{array}$ & $\begin{array}{r}-0.0699 \\
-0.0329 \\
-0.0268\end{array}$ \\
\hline Z4 & $\begin{array}{l}180 \\
400 \\
550 \\
\end{array}$ & $\begin{array}{r}100 \\
80 \\
60 \\
\end{array}$ & $\begin{array}{l}3060 \\
1995 \\
1950\end{array}$ & $\begin{array}{r}1420 \\
1020 \\
975\end{array}$ & $\begin{array}{l}Z 49-1 / 2 T^{b} \\
Z 416-1 / 2 T \\
Z 42-2 T\end{array}$ & $\begin{array}{l}6.517 \\
4.858 \\
3.252 \\
\end{array}$ & $\begin{array}{l}0.226 \\
0.297 \\
0.204\end{array}$ & $\begin{array}{r}-0.0368 \\
-0.0318 \\
0.0311\end{array}$ \\
\hline Z5 & $\begin{array}{l}180 \\
400 \\
550 \\
\end{array}$ & $\begin{array}{l}45 \\
40 \\
35\end{array}$ & $\begin{array}{l}1630 \\
1475 \\
1135\end{array}$ & $\begin{array}{r}1075 \\
895 \\
585\end{array}$ & $\begin{array}{l}Z 55-2 T^{b} \\
Z 514-1 / 2 T \\
Z 512-1 / 2 T\end{array}$ & $\begin{array}{l}3.043 \\
2.335 \\
2.267 \\
\end{array}$ & $\begin{array}{l}0.177 \\
0.136 \\
0.233\end{array}$ & $\begin{array}{l}-0.0329 \\
-0.0600 \\
-0.0188\end{array}$ \\
\hline$Z 6 A$ & $\begin{array}{l}180 \\
400 \\
550\end{array}$ & $\begin{array}{l}75 \\
55 \\
50\end{array}$ & $\begin{array}{l}2325 \\
1685 \\
1595\end{array}$ & $\begin{array}{r}1040 \\
805 \\
825\end{array}$ & $\begin{array}{l}Z 6 A 8-1 T^{D} \\
Z 6 A 16- \\
1 / 2 T \\
Z 6 A 15- \\
1 / 2 T\end{array}$ & $\begin{array}{l}6.377 \\
4.490 \\
3.383\end{array}$ & $\begin{array}{l}0.410 \\
0.343 \\
0.303\end{array}$ & $\begin{array}{l}-0.0131 \\
-0.0216 \\
-0.0201\end{array}$ \\
\hline Z6B & $\begin{array}{l}180 \\
400 \\
550\end{array}$ & $\begin{array}{l}45 \\
32 \\
24\end{array}$ & $\begin{array}{r}1470 \\
1175 \\
885\end{array}$ & $\begin{array}{l}810 \\
665 \\
530\end{array}$ & $\begin{array}{l}\text { Z6B9-1/2T } \\
\text { Z6B11- } \\
1 / 2 T \\
\text { Z6B7-1T }\end{array}$ & $\begin{array}{l}2.407 \\
1.801 \\
1.301\end{array}$ & $\begin{array}{l}0.100 \\
0.150 \\
0.146\end{array}$ & $\begin{array}{l}-0.0790 \\
-0.0352 \\
-0.0113\end{array}$ \\
\hline $\mathrm{Z7}$ & $\begin{array}{l}180 \\
400 \\
550 \\
\end{array}$ & $\begin{array}{l}95 \\
60 \\
50\end{array}$ & $\begin{array}{l}2995 \\
1995 \\
1855 \\
\end{array}$ & $\begin{array}{r}1255 \\
995 \\
790 \\
\end{array}$ & 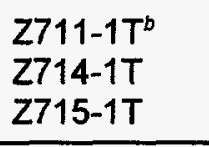 & $\begin{array}{l}8.742 \\
4.220 \\
3.487 \\
\end{array}$ & $\begin{array}{l}0.446 \\
0.340 \\
0.281 \\
\end{array}$ & $\begin{array}{r}-0.0279 \\
-0.0151 \\
-0.0140 \\
\end{array}$ \\
\hline Z8 & $\begin{array}{l}180 \\
400 \\
550\end{array}$ & $\begin{array}{l}85 \\
65 \\
50\end{array}$ & $\begin{array}{l}2715 \\
2095 \\
1685\end{array}$ & $\begin{array}{r}1250 \\
1070 \\
860\end{array}$ & $\begin{array}{l}Z 83-1 / 2 T^{b} \\
Z 88-1 / 2 T\end{array}$ & $\begin{array}{l}4.963 \\
3.889\end{array}$ & $\begin{array}{l}0.218 \\
0.393\end{array}$ & $\begin{array}{r}-0.0514 \\
0.0124\end{array}$ \\
\hline
\end{tabular}


tested at the upper-shelf temperature of $550^{\circ} \mathrm{F}\left(288^{\circ} \mathrm{C}\right)$. Usually, crack pop-in is associated with cleavage crack instability in the middle to just above the lower shelf of the transition temperature range. This upper-shelf pop-in behavior can be somewhat disguised in small specimens such as 1/2 and $1 T$ compact specimens and, hence, may be overlooked. Figures 6 through 8 represent the small specimen evidence, showing how obscure the pop-in behavior development can be between 180 and $550^{\circ} \mathrm{F}\left(82\right.$ and $\left.288^{\circ} \mathrm{C}\right)$. The evidence of crack instabilities in Figure 8 is weak enough to be disregarded. The contrast in load-displacement behavior observable in larger specimens is represented in Figures 9 and 10. Here the development of pop-ins is clear for the larger 2T specimen tested at $550^{\circ} \mathrm{F}\left(288^{\circ} \mathrm{C}\right)$. Each of the large displacement steps was caused by rapid crack propagation and arrest events. Such behavior was found to be chronic in heat $\mathrm{Z6B}$, developing in specimens of all orientations, but almost always at $550^{\circ} \mathrm{F}$. The other heats also showed pop-in behavior in tests at $550^{\circ} \mathrm{F}$, but generally of less frequency and, again, tending to favor large specimens of $T-L$ orientation. At first, dynamic strain aging was believed to be the cause of the pop-in behavior. On the other hand, the physical evidence seems to suggest otherwise. The pop-in phenomenon tended to favor material of lowest J-R curve slope and almost always occurred in the larger specimens that have the greatest elastic stored strain energy and greatest constraint. The grips of large specimens also have the most elastic stored strain energy. This information suggests, therefore, that these pop-ins are ductile crack instabilities, with the initiations being due to the crack-driving force exceeding the J-R curve resistance of the material. This phenomenon should be evaluated further to confirm the postulated cause. Crack instability from any cause may be sustained in structures that have a susbstantial reserve of elastic stored strain energy.

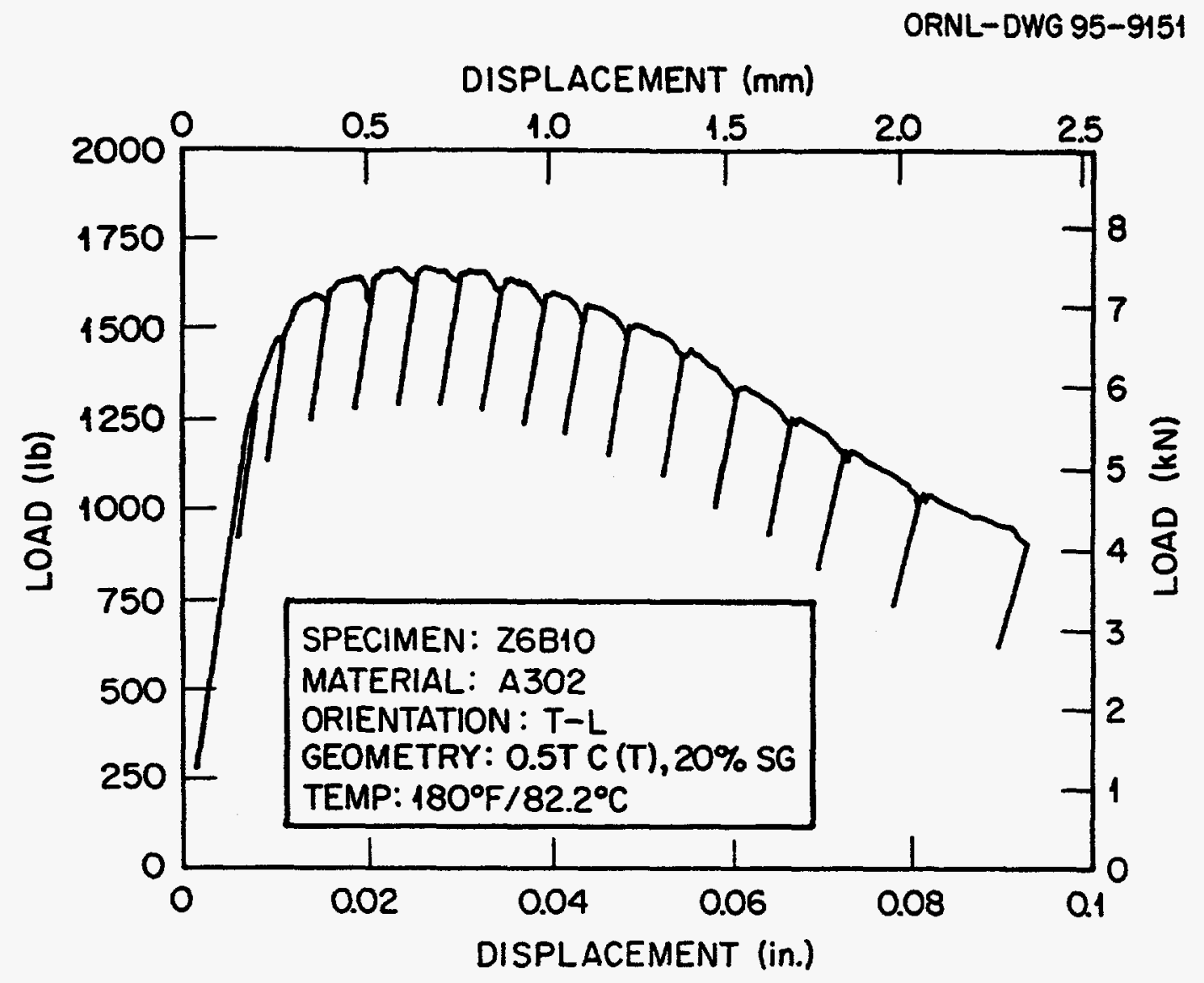

Figure 6. Load-displacement record of 1/2T compact specimen tested at $180^{\circ} \mathrm{F}\left(82^{\circ} \mathrm{C}\right)$, showing no evidence of pop-in. 


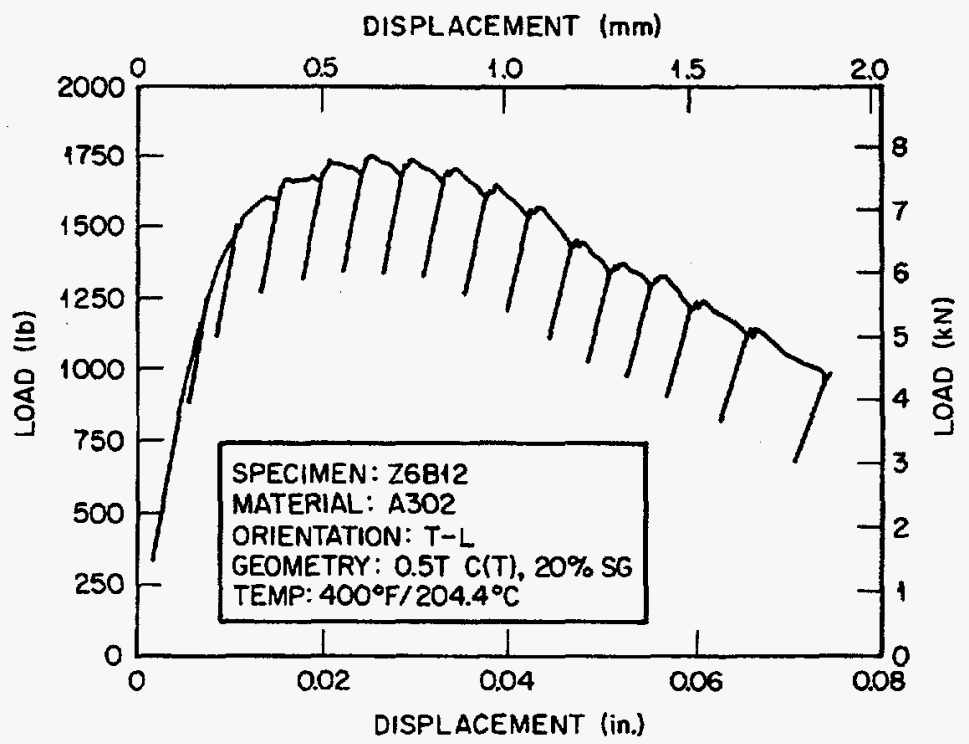

Figure 7. Load-displacement record of 1/2T compact specimen tested at $400^{\circ} \mathrm{F}\left(204^{\circ} \mathrm{C}\right)$, showing no evidence of pop-in.

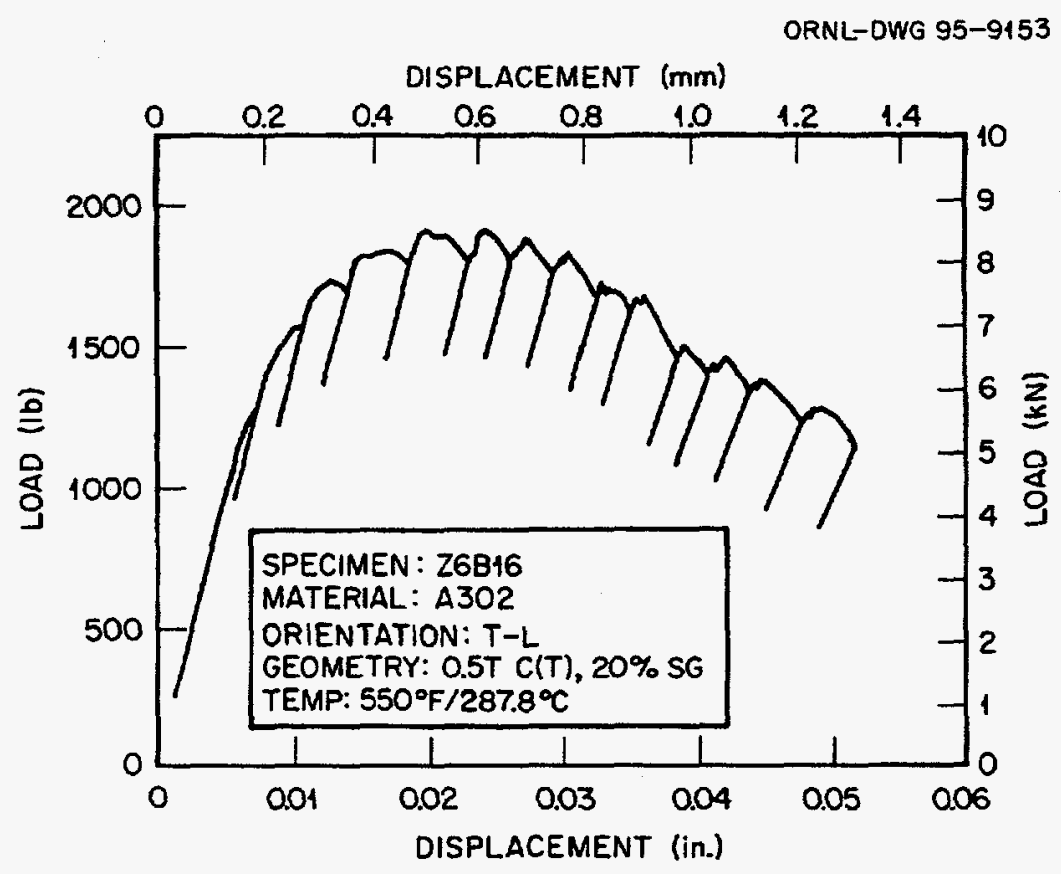

Figure 8. Load-displacement record of 1/2T compact specimen tested at $550^{\circ} \mathrm{F}\left(288^{\circ} \mathrm{C}\right)$, showing a hint of minor instability advances that can be disregarded. 


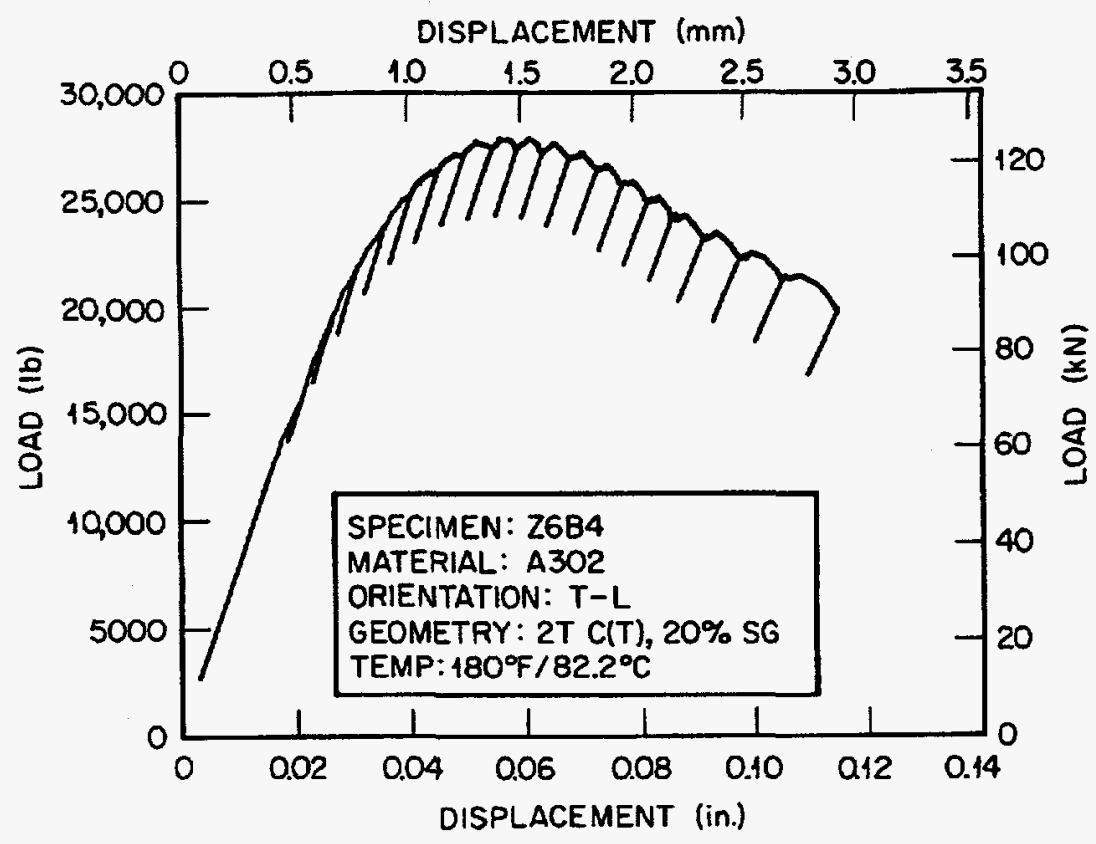

Figure 9. Load-displacement record of $2 \mathrm{~T}$ compact specimen tested at $180^{\circ} \mathrm{F}\left(82^{\circ} \mathrm{C}\right)$, an upper-shelf temperature with no evidence of pop-in.

ORNL-DWG 95-9155

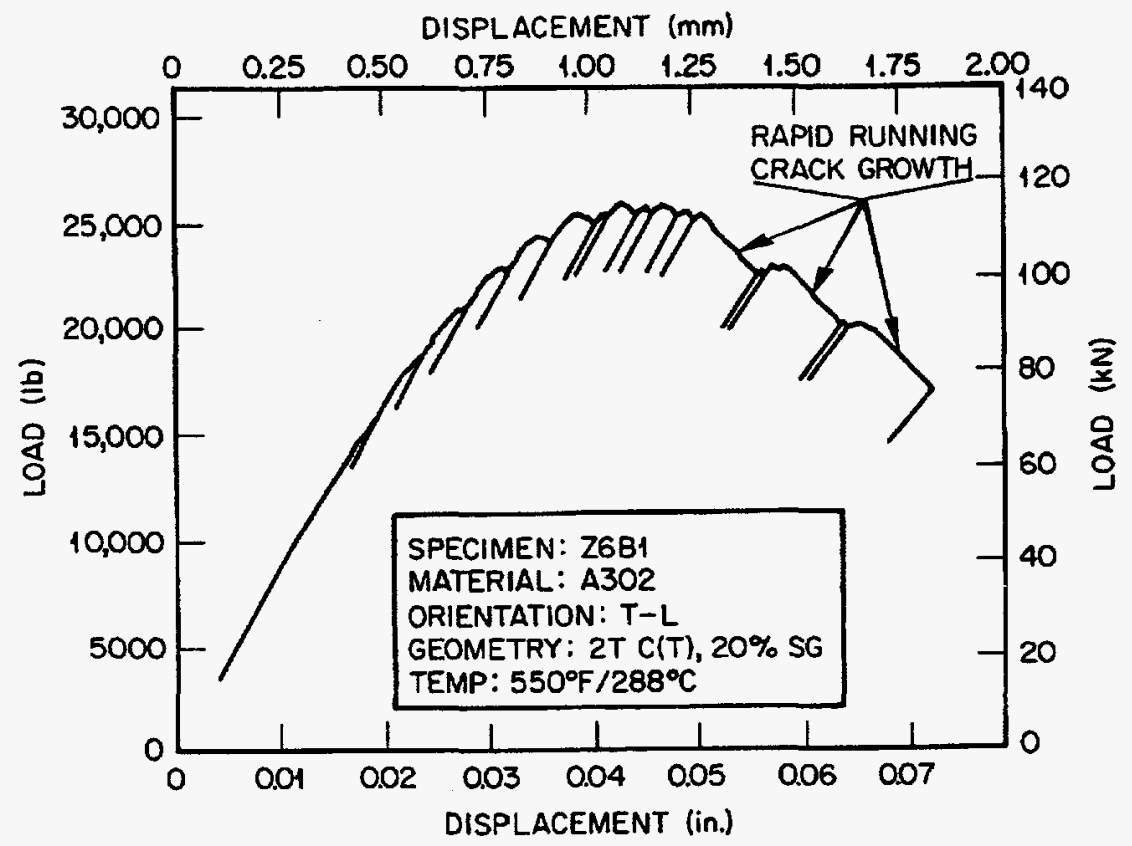

Figure 10. Load-displacement record of $2 \mathrm{~T}$ compact specimen tested at $550^{\circ} \mathrm{F}\left(288^{\circ} \mathrm{C}\right)$, showing clear evidence of pop-in behavior at upper-shelf temperature. 


\section{Comments on Available Test Standards}

The principal use for an ASTM test method or practice is to establish rules of testing such that independently operating laboratories can produce comparable data. Also, precision among replicate tests must be good enough to fairly compare one material against another. The ASTM test methods related to $J_{k}$ determination and J-R curves have been repeatedly evaluated and revised for more than a decade, and this study on modified A 302 grade B steel represents an opportunity to evaluate the progress made.

The first comparison is to evaluate the evolution of analysis procedures for J-integral at the onset of slow-stable crack growth, namely $J_{k c}$. Figure 11 compares ASTM E 813-81 to E 813-89 determinations. The 1981 version uses a straight-line fit to $\mathrm{J}-\Delta \mathrm{a}$ data exclusively within the exclusion zone indicated in Figure 4. A linear regression line fitted to these data (not shown) is projected to intersect the blunting line where $J_{k c}$ is defined. This point perhaps slightly underpredicts $J$ at the exact point of onset of ductile tearing. The 1989 version, on the other hand, identifies a point on the J-R curve at $0.008 \mathrm{in}$. $(0.2 \mathrm{~mm})$ of ductile tearing that is advanced forward from the blunting line. This revised definition had been recommended in 1983 in a users experience workshop. ${ }^{3}$ A simple power law equation is fitted to the $\mathrm{J}-\Delta \mathrm{a}$ data within the exclusion zone. Then $\mathrm{J}_{\mathrm{lc}}$ is calculated at $0.008 \mathrm{in}$. of ductile tearing on this mathematical representation of the J-R curve. Figure 11 suggests that the principal gain from this was an average increase in $\mathrm{J}_{\mathrm{lc}}$ of about $7 \%$. The precision of measurement does not seem to have been changed.

The difference between the 1989 and the 1996 versions" for $J_{k}$ determination is more subtle than the previous revision. $J_{t e}$ is still defined at 0.008 in. $(0.2 \mathrm{~mm})$ of ductile tearing beyond the blunting line. Also, both versions of the procedure use a power law curve to fit to the data in the exclusion zone and to determine $0.008 \mathrm{in}$. $(0.2 \mathrm{~mm})$ of crack growth. The principal difference is that the 1989 version had used elastic modulus adjustment in the unloading compliance equations to fit the initial part of the $\mathrm{J}-\Delta$ a test data to the calculated blunting line. Any elastic modulus that reasonably characterized the elastic properties of the test material could be used, but the investigator was enabled to apply some subjective judgment to position the data relative to the blunting line. The 1996 version for $J_{1 c}$ uses handbook elastic moduli to develop a J- $\Delta$ a data set, which is then least-squares fit with a third-order polynomial [see Figure 12(a)]. The point on the polynomial curve at which $\mathrm{J}$ is zero identifies the $\Delta \mathrm{a}=0$ point, and all $\mathrm{J}-\Delta \mathrm{a}$ data are transposed horizontally so that the polynomial fit curve and the blunting line have the same origin [see Figure 12(b)]. Elastic modulus is removed as a variable, and the 1989 subjectivity allowed for fitting to the blunting line is removed. $J_{l c}$ results from the two methods plotted against each other are presented in Figure 13. The standard deviation was determined to be about $35 \mathrm{in}$. $-\mathrm{bb} / \mathrm{in} .^{2}(6 \mathrm{kPa} \cdot \mathrm{m})$. Choosing one method (1989) and comparing variability among replicate specimens (to be covered in Section 6), the standard deviation was typically about 115 in.-lb/in. ${ }^{2}(20 \mathrm{kPa} \cdot \mathrm{m})$.

The bottom-line observation about the two revisions to the $J_{t c}$ method is that both versions contained good information about how to properly apply the compliance technique to slow-stable crack growth measurement. Unfortunately, the attention given to the precision of the analysis procedure can be overwhelmed in materials such as $A 302$ grade $B$ steel by the variability of $J_{k}$ between duplicate specimens.

"The 1996 analysis mothod to determine $\mathrm{J}_{\mathrm{k}}$ le contained in a "combined standard" that has been balloted for the 1996 Annual Book of ASTM Standards, Sect. 3, Vol. 03.01. 
ORNL-DWG 95-9155

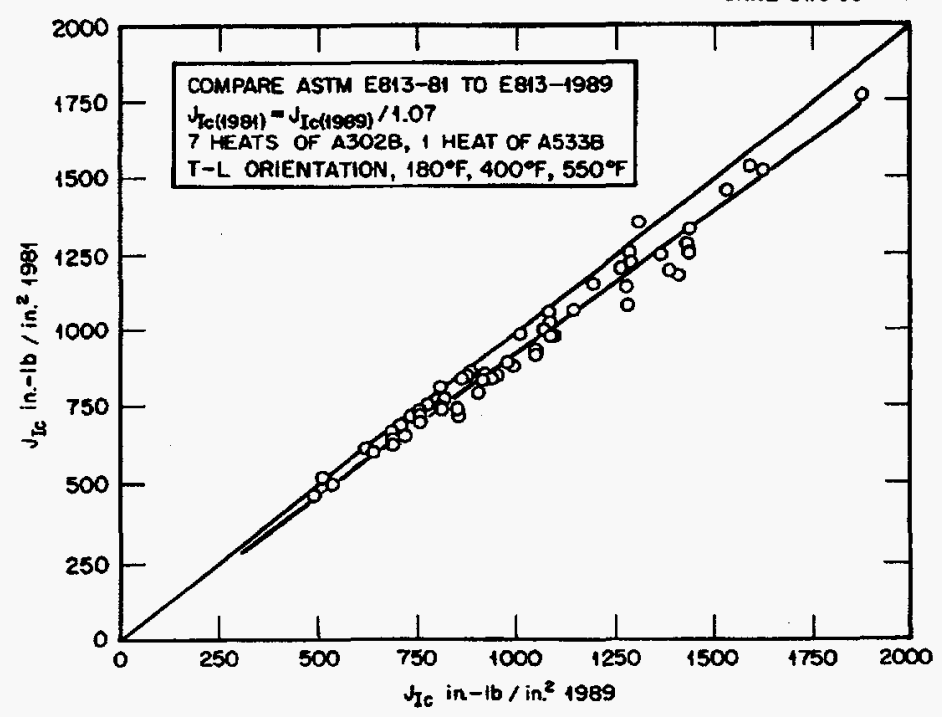

Figure 11. Comparison of the first American Society for Testing and Materials test standard on $J_{I c}$ in 1981 to the first revision made in 1989.

(a)

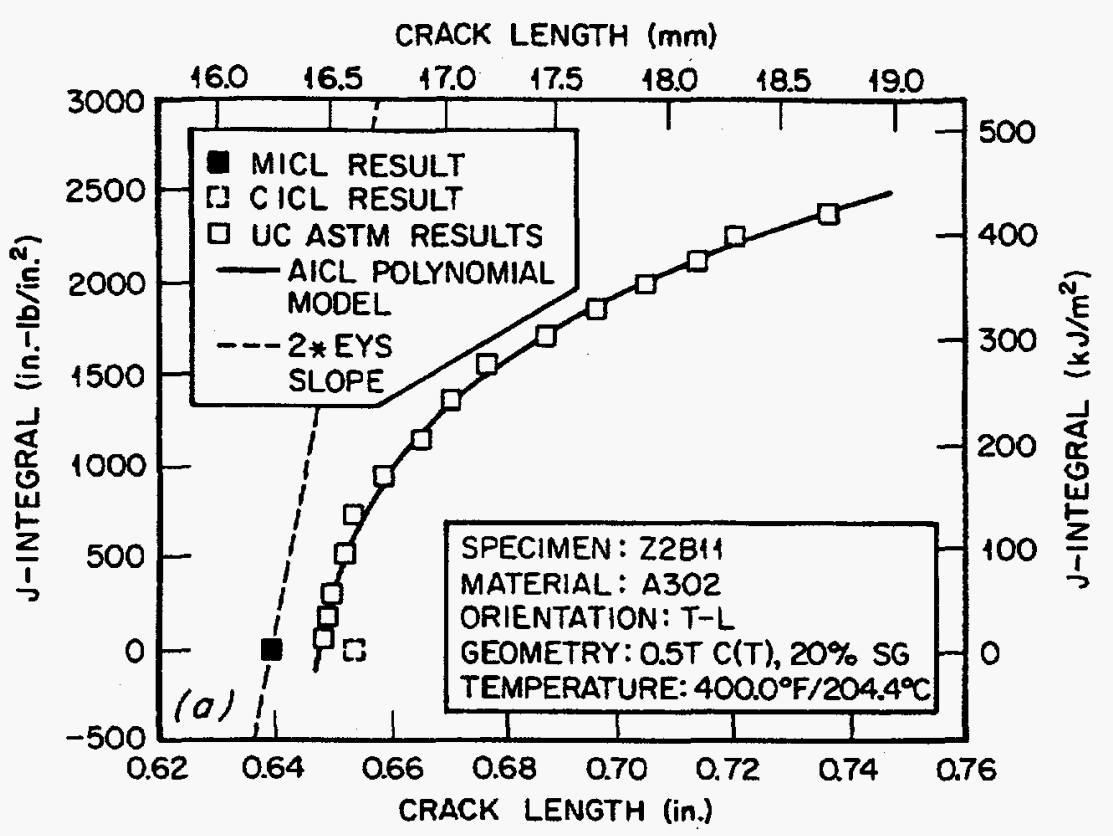

Figure 12. The 1996 American Society for Testing and Materials method (combined standard): (a) thirdorder polynomial fit and (b) adjusted $J_{R}-\Delta a$ data to the origin at the known initial physical crack size of the specimen. 
(b)

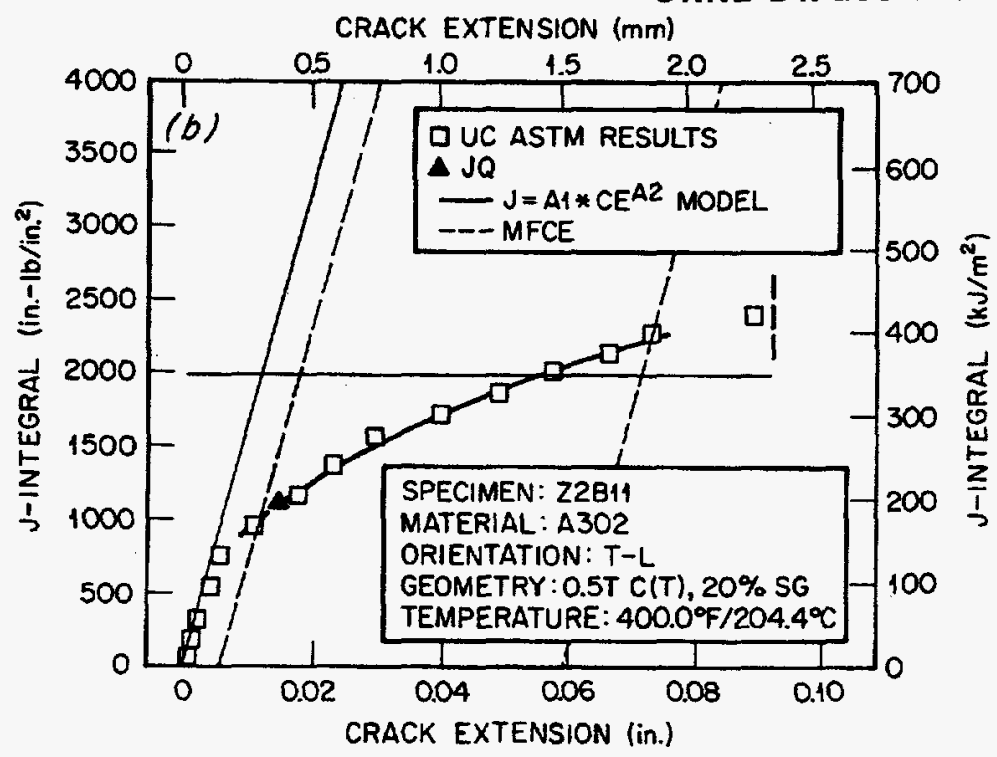

UC ASTM RESULTS-ADJUSTED DATA

$\mathrm{J}=A 1 * \mathrm{CE}^{A 2}$ - POWER LAW MODEL

MFCE-MEASURED FINAL CRACK EXTENSION.

Figure 12. (Continued).

ORNL-DWG 95-9157

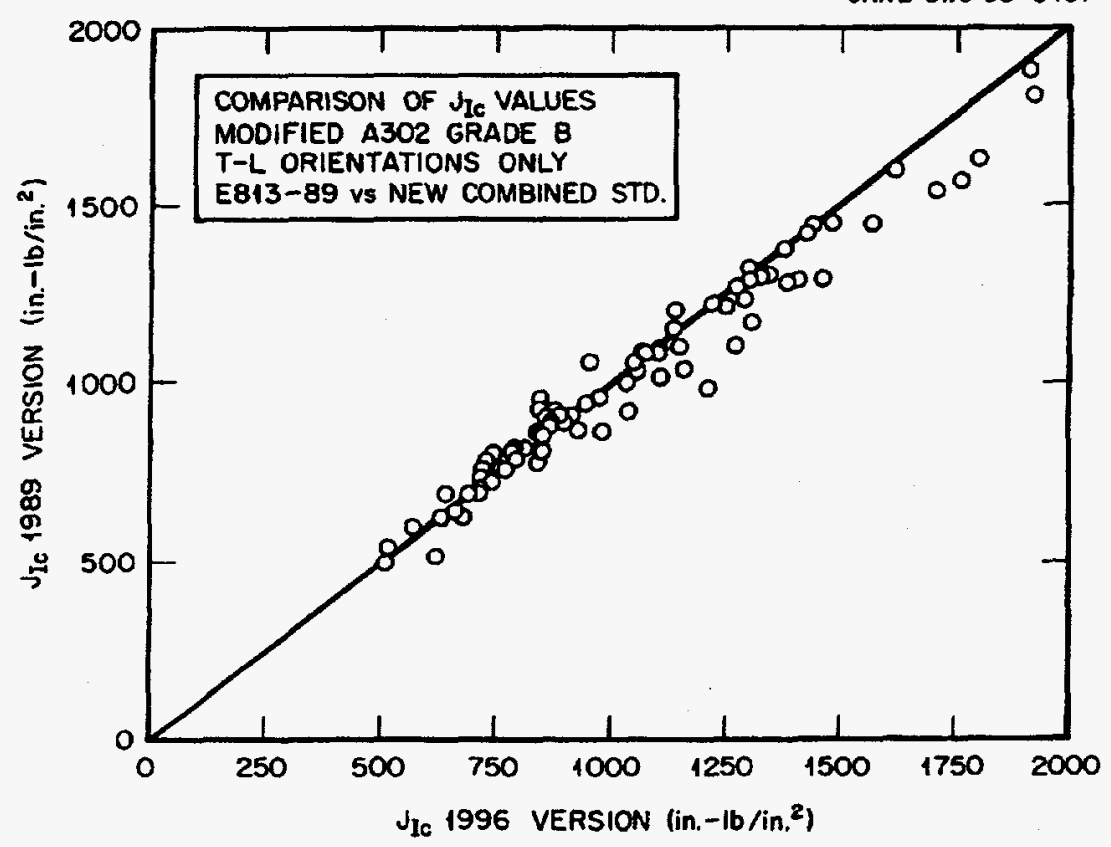

Figure 13. Comparison of the 1989 American Society for Testing and Materials $J_{\mathrm{Ic}}$ test method revision to the newly proposed 1996 version. 



\section{Material Variability}

Results from duplicate tests plotted against each other will display variability from all sources. Despite the presumed advantage of measuring $J_{k}$ from a defined point on the J-R curve, the precision of analysis seems to be unchanged since 1981. Figure 14 displays $J_{k}$ data scatter among duplicate specimens compared to $10 \%$ scatter bands parallel to the $1: 1$ correlation line. The possibility that evaluation methods alone caused the scatter can be dismissed by viewing the two J-R curves for one of the outlier points of Figure 14 (see Figures 15 and 16). Clearly, in this case, the J-R curve data are significantly different, and improvements in the precision of measurements could not remedy this lack of agreement.

The J-R curve fracture toughness parameter of $J_{0.1}$ is inherently less sensitive to the precision of crack growth measurements because this value is obtained on the part of the modified A 302 grade B J-R curves where they are tending toward an upper plateau. Hence, so long as crack growth is not overly extensive compared to the initial remaining ligament size, the $J_{0.1}$ values should provide the most discriminating comparison between duplicate specimens. This comparison is made in Figure 17. The data-pair matchups are the same as those used in Figure 14. Again, the $10 \%$ data scatter lines are applied for comparison on an equal basis. Although there are no outliers, as there were for $J_{k c}$, it appears that material variability is the dominant factor affecting reproducibility of $J_{k}$ and $J_{0.1}$ values.

The extent of J-R curve data scatter indicated in Figures 14 and 17 is relatively manageable from the standpoint of application to design and/or structural integrity analysis. Variability of another kind is demonstrated in Figure 18.

This shows the range of J-R curve toughnesses that can be obtained in modified A302 grade B plates and also lists the criteria commonly believed to be related to J-R curve. The plot includes two materials that do not belong to the above stated material identification, codes Z8 (A533 grade B class 1) and the MEA plate of code V50 (A 302 grade B). However, both have the same tensile properties as the modified A302 grade B heats. Specifically, the range of yield strengths and ultimate strengths is covered by $62 \mathrm{ksi}(427 \mathrm{MPa}) \pm 15 \%$ and $82.6 \mathrm{ksi}(569 \mathrm{MPa}) \pm 15 \%$, respectively. Total elongations are $21.7 \pm 7 \%$. One test temperature $\left(180^{\circ} \mathrm{F}\right)$ and one orientation $(T-L)$ were chosen for clarity of presentation. Clearly, tensile tests or tensile properties inferred from hardness determinations could not be used to index these J-R curves. Charpy USEs share this same deficiency to a certain extent as well. However, in the CVN case there is not a complete disconnect in relevance, but it appears that a correlation would contain extremely wide confidence bounds. Apparently, complex deformation mechanisms and responses to microstructural features interact differently between Charpy impact and fracture mechanics tests.

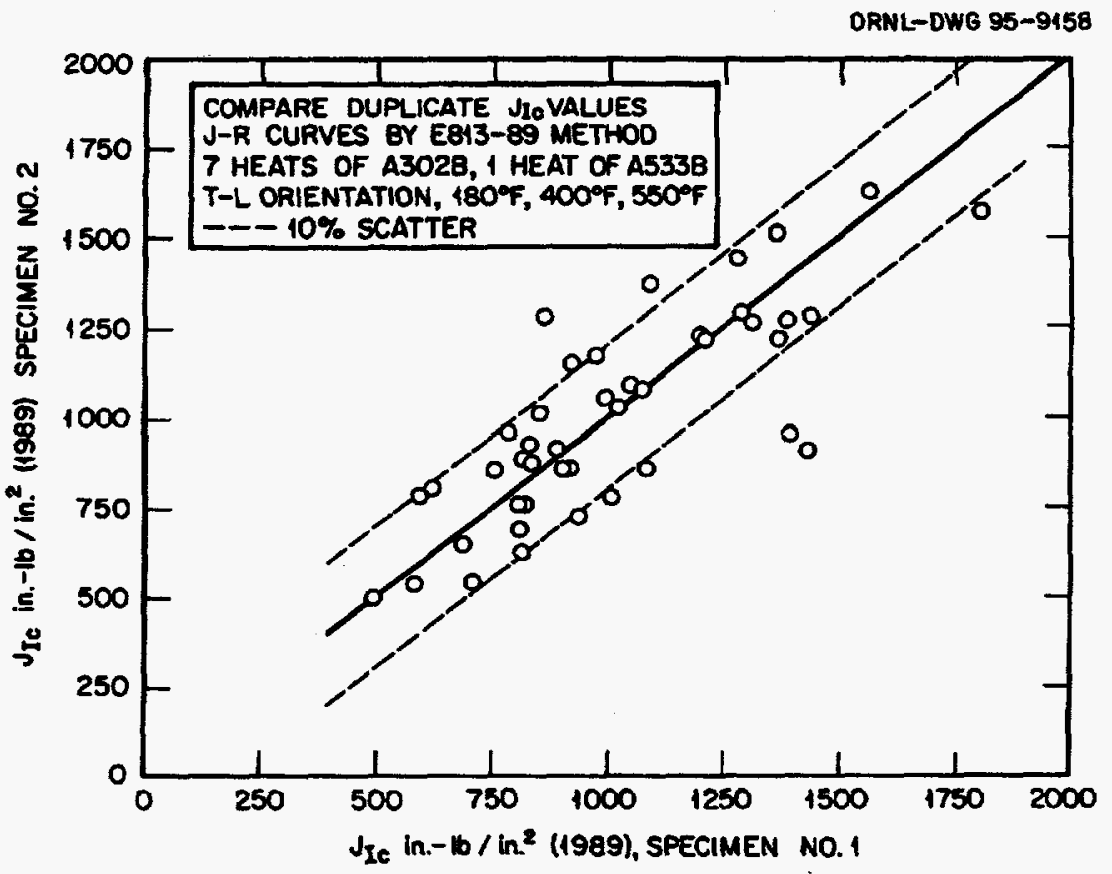

Figure 14. Data scatter of $J_{1 \varepsilon}$ due to material variability. 


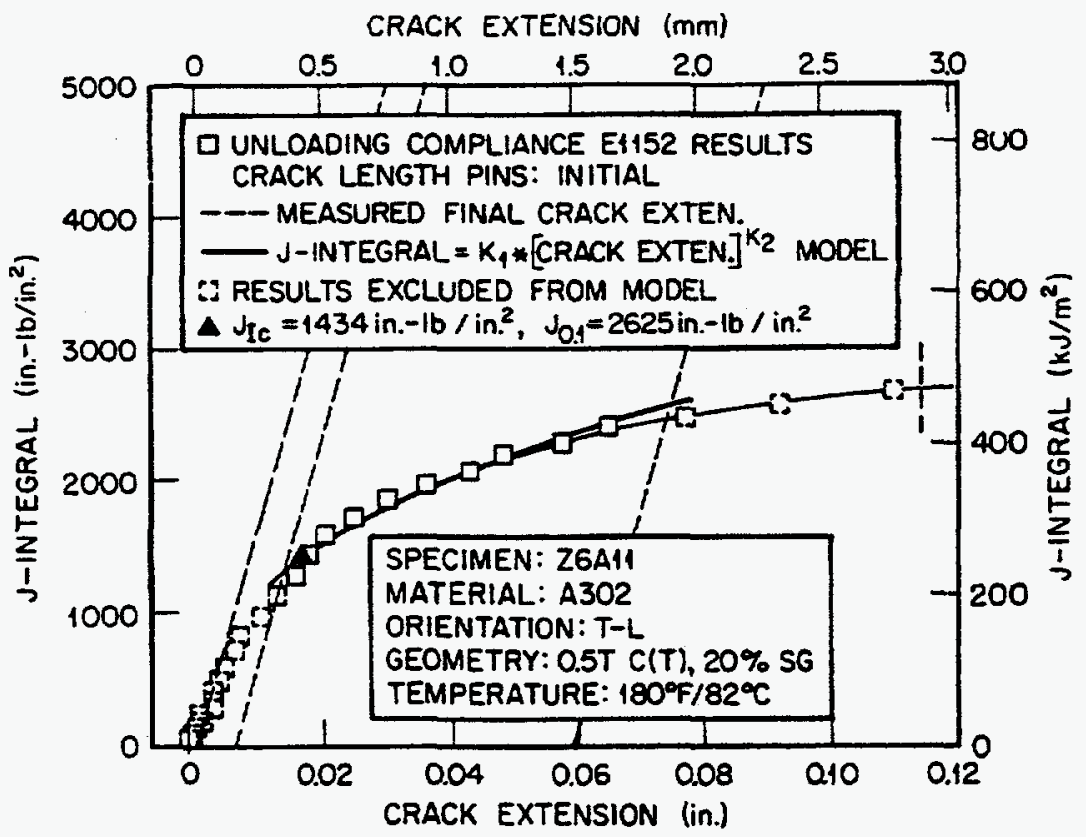

Figure 15. Typical J-R curve for comparison to the duplicate test shown in Figure 16.

ORNL-OWG 95-9161

CRACK EXTENSION (mm)

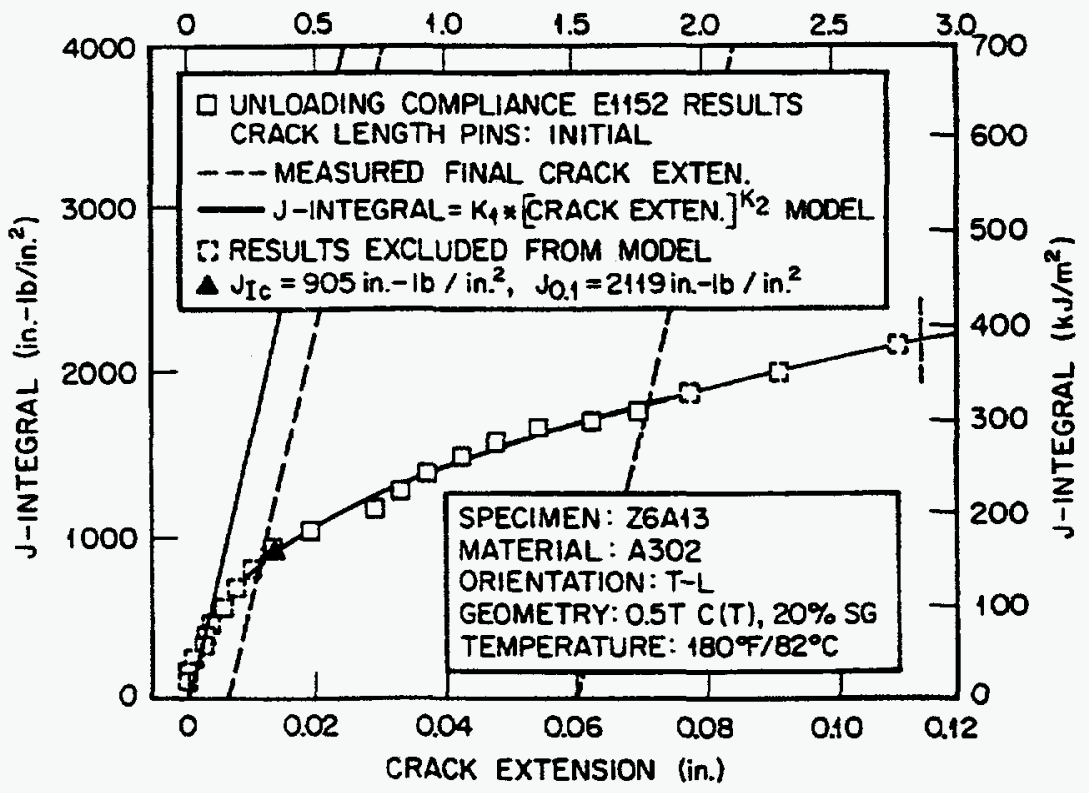

Figure 16. Typical J-R curve for comparison to the duplicate test shown in Figure 15. 


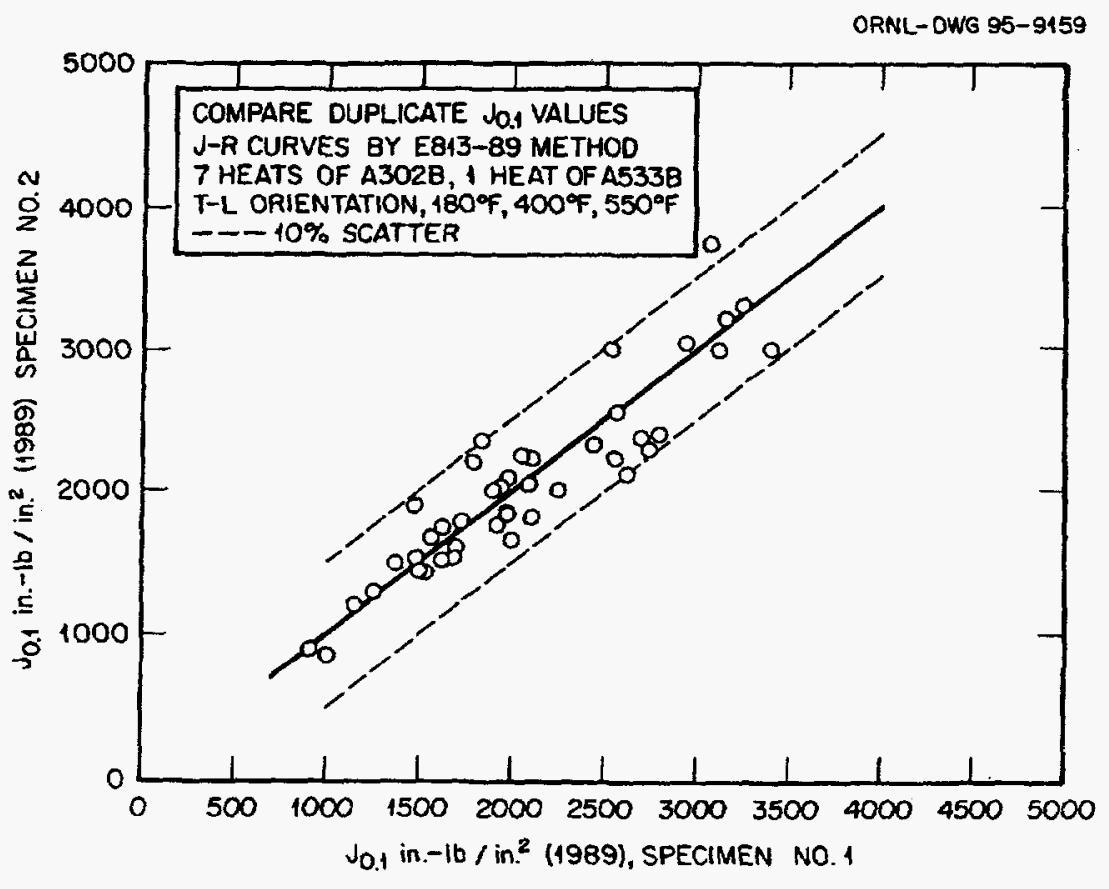

Figure 17. Data scatter of $\mathrm{J}_{0,1}$ due to material variability.

ORNL-DWG96-3456

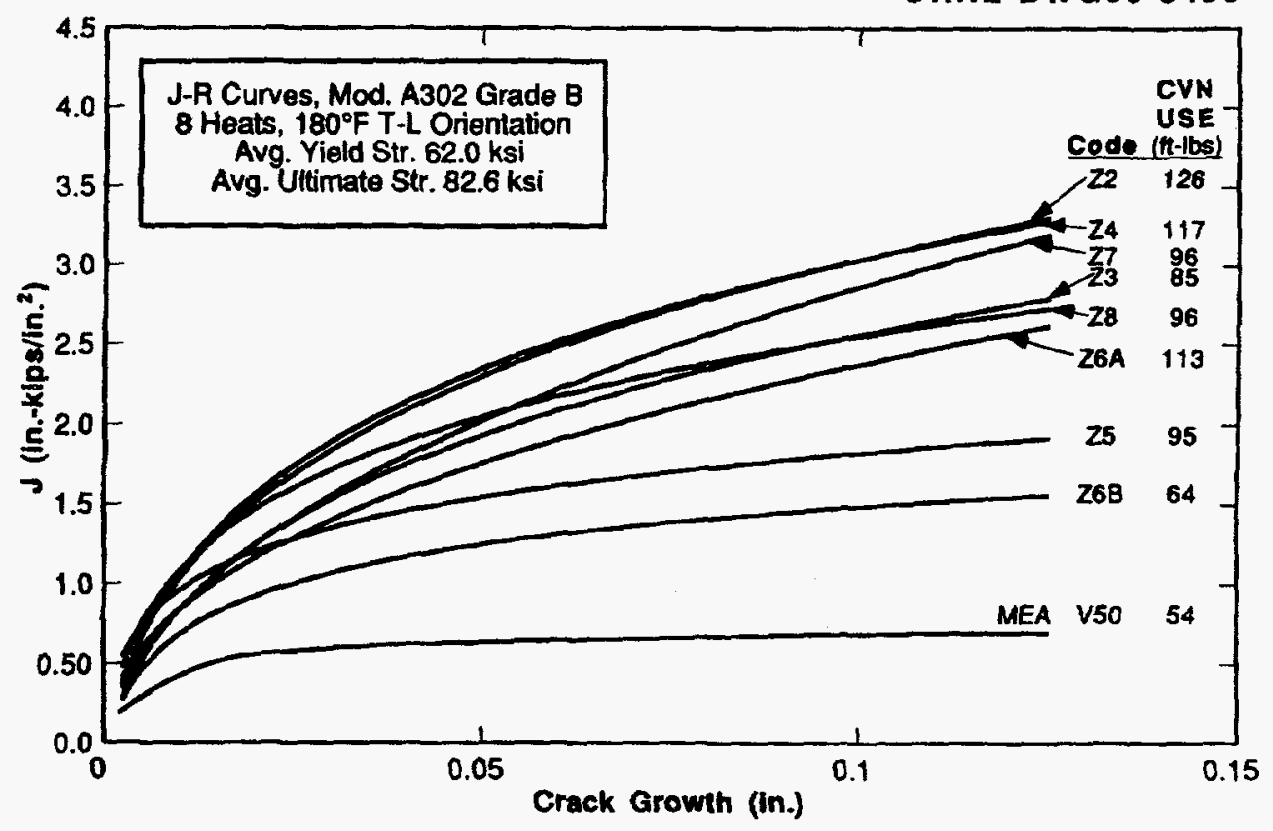

Figure 18. J-R curves for modified A302 grade B plates, one vintage A533 grade $B$ class 1 plate $(Z 8)$ and a Materials Engineering Associates plate of A302 grade B (V50). Tensile properties and corresponding Charpy V-notch impact upper-shelf energies (ft-lb) are also shown. 



\section{Conclusions}

Seven heats of modified A 302 grade $B$ plate and one vintage plate of $A 533$ grade $B$ plate steel were evaluated for upper-shelf fracture toughness in the form of J-R curves. The objectives were to (1) determine if the $J-R$ curve fracture toughness is section size-dependent, (2) develop some generic fracture toughness data on modified A 302 grade $B$ steel for potential use in analyses applications, (3) determine typical orientation effects and test temperature effects, and (4) develop generic CVN and drop-weight NDT data. Over 230 compact specimens of sizes ranging from $1 / 2 \mathrm{~T}$ to $4 \mathrm{~T}, 285 \mathrm{CVN}, 65$ tensile, and 64 drop-weight NDT specimens were tested.

It was determined that the $J-R$ curve toughness of modified A 302 grade B steel of typical production practice does not degrade with increased specimen size. The results from an earlier MEA experiment ${ }^{2}$ that showed a significant loss in J-R curve toughness with increased specimen size were not confirmed here. In fact, the fracture toughness of the MEA plate of A 302 grade B was lower than that of any of the modified A 302 grade B steel plates tested in this study, and no significant specimen size effect was observed in materials of improved toughness.

$J-R$ curve data are presented in the form of $J_{1 c}$ values, and $J$ at 0.1 in. $(2.5 \mathrm{~mm})$ of crack growth, and T-modulus. The tabulated values were averaged over all specimen sizes. Among the three orientations of transverse $(T-L)$, longitudinal $(L-T)$, and short transverse $(L-S)$, the transverse always showed the lowest toughness. The general pattern for longitudinal and short transverse directions is that both were of higher toughness than transverse, and they tended to be about equal to each other. Representative J-R curves for each orientation of each material are presented in Appendix A, and all were curve-fitted for reproduction purposes. The general pattern of test temperature effects is similarly listed from averaged data, and representative $\mathrm{J}$-R curves were fitted for presentation in Appendix B. The fact that all heats showed J-R curve toughness loss with test temperature increase to $550^{\circ} \mathrm{F}$ $\left(288^{\circ} \mathrm{C}\right)$ is typical.

Drop-weight NDT tests were fairly consistent among heats, ranging from -30 to $-5^{\circ} \mathrm{F}\left(-34\right.$ to $\left.-20^{\circ} \mathrm{C}\right)$. Evaluations for $R T_{N D T}$ showed that two heats, one modified $A 302$ grade $B$ and the vintage $A 533$ grade $B$, had insufficient CVN toughness at NDT $+60^{\circ} \mathrm{F}\left(33^{\circ} \mathrm{C}\right)$ for $R T_{N D T}$ to equal the NDT temperature. The penalty in $R T_{\text {NDT }}$ was 43 and $44^{\circ} \mathrm{F}$ $\left(24^{\circ} \mathrm{C}\right)$.

The multiple number of tests on one grade of steel provided an opportunity to evaluate $J-R$ curve and $J_{\mid c}$ test methods that have been developed and modified within ASTM. These methods have never been subjected to such a wide range of material characteristics and test conditions. The bottom-line finding from this experiment on the $J_{i c}$ method revisions made over a decade of development has the following two aspects: The establishment of test practice requirements to obtain crack growth by compliance was useful. On the other hand, the revisions to test record analysis subsequent to the 1981 version of $E \mathbf{8 1 3}$ have made the determination of $J_{\mathrm{lc}}$ unnecessarily complex and hence less useful. The revisions showed no beneficial impact on the precision of $\mathrm{J}_{\mathrm{lc}}$ determination for modified A 302 grade B steels.

Two parameters of material crack growth resistance measurement that were useful for comparisons were $T$-modulus and $J$ at 0.1 in. $(2.5 \mathrm{~mm})$ of crack growth. Each required a definition specific to this report, since neither parameter is covered by an ASTM standard analysis procedure. 



\section{References}

1. ASME Section XI Task Group on Reactor Vessel Integrity Requirements, White Paper on Reactor Vessel Integrity Requirements for Level A and B Conditions, EPRI TR-100251, Final Report, Electric Power Research Institute, Palo Alto, Calif., January 1993.

2. A. L. Hiser and J. B. Terrell, Materials Engineering Associates, Inc., Lanham, Md., Size Effects on J-R Curves for A302-B Plate, USNRC Report NUREG/CR-6265 (MEA-2320), January 1989. ${ }^{\dagger}$

3. "Standard Terminology Relating to Fracture Testing," E 616-89, pp. 647-57 in Annual Book of ASTM Standards, Vol. 03.01, American Society for Testing and Materials, Philadelphia, 1993."

4. "Fracture Toughness Requirements for Material," Sect. III, Div. 1, NB-2300, ASME Boiler and Pressure Vessel Code. An American National Standard, American Society of Mechanical Engineers, New York, 1994."

5. "Standard Test Method for $\mathrm{J}_{\mathrm{lc}}$, a Measure of Fracture Toughness," $\mathrm{E}$ 813-89, pp. 738-52 in Annual Book of ASTM Standards, Vol. 03.01, American Society for Testing and Materials, Philadelphia, 1993.

6. "Evaluation Procedures for Levels A and B Service Loading," Sect. XI, Article K-4000, ASME Boiler and Pressure Vessel Code. An American National Standard, American Society of Mechanical Engineers, New York, 1994."

7. E. D. Eason, J. E. Wright, and E. E. Nelson, Modeling and Computing Services, Boulder, Colo., Multivariable Modeling of Pressure Vessel and Piping J-R Data, USNRC Report NUREG/CR-5729 (MCS-910401), May 1991. ${ }^{\dagger}$

8. G. P. Gibson and S. G. Druce, "Some Observations on J-R Curves," pp. 166-82 in Elastic-Plastic Fracture Test Methods (The User's Experience), ASTM STP 856, American Society for Testing and Materials, Philadelphia, 1985."

Available in public technical libraries.

${ }^{\dagger}$ Available for purchase from National Technical Information Service, Springfield, VA 22161. 
Appendix A

Selected J-R Curves Representing Typical Values of T Slope, $J_{0.1}$, and $J_{1 c}$ from Table 7 



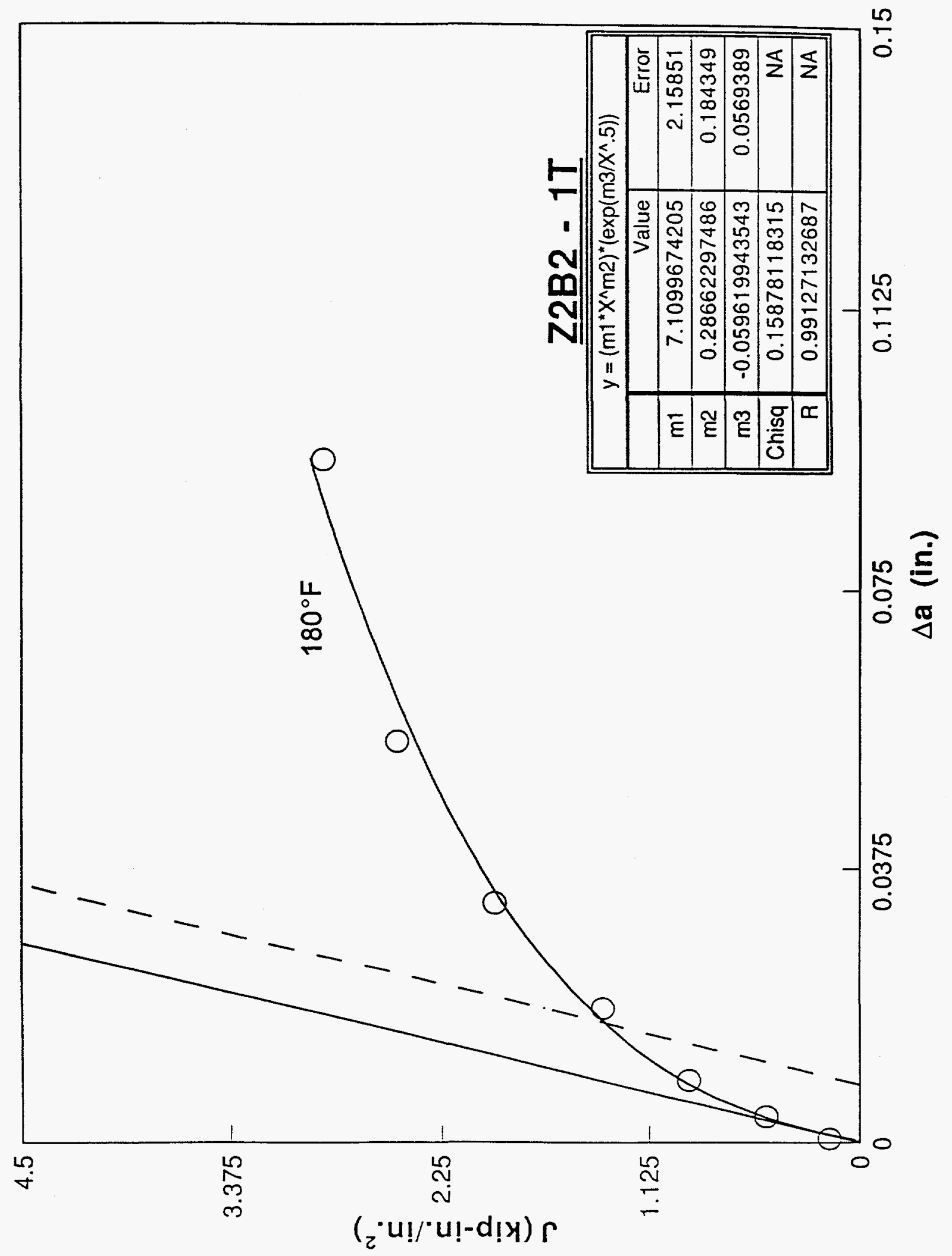




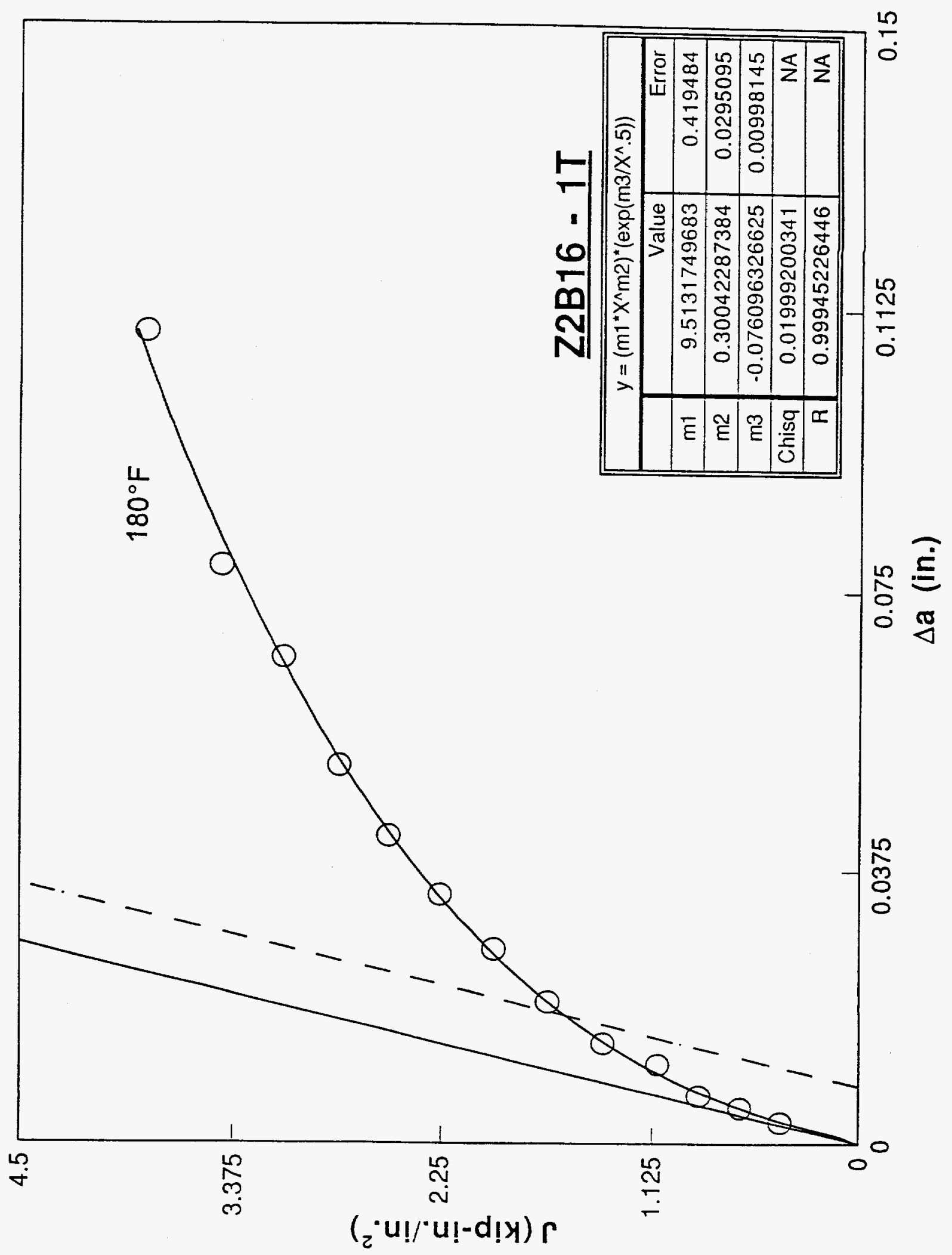




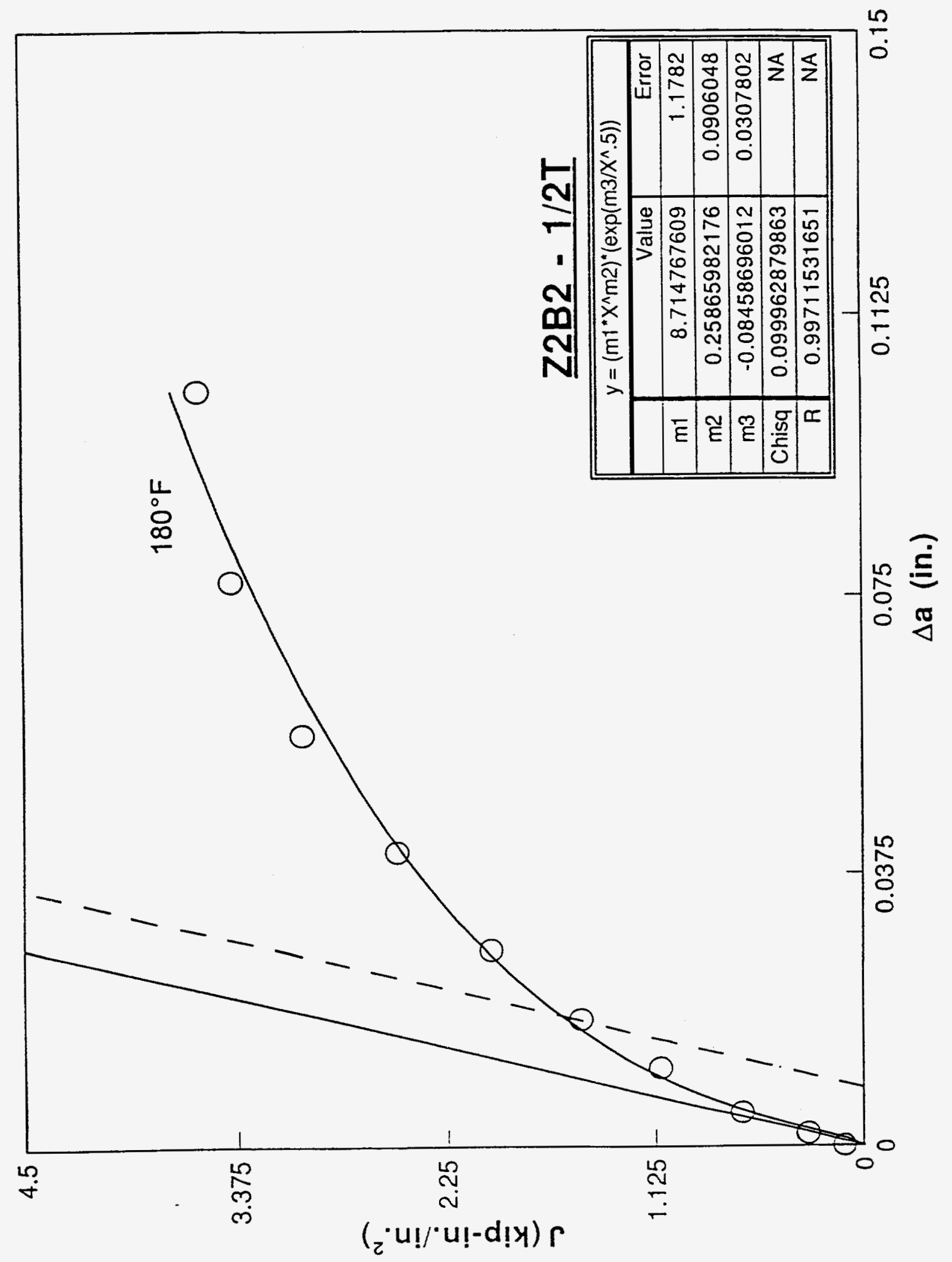




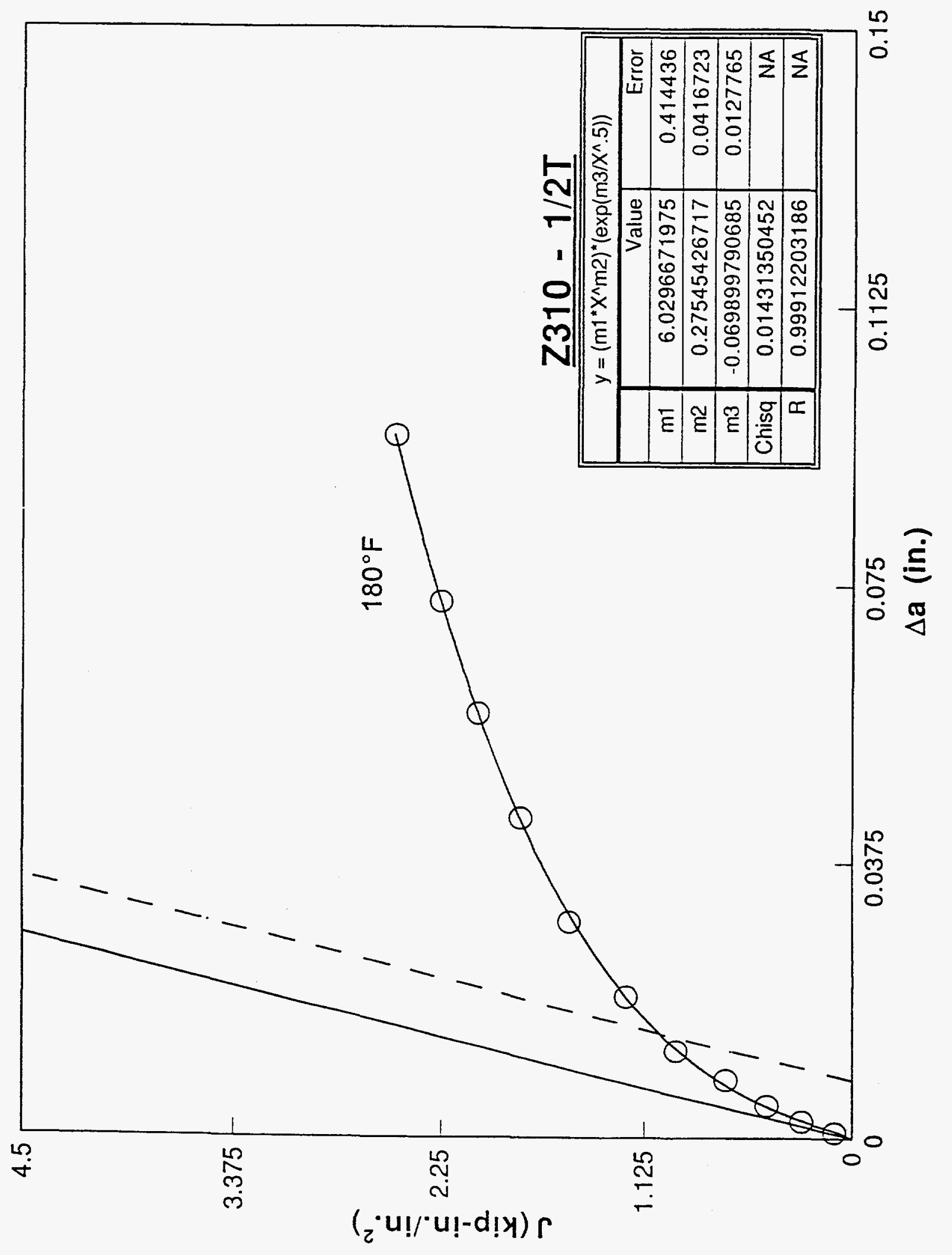




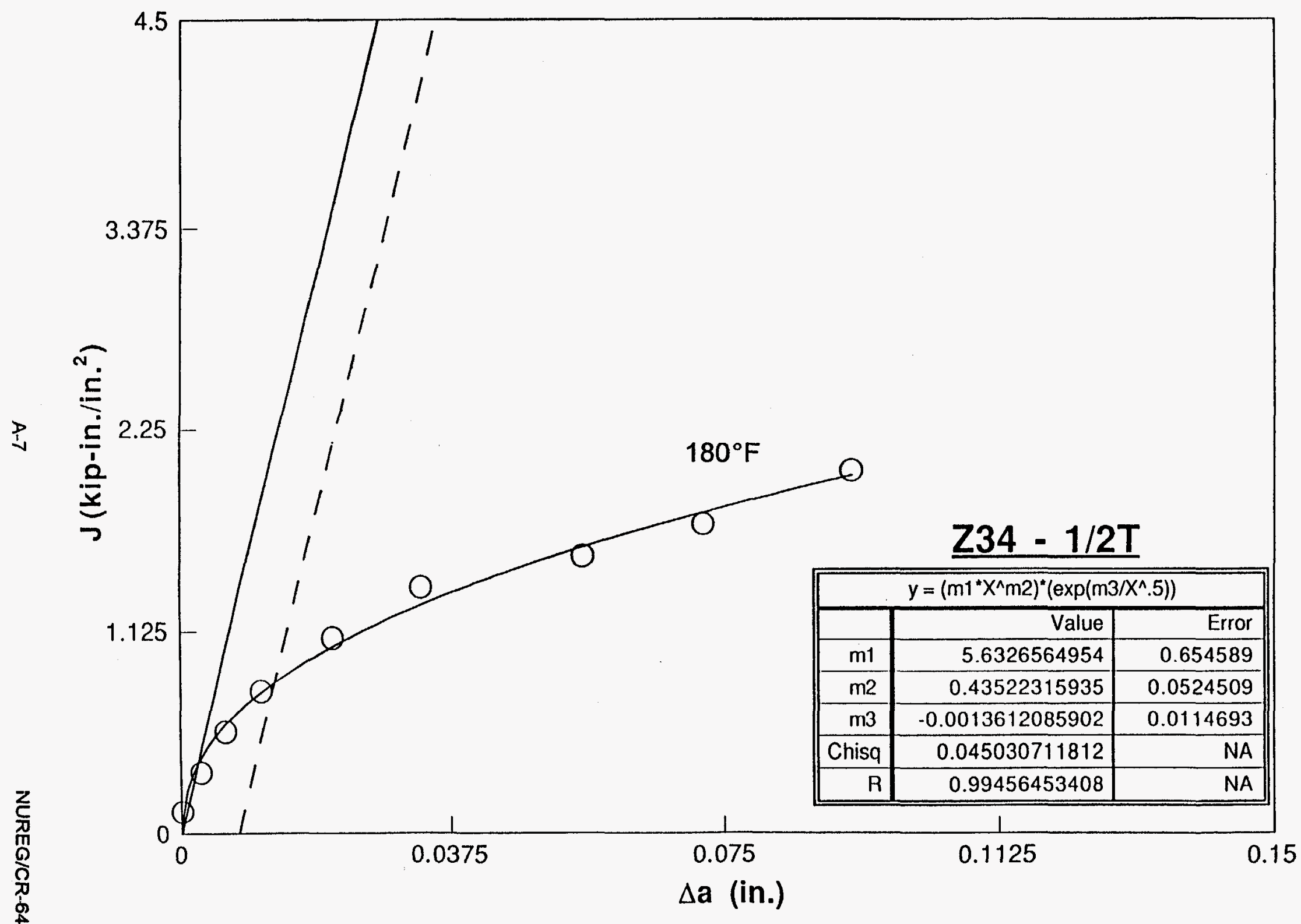




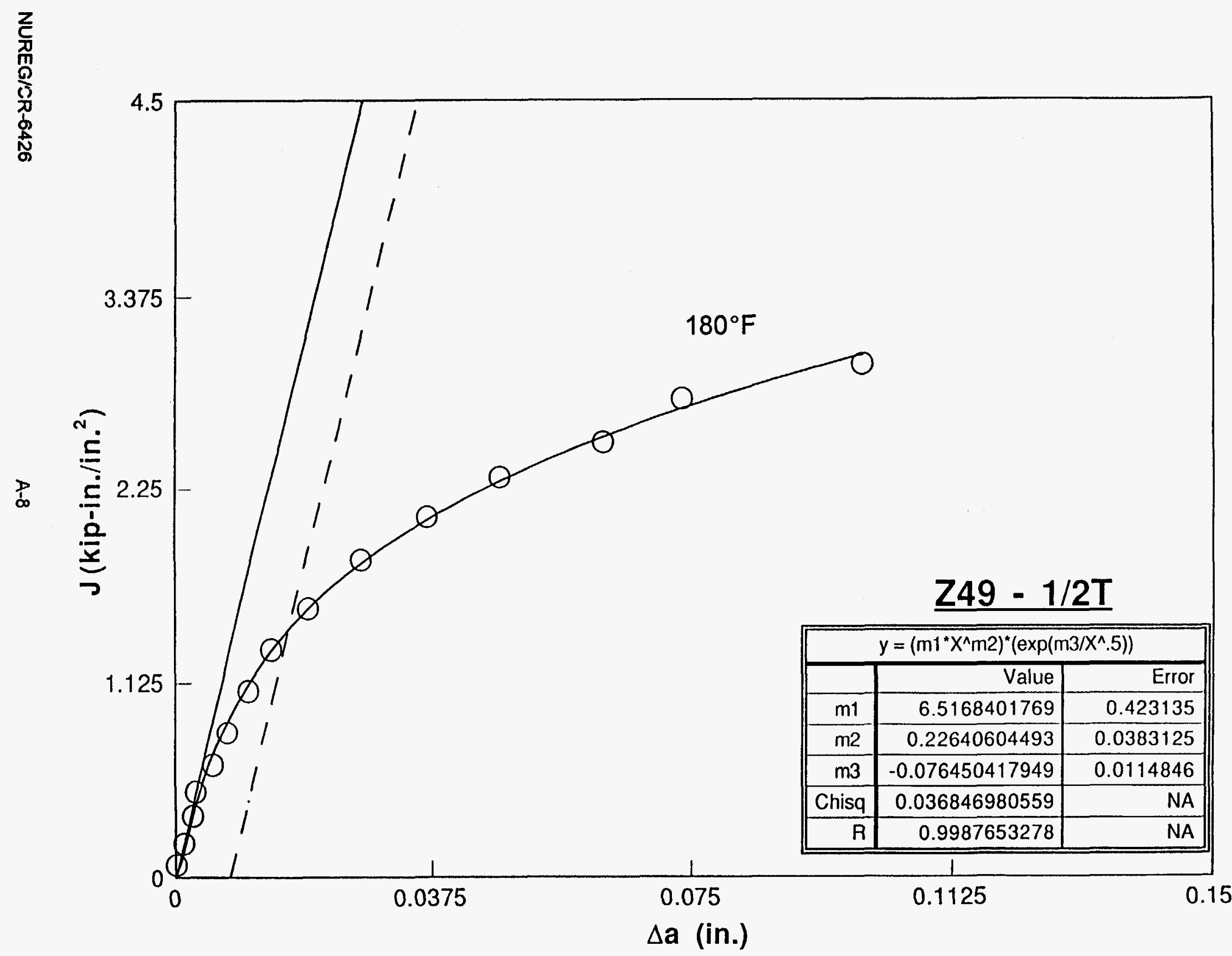




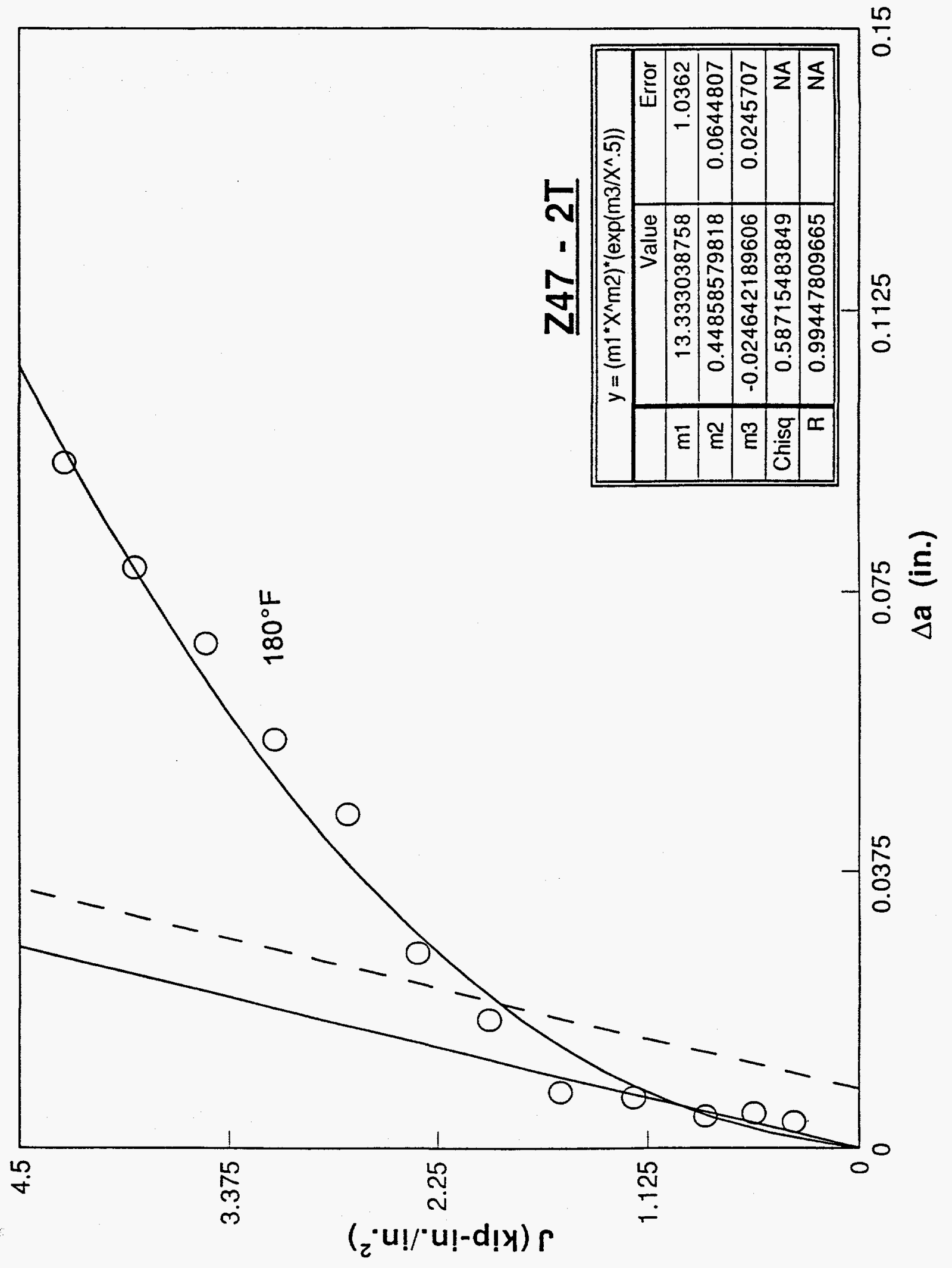




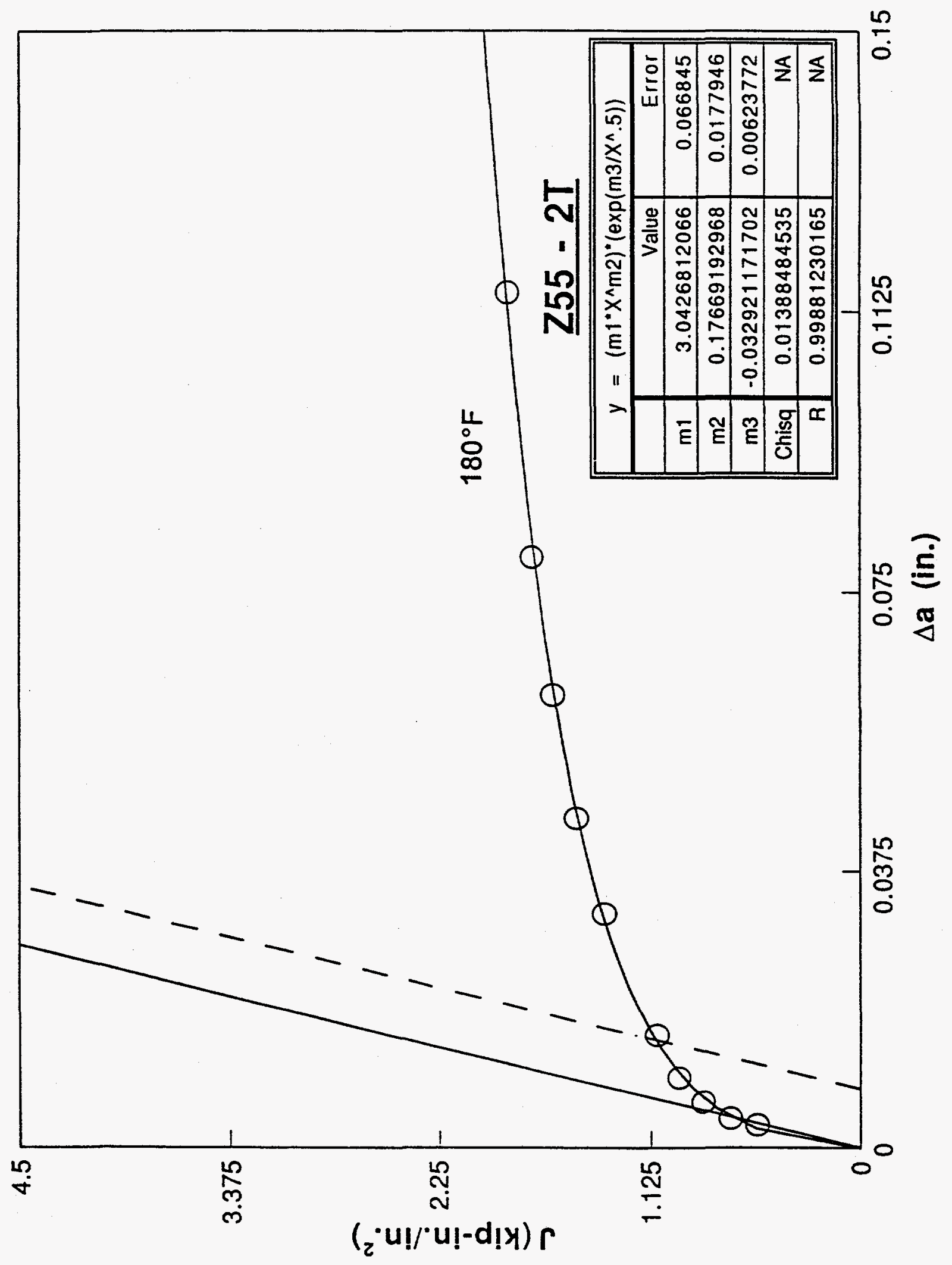




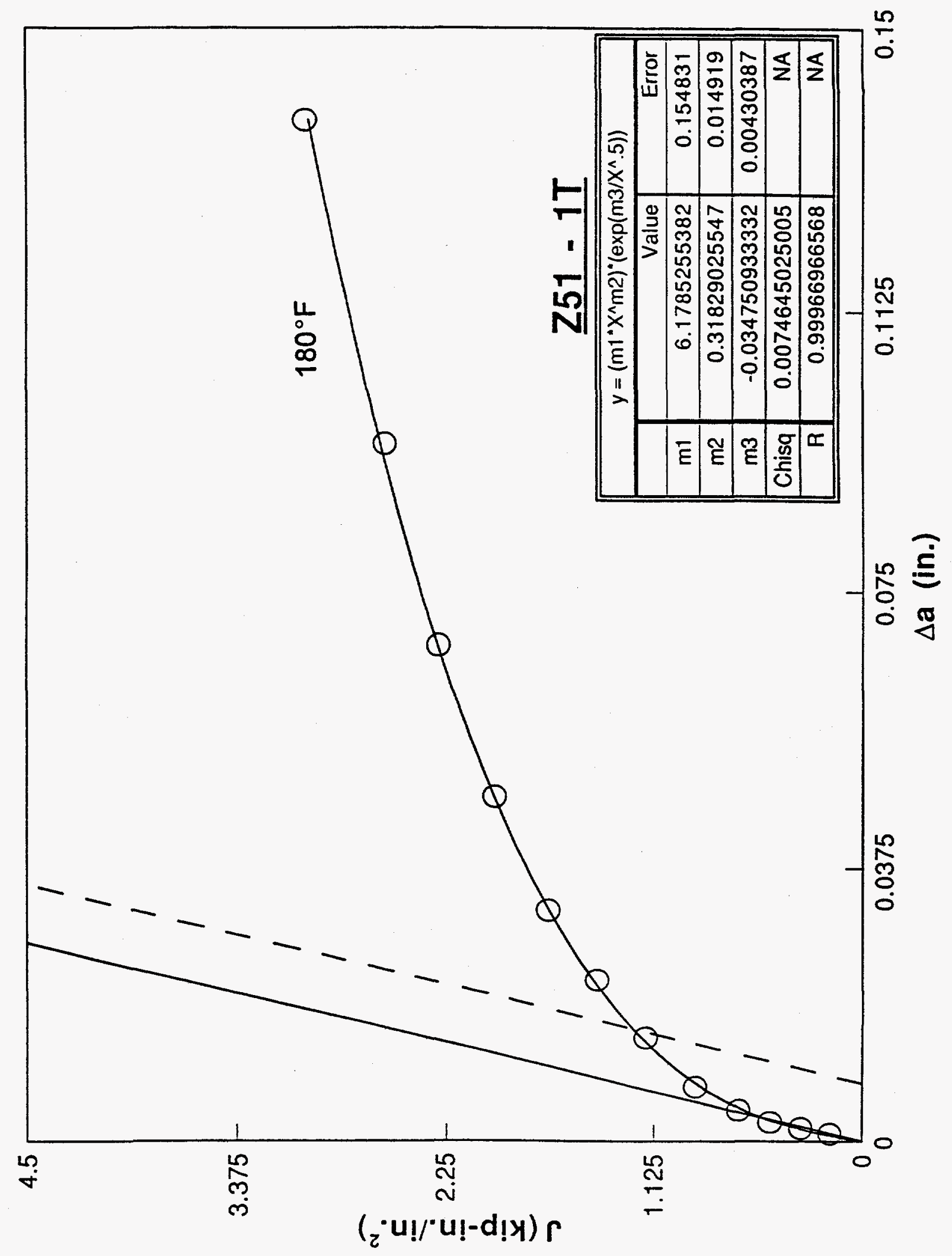




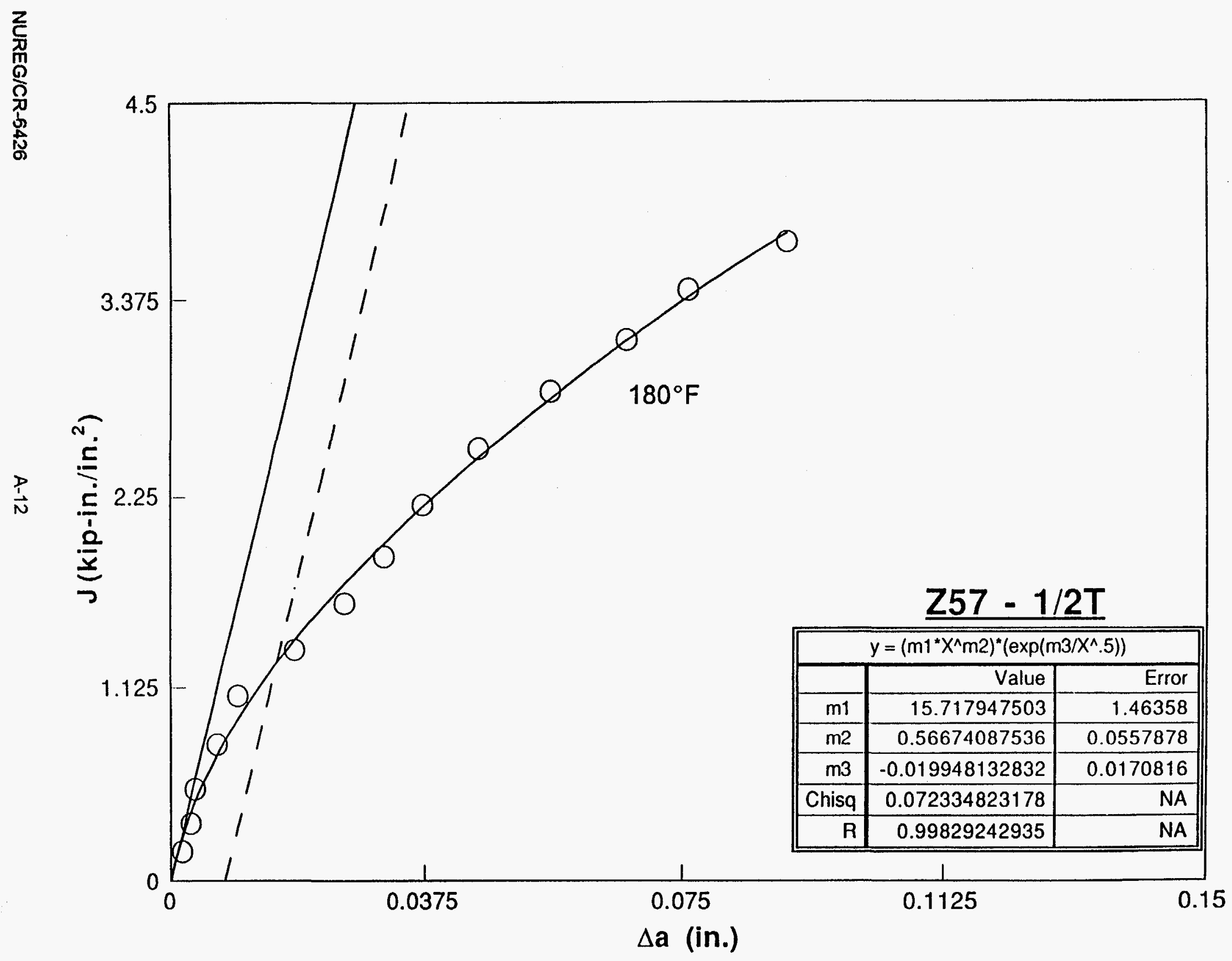




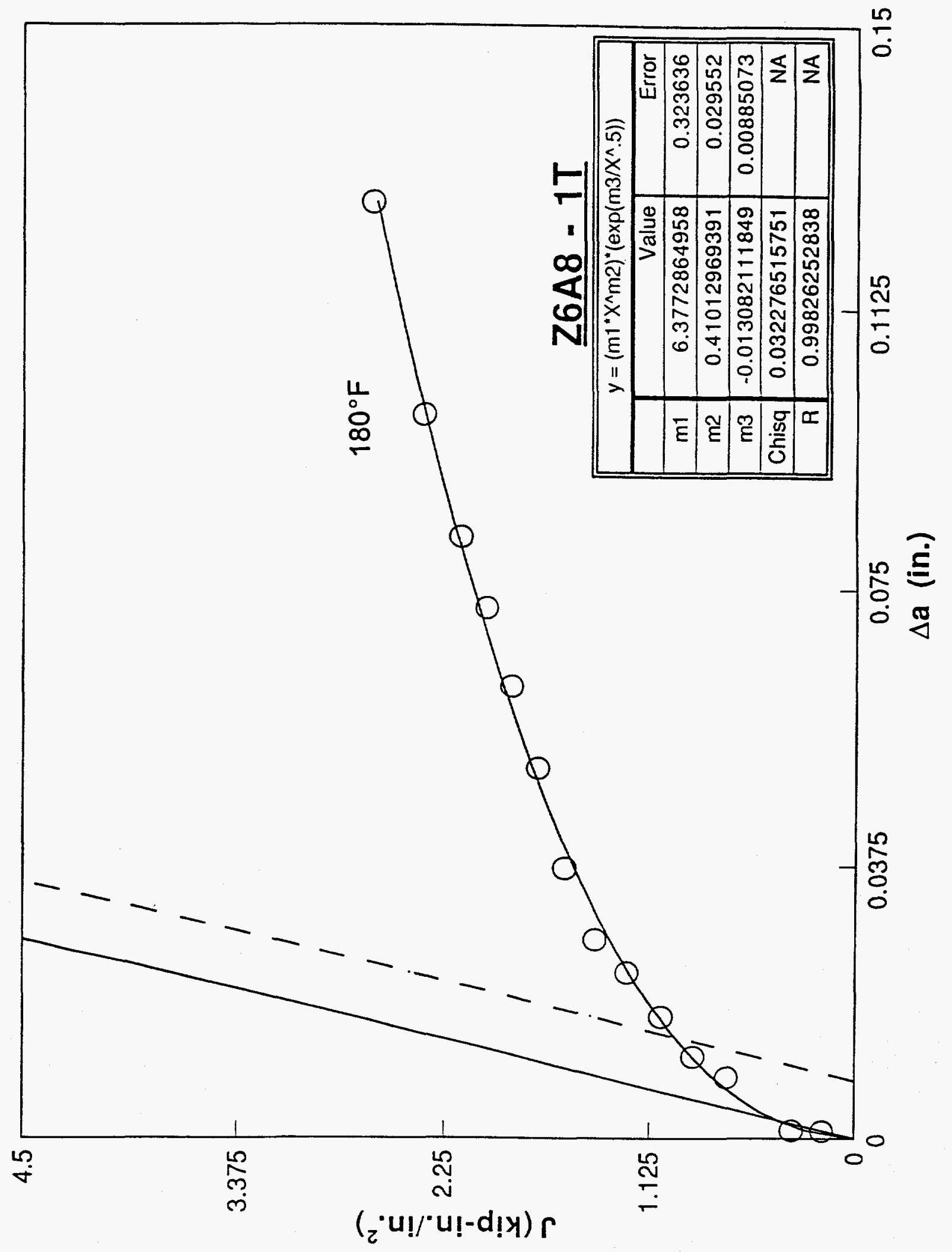




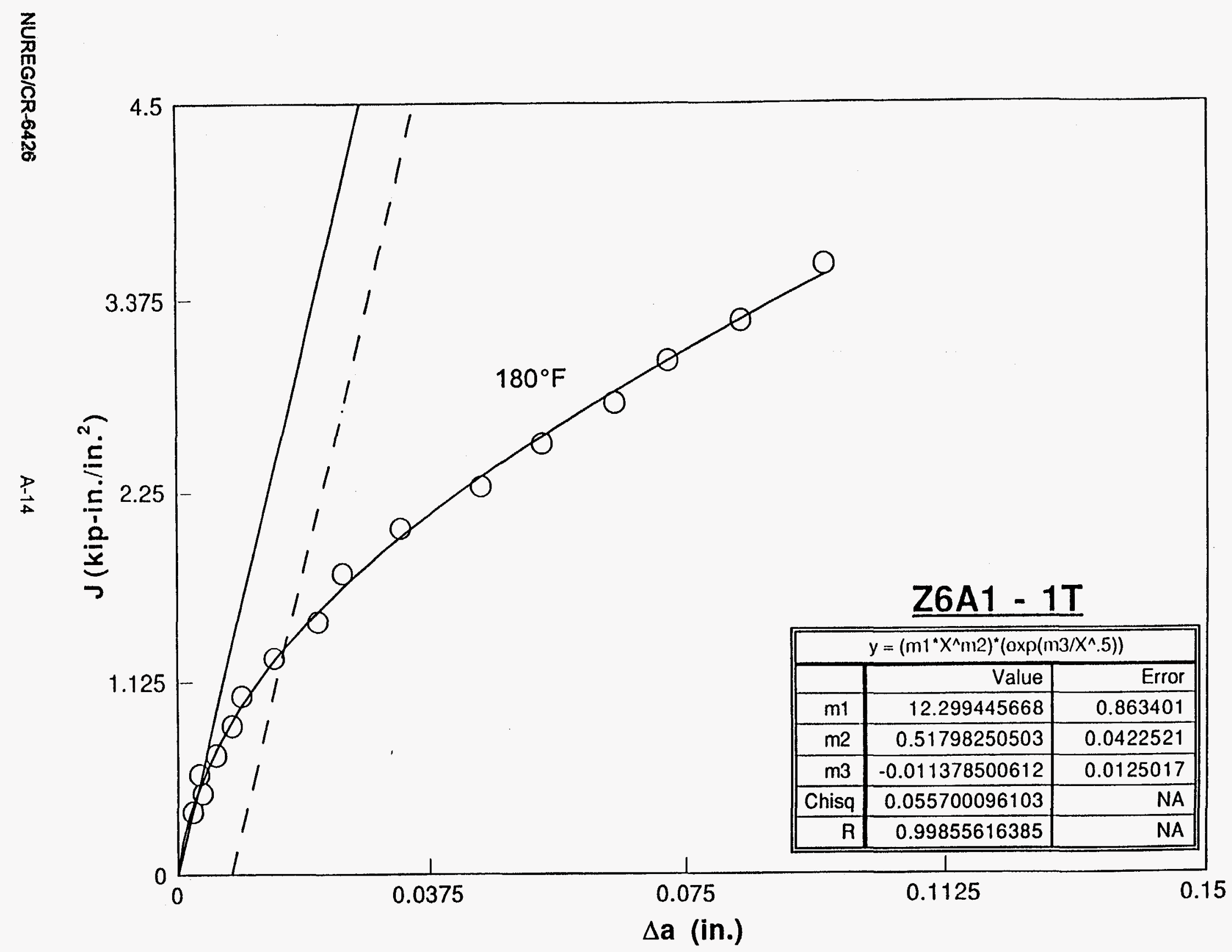




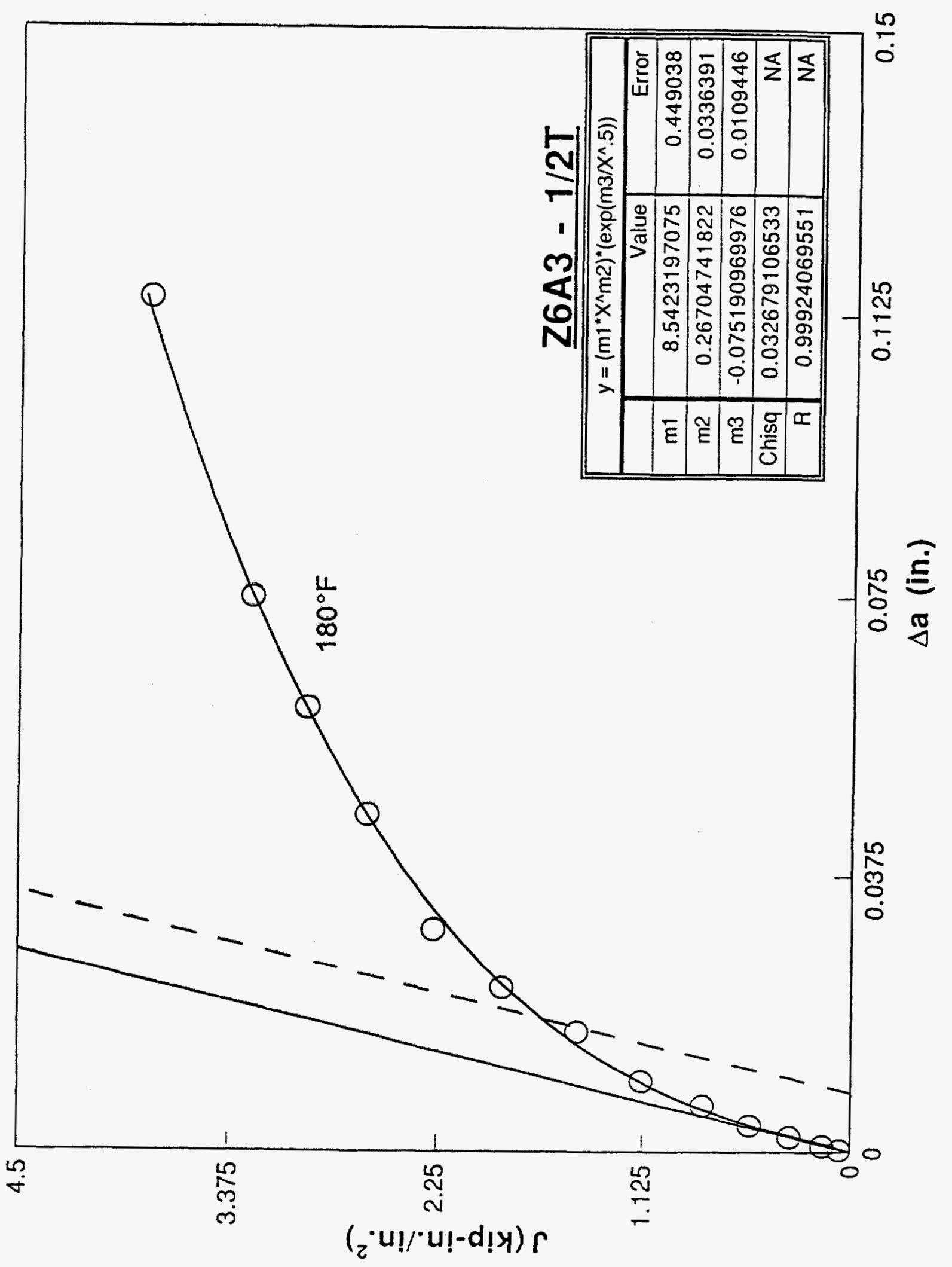




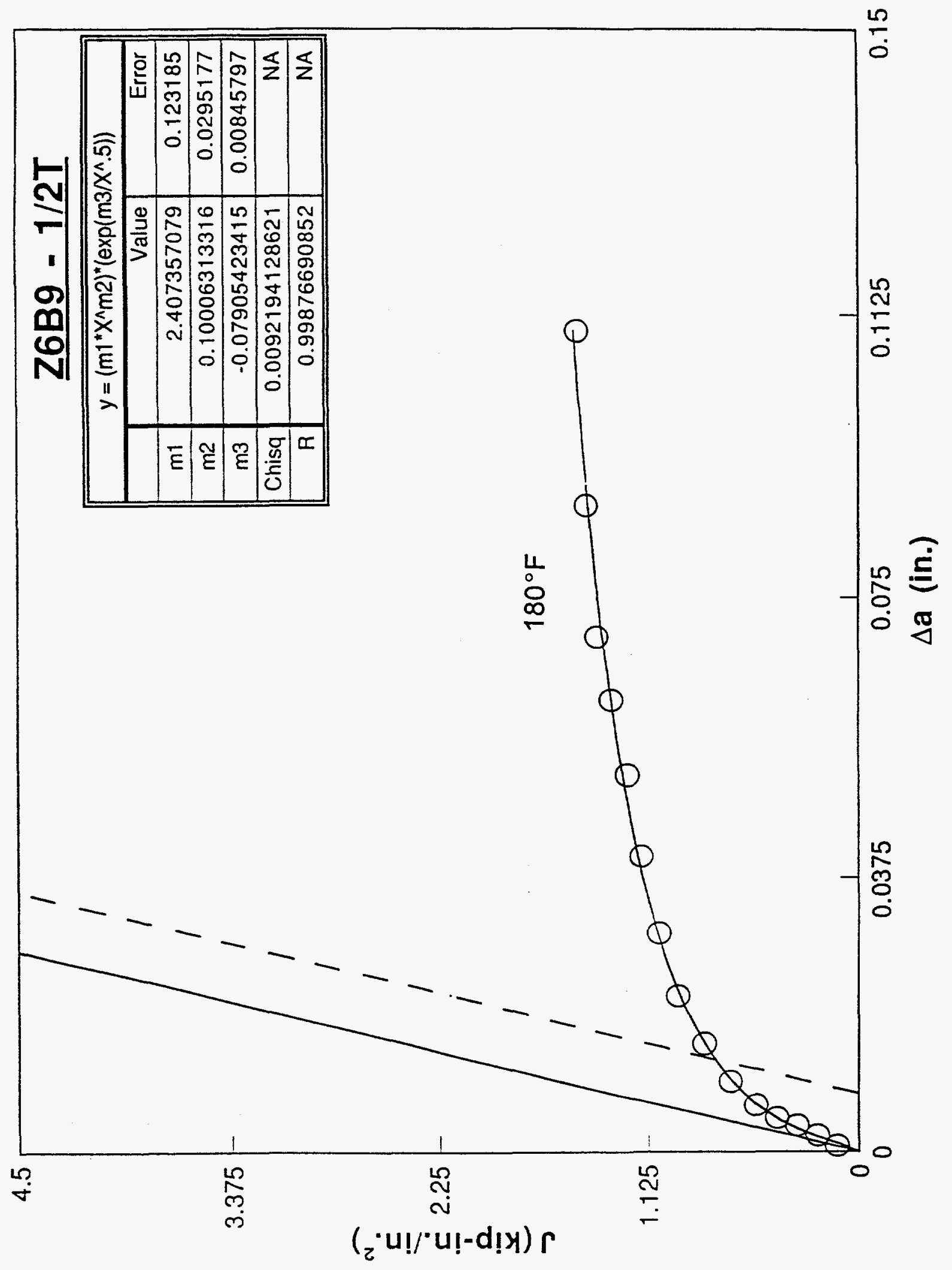




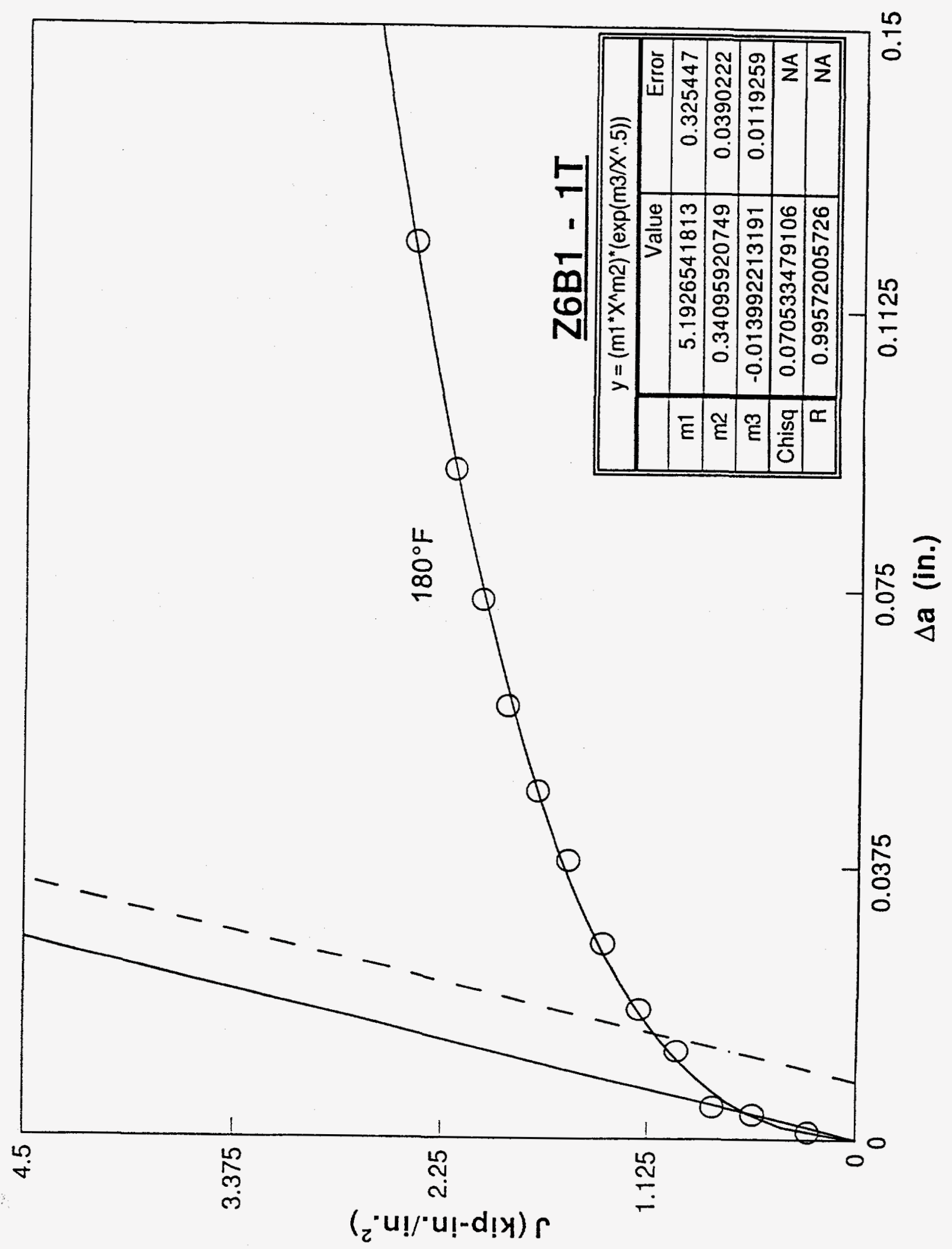




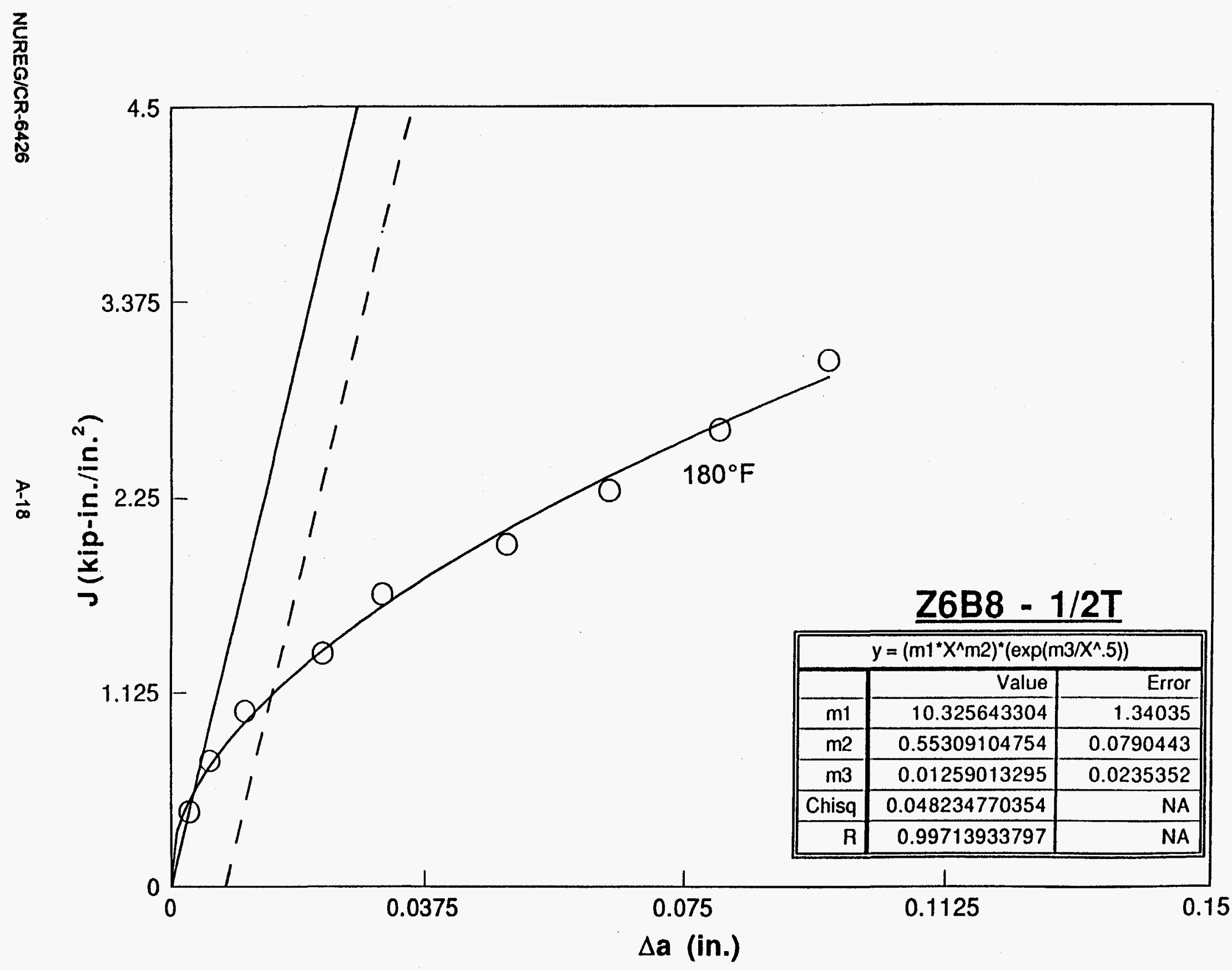




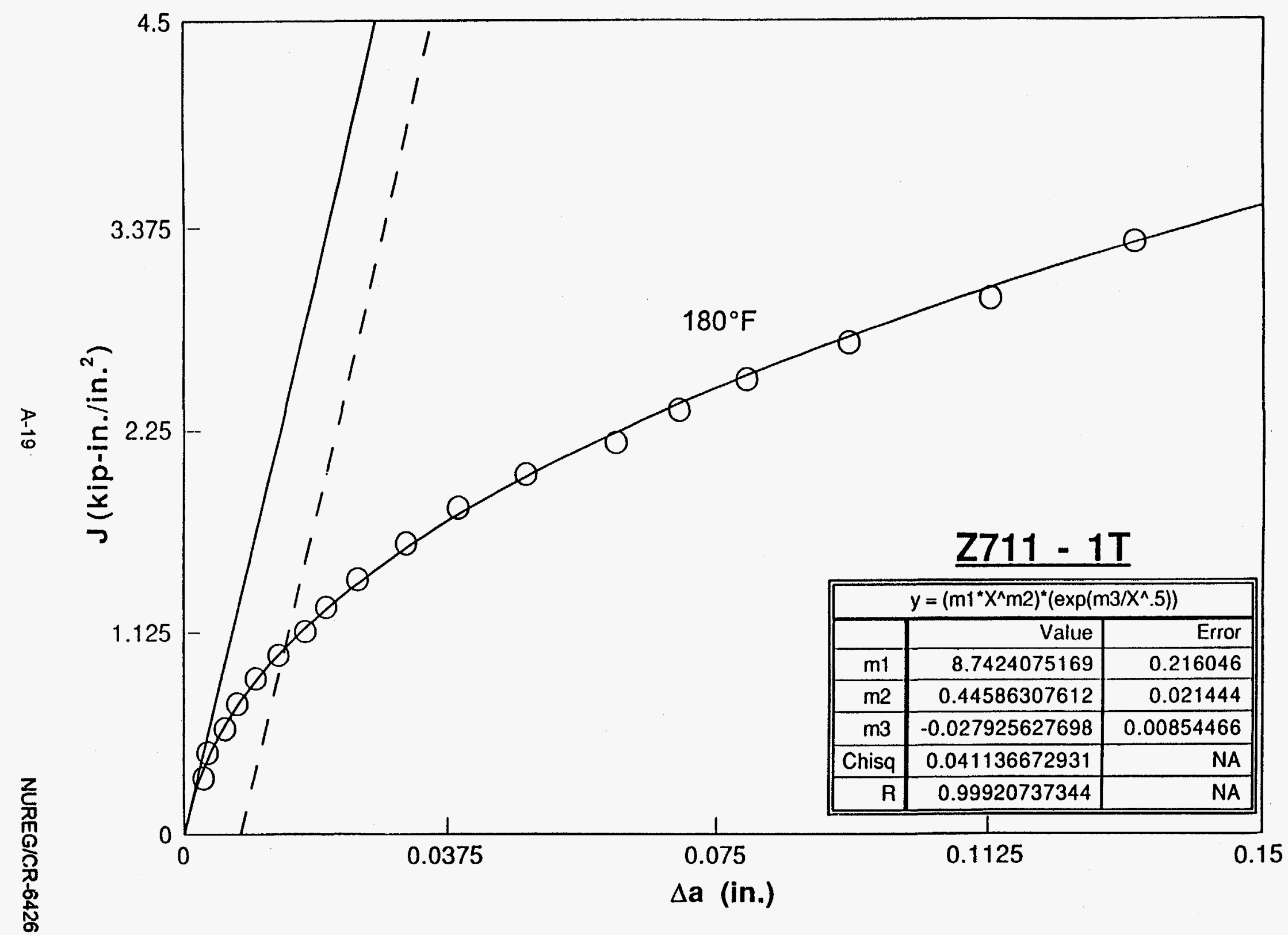




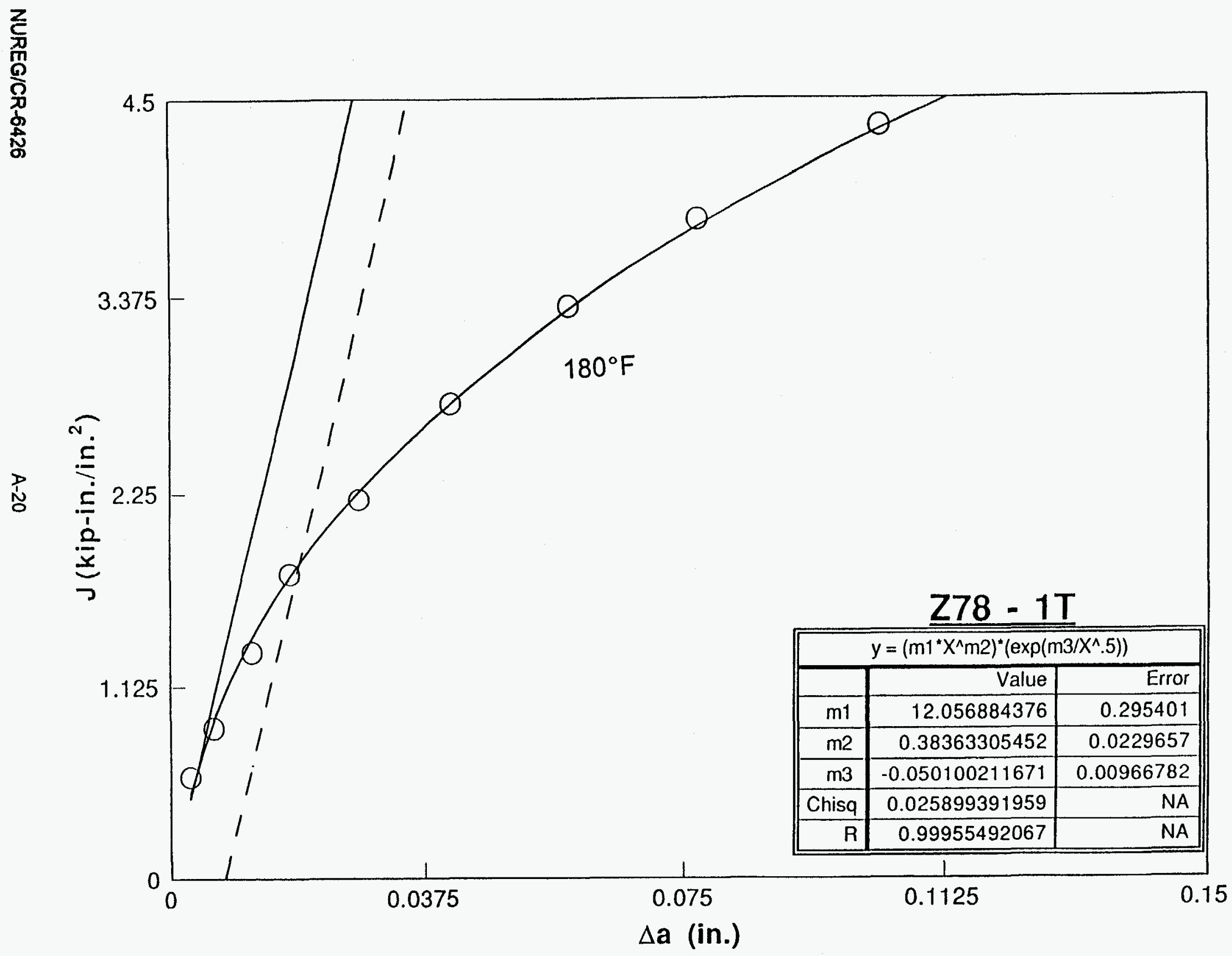




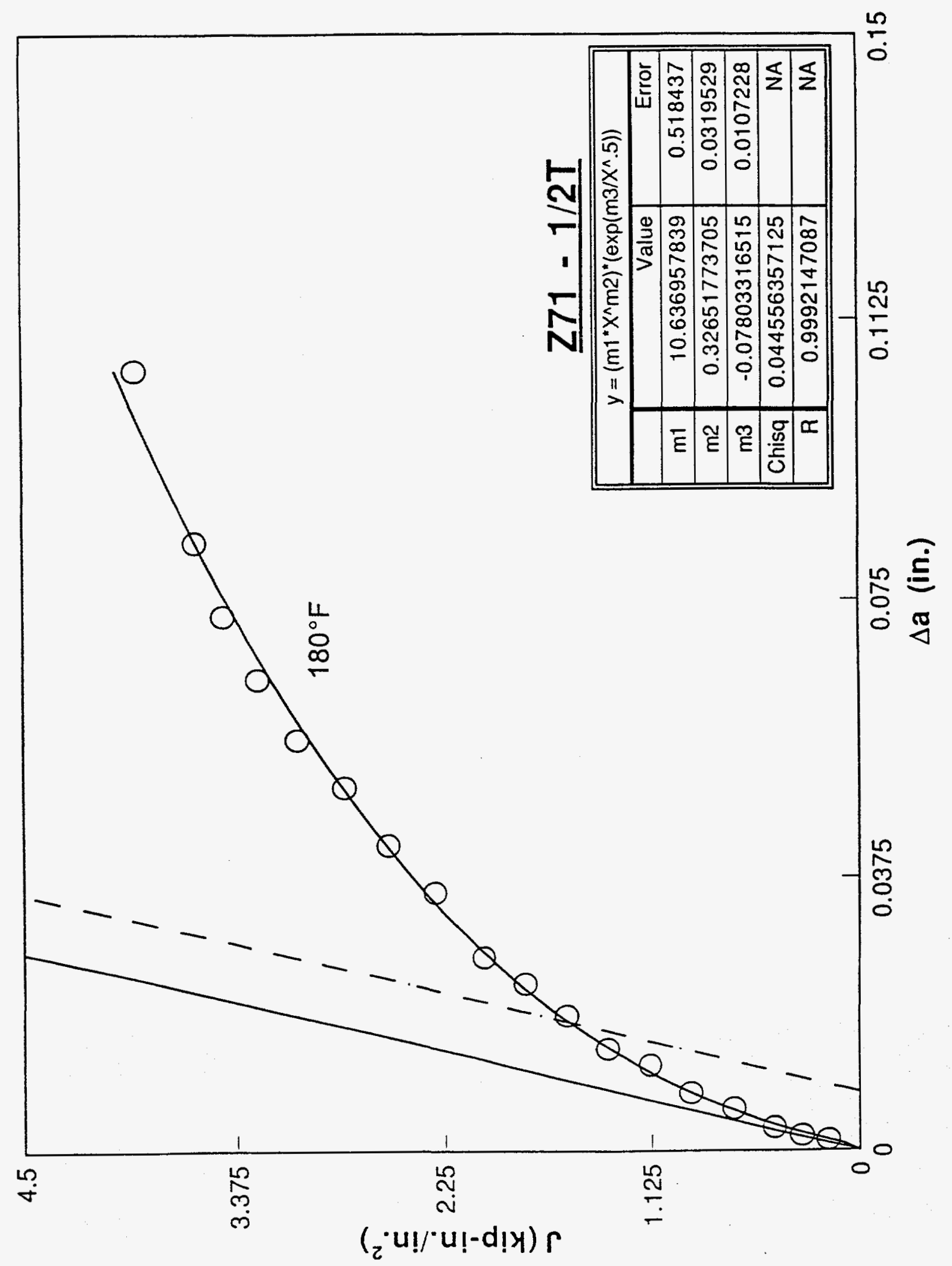




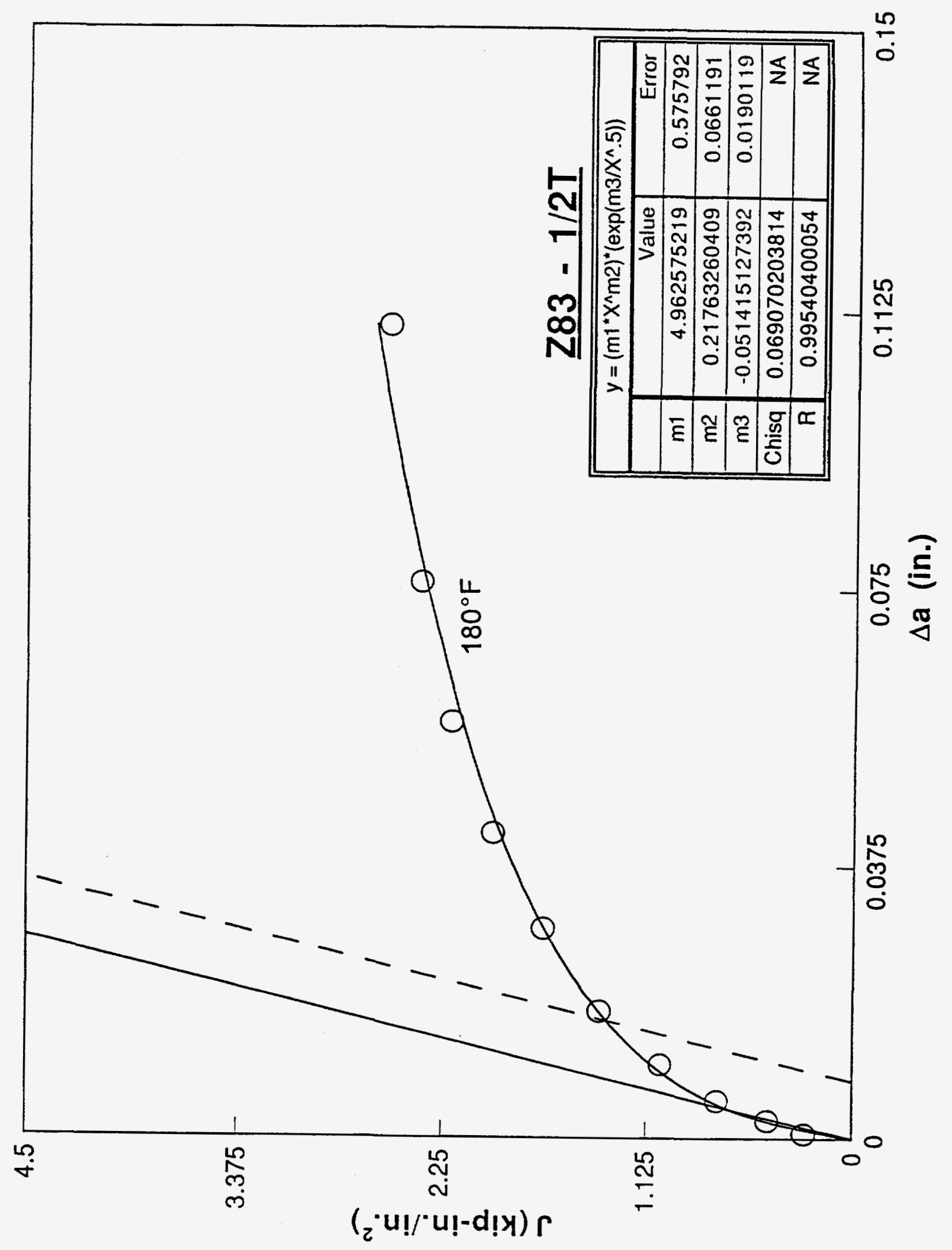




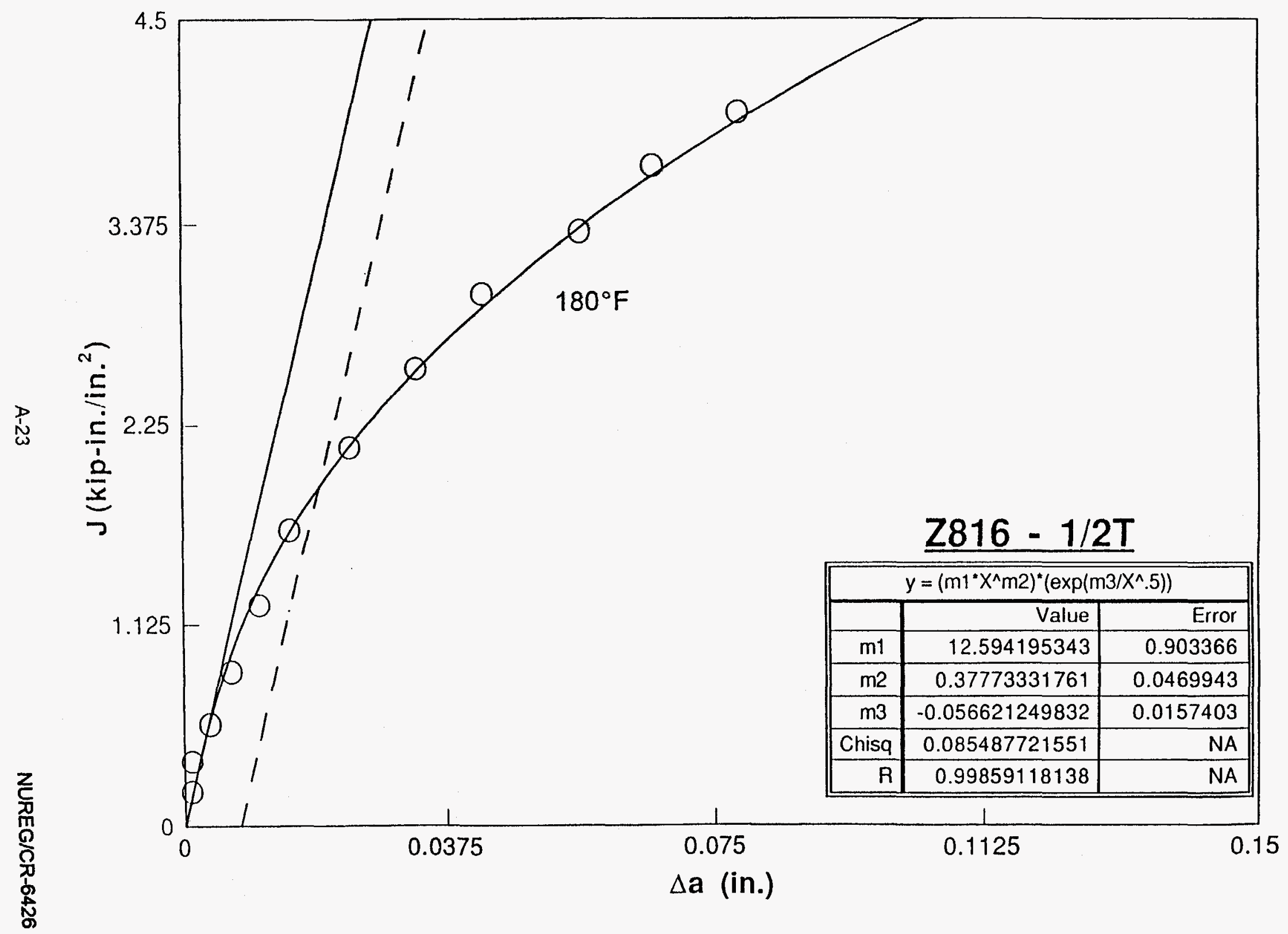




\section{Appendix B}

Selected J-R Curves Representing Typical Values of T Slope, $\mathrm{J}_{0.1}$, and $\mathrm{J}_{\mathrm{lc}}$ from Table 8 


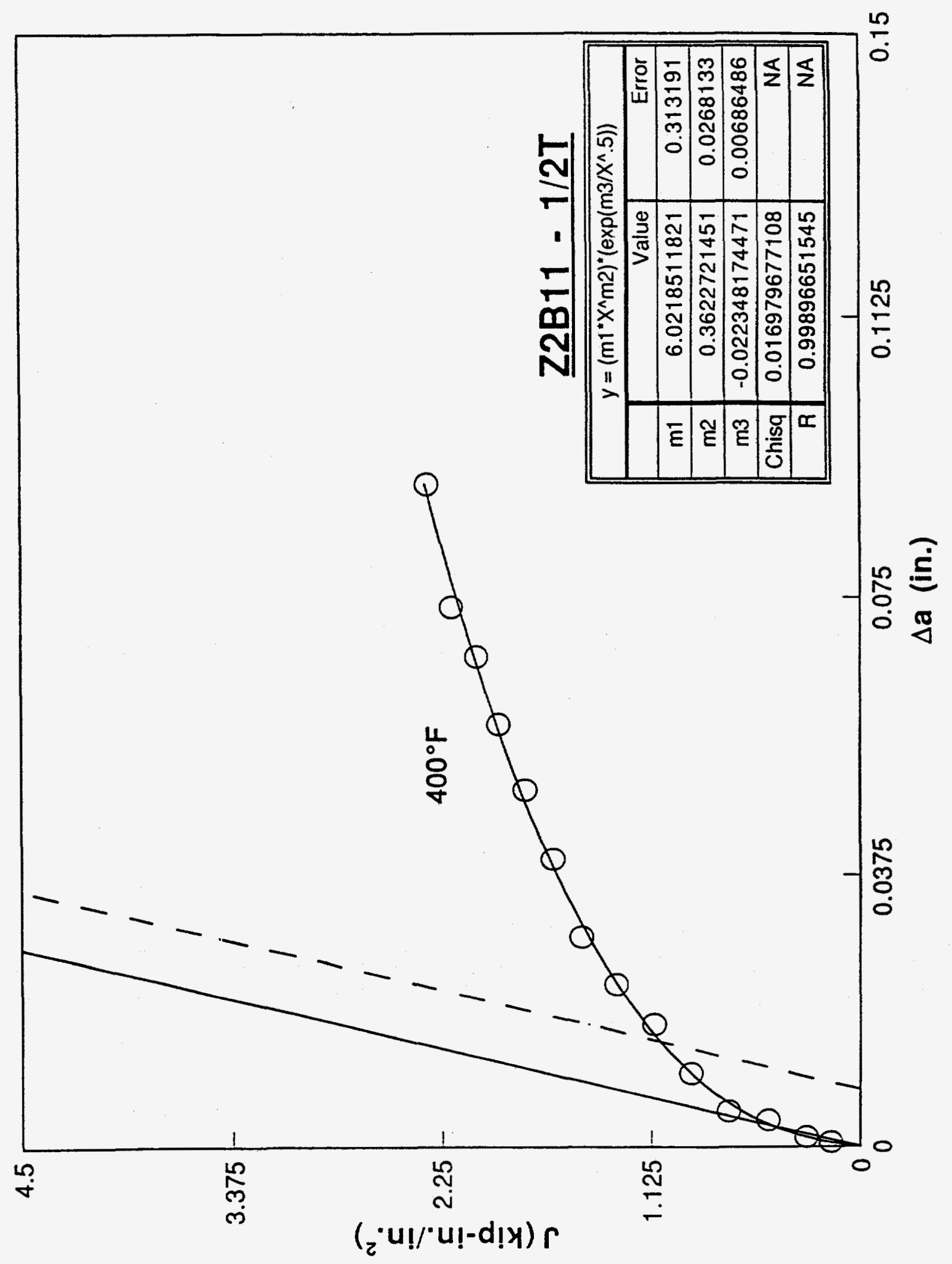




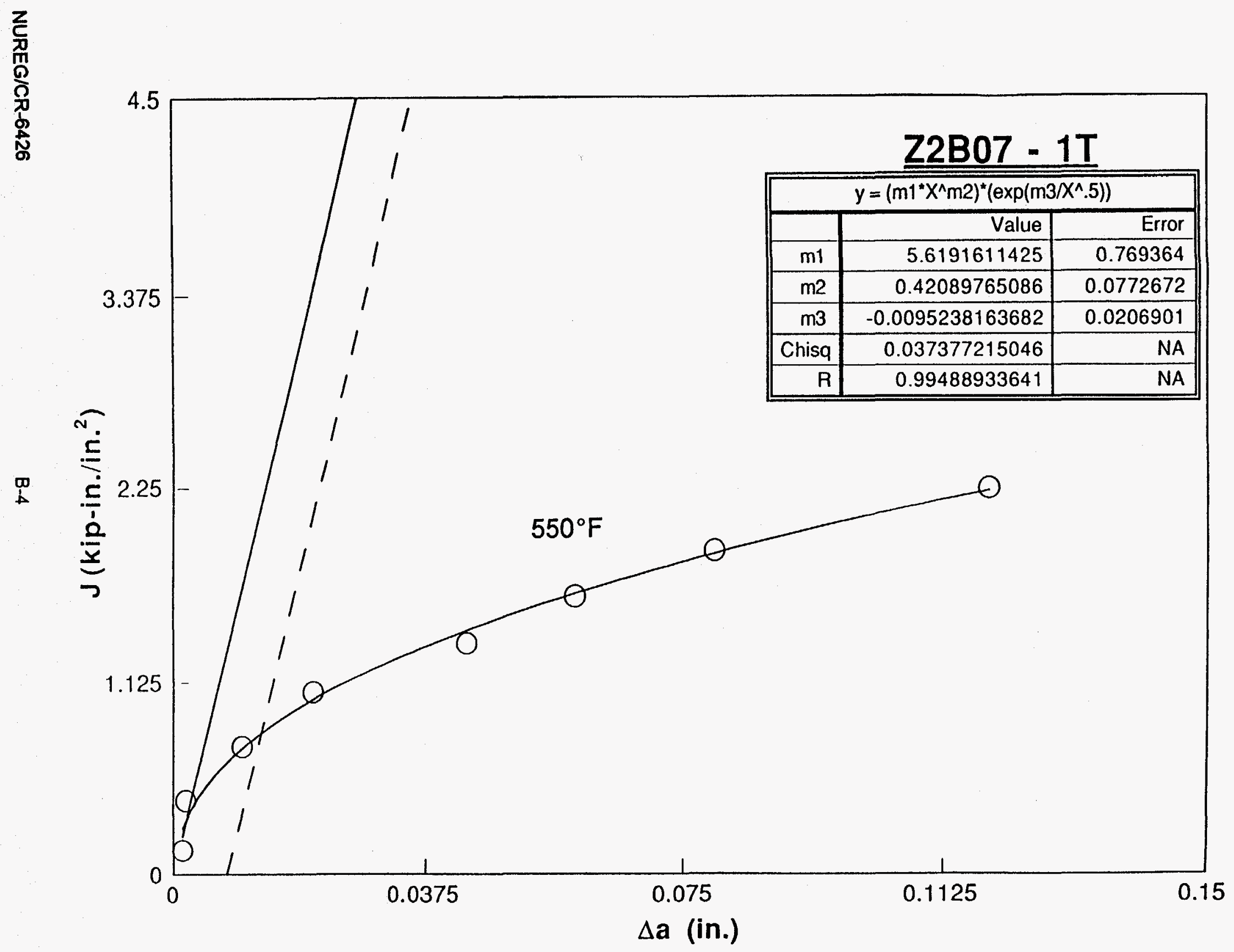




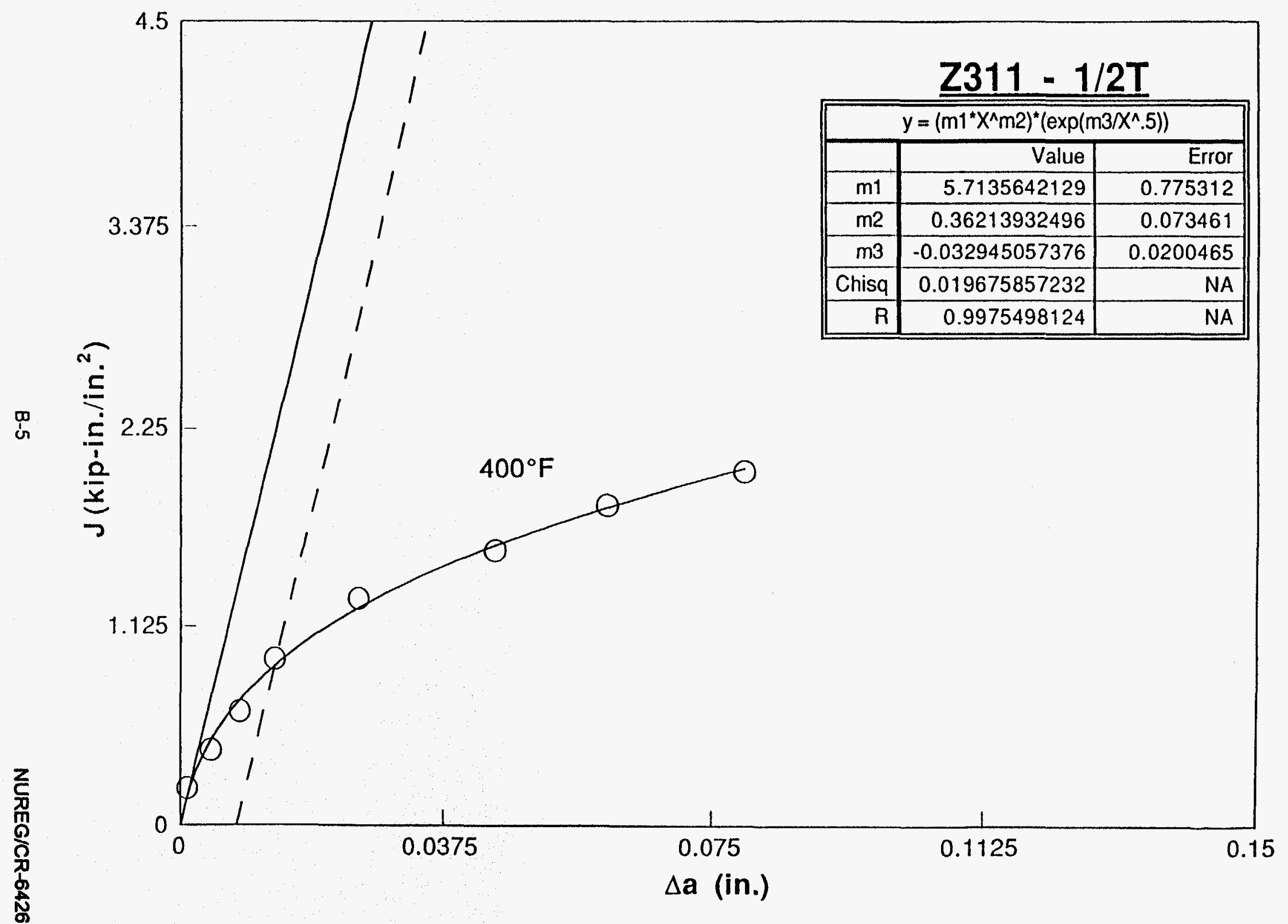




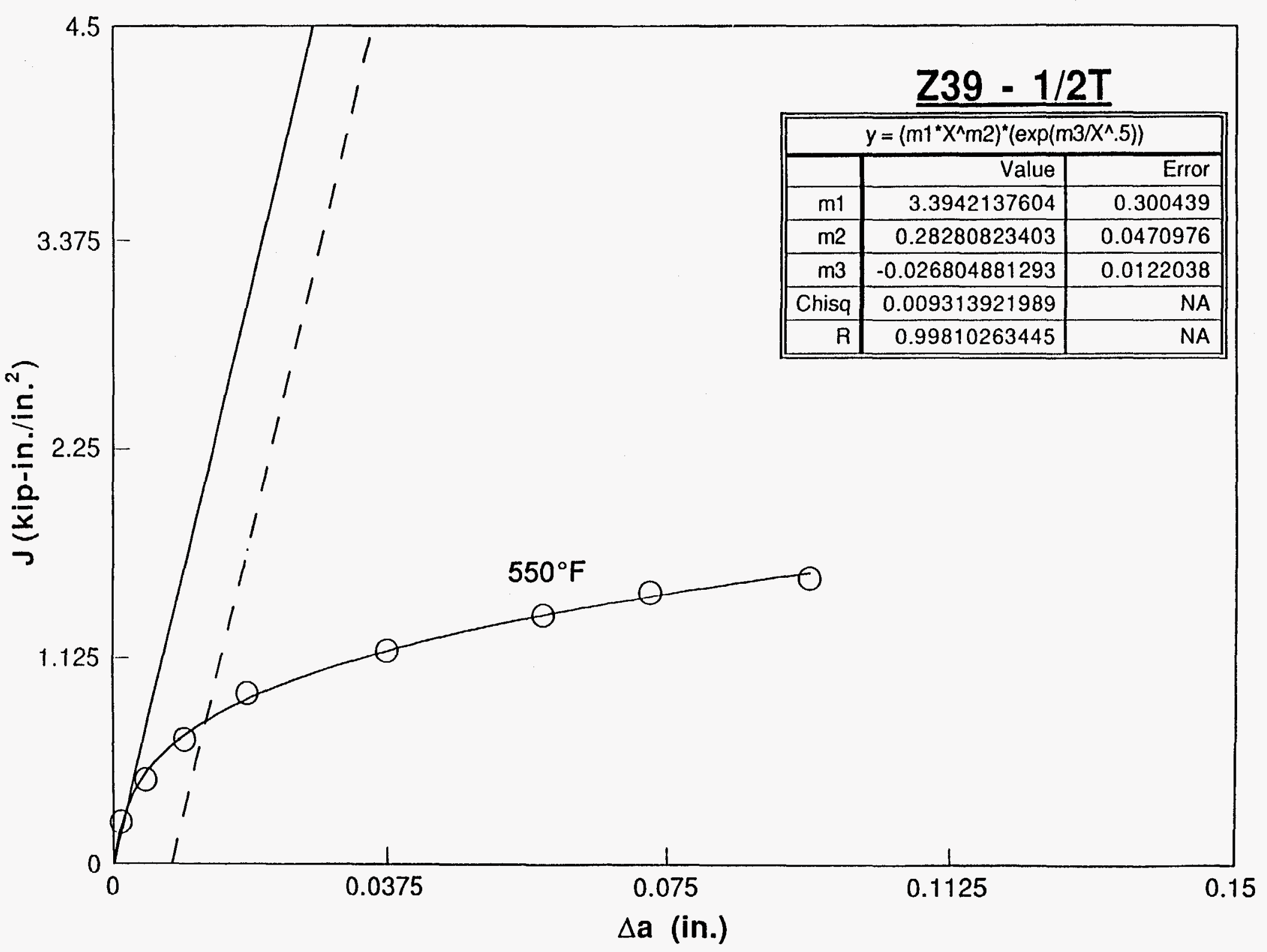




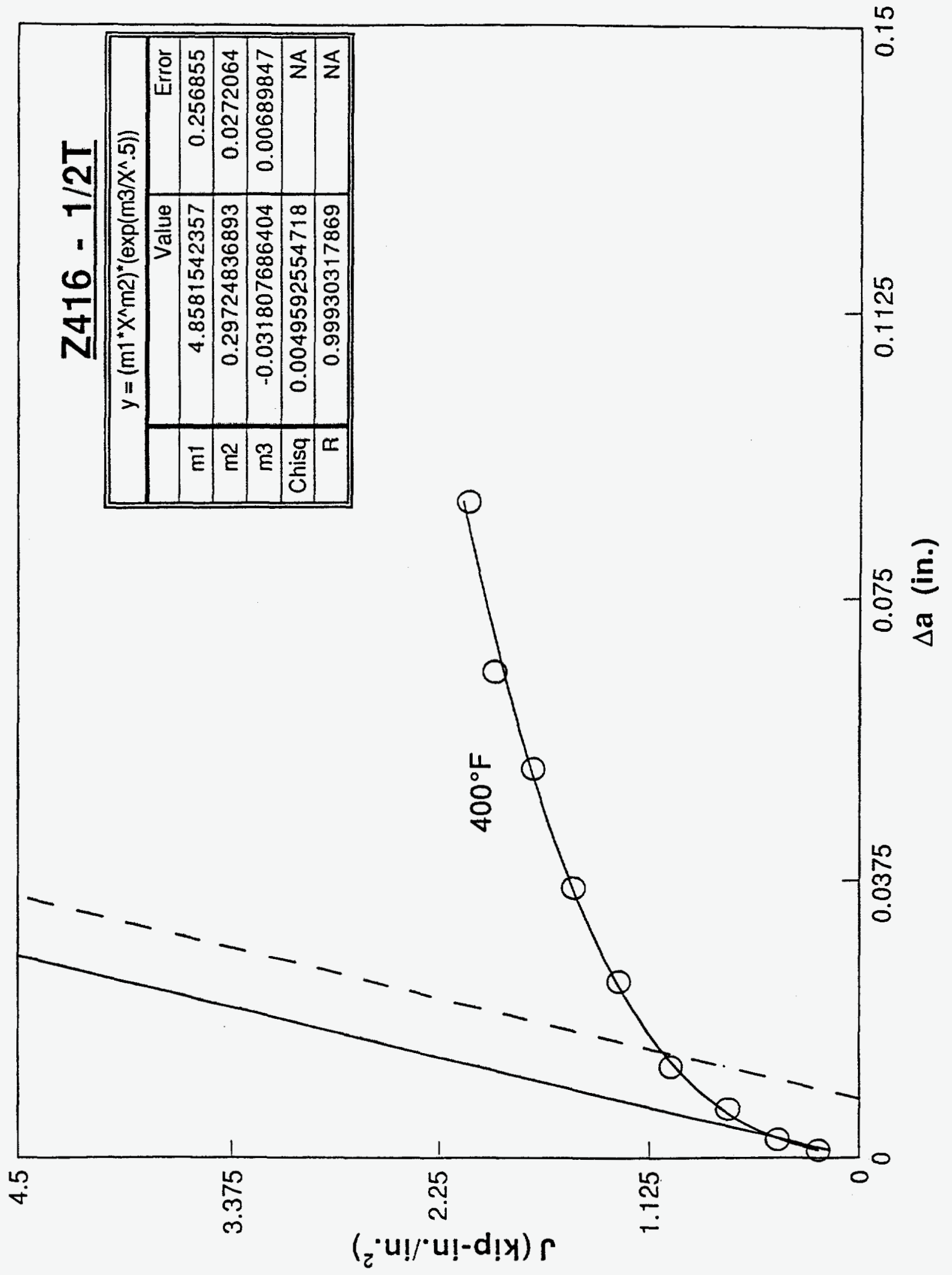




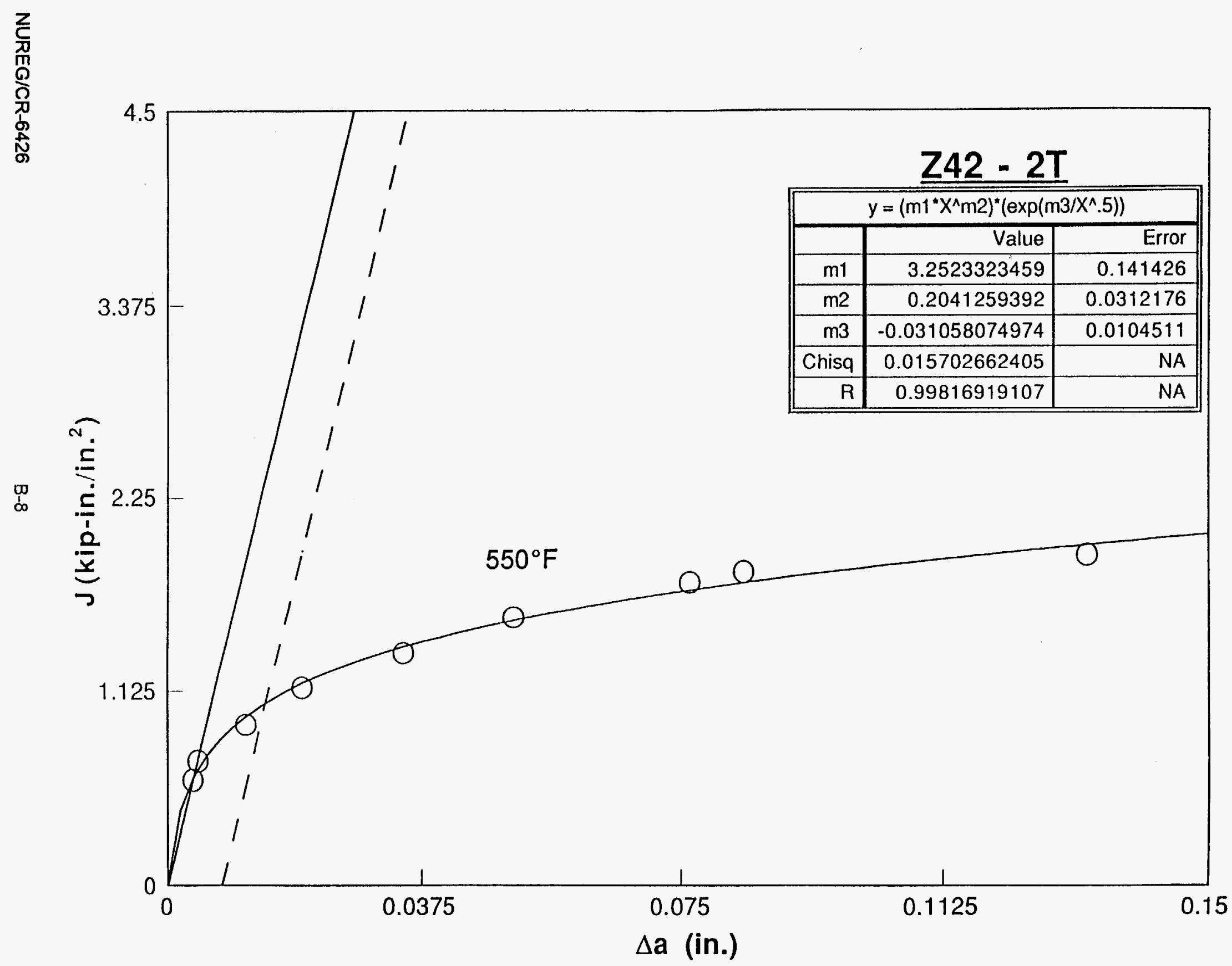




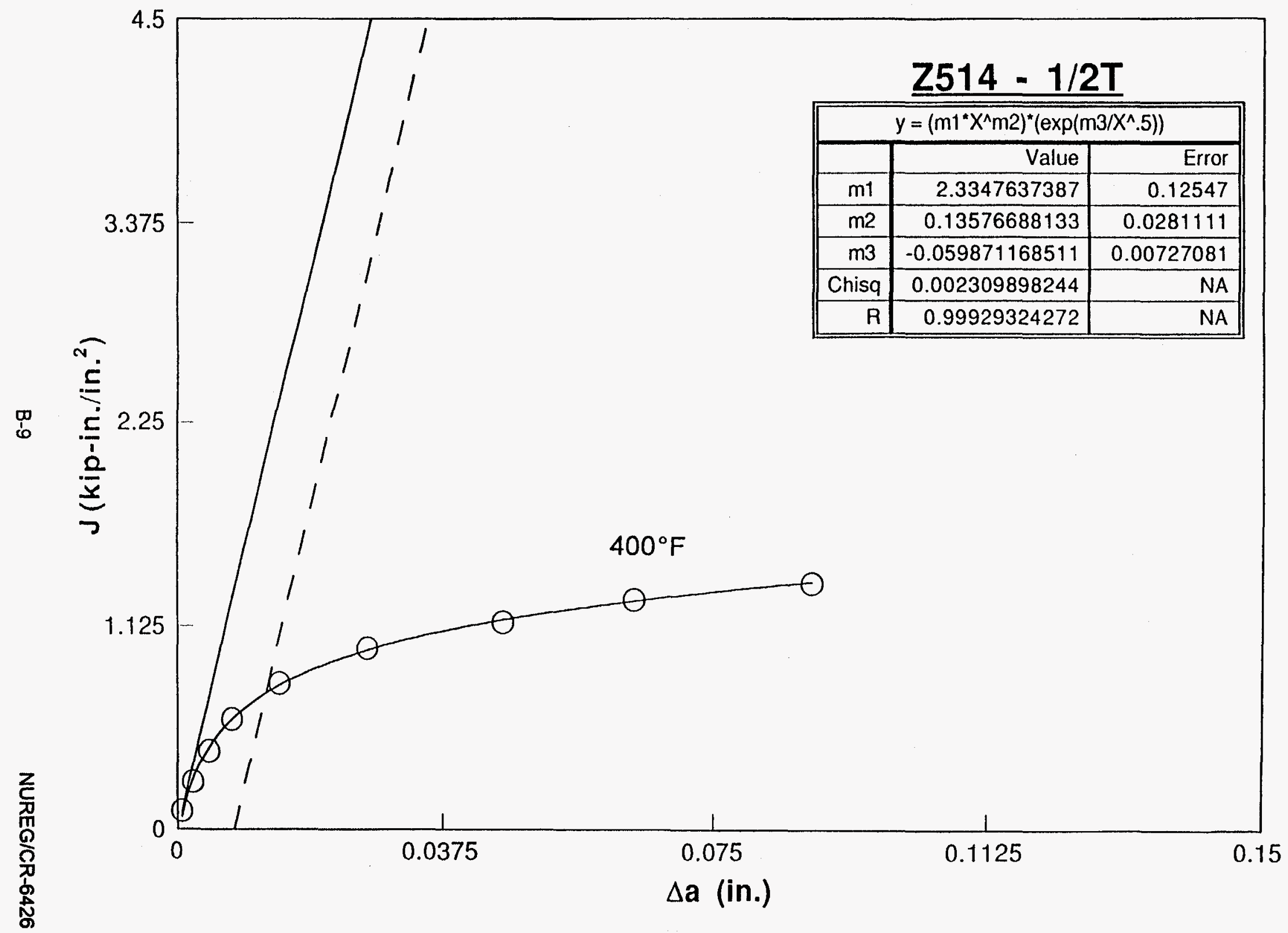




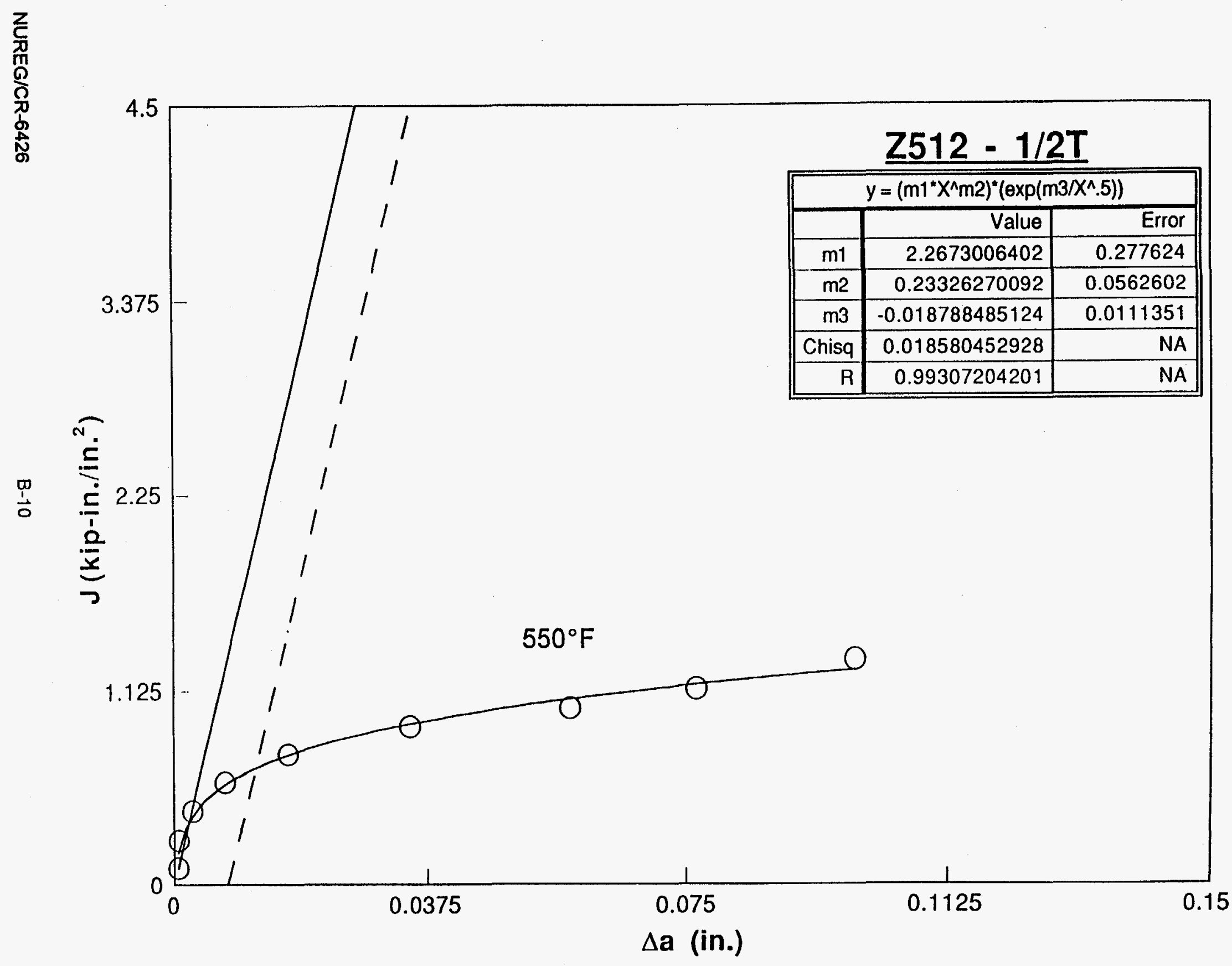




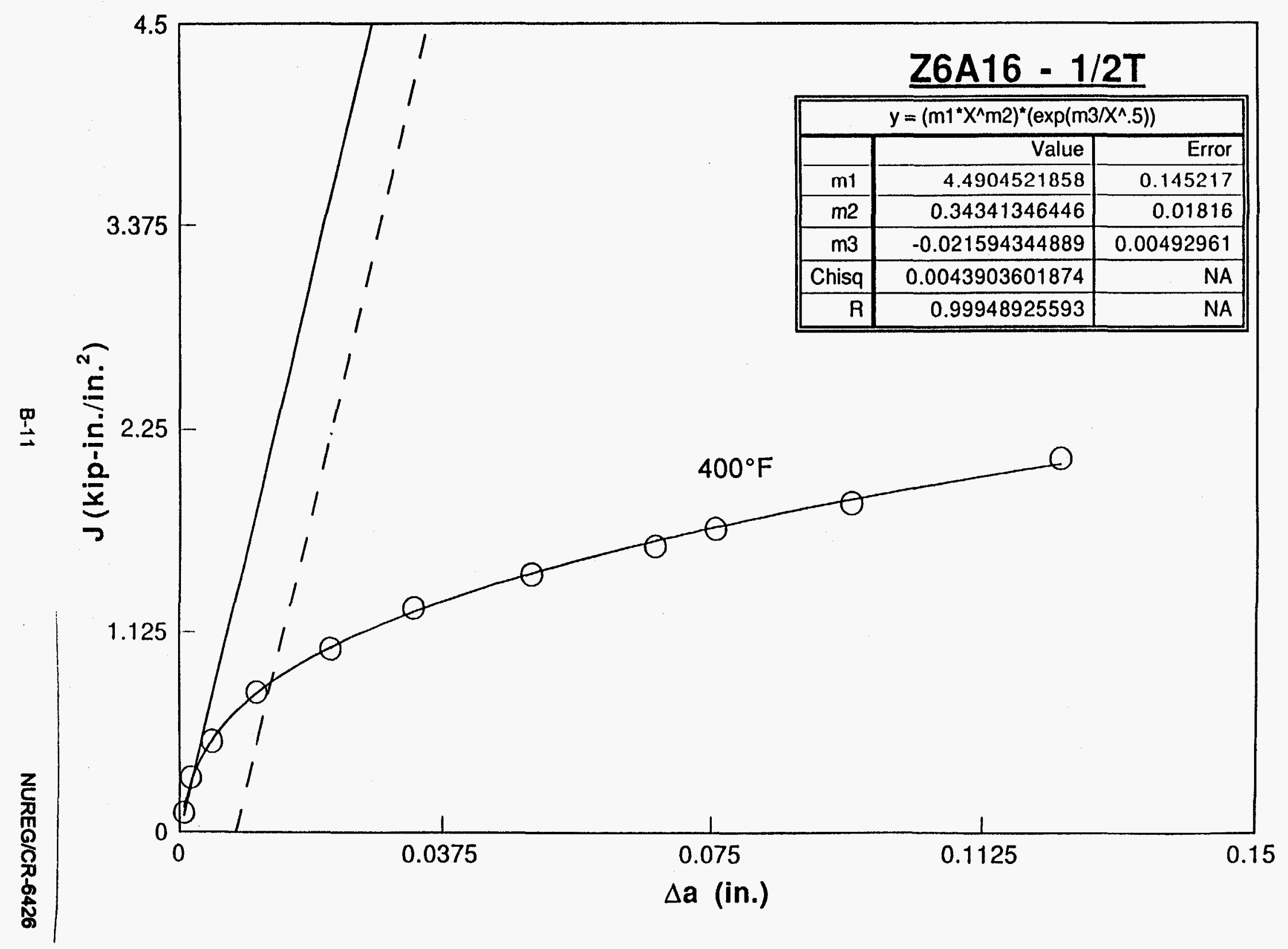




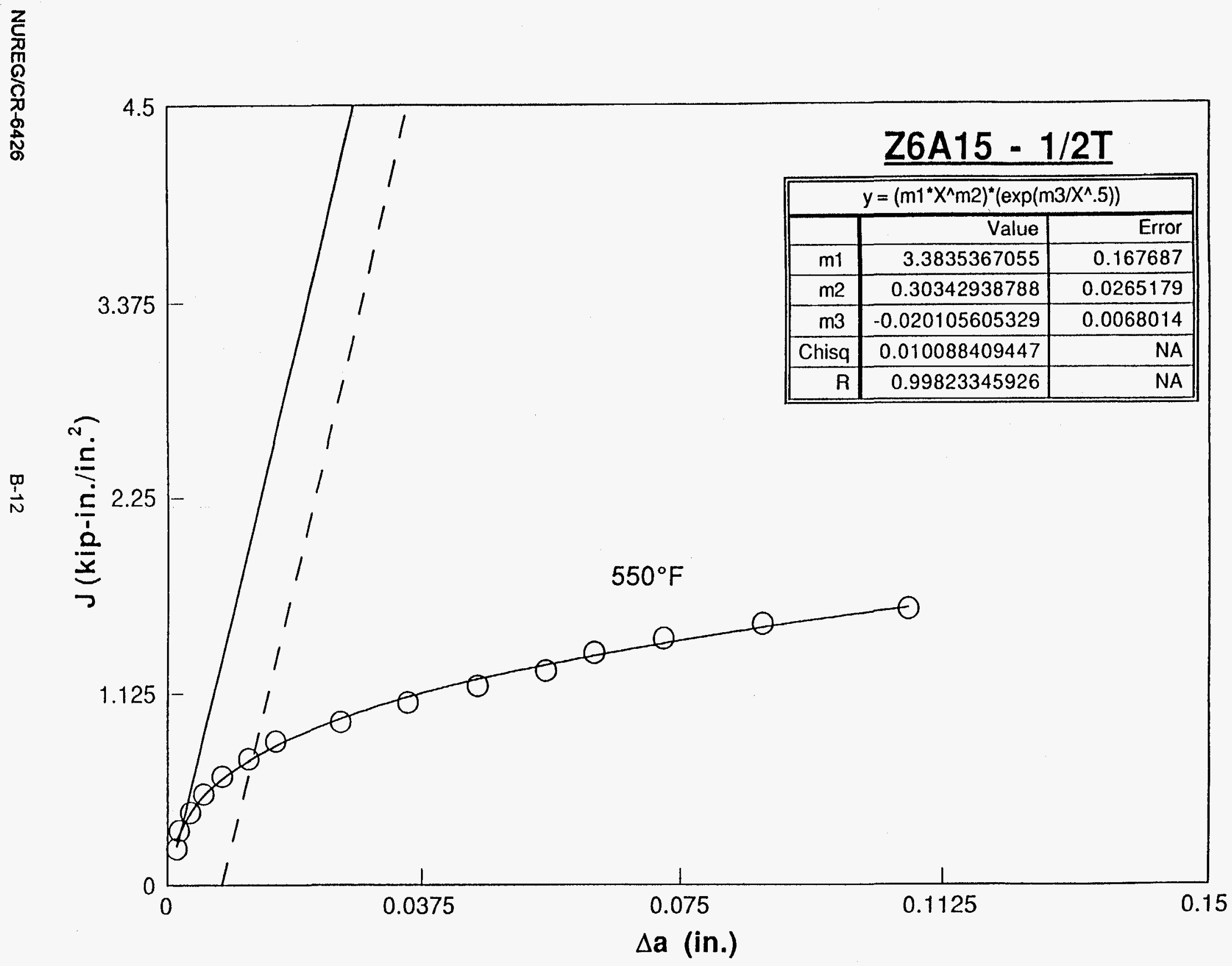




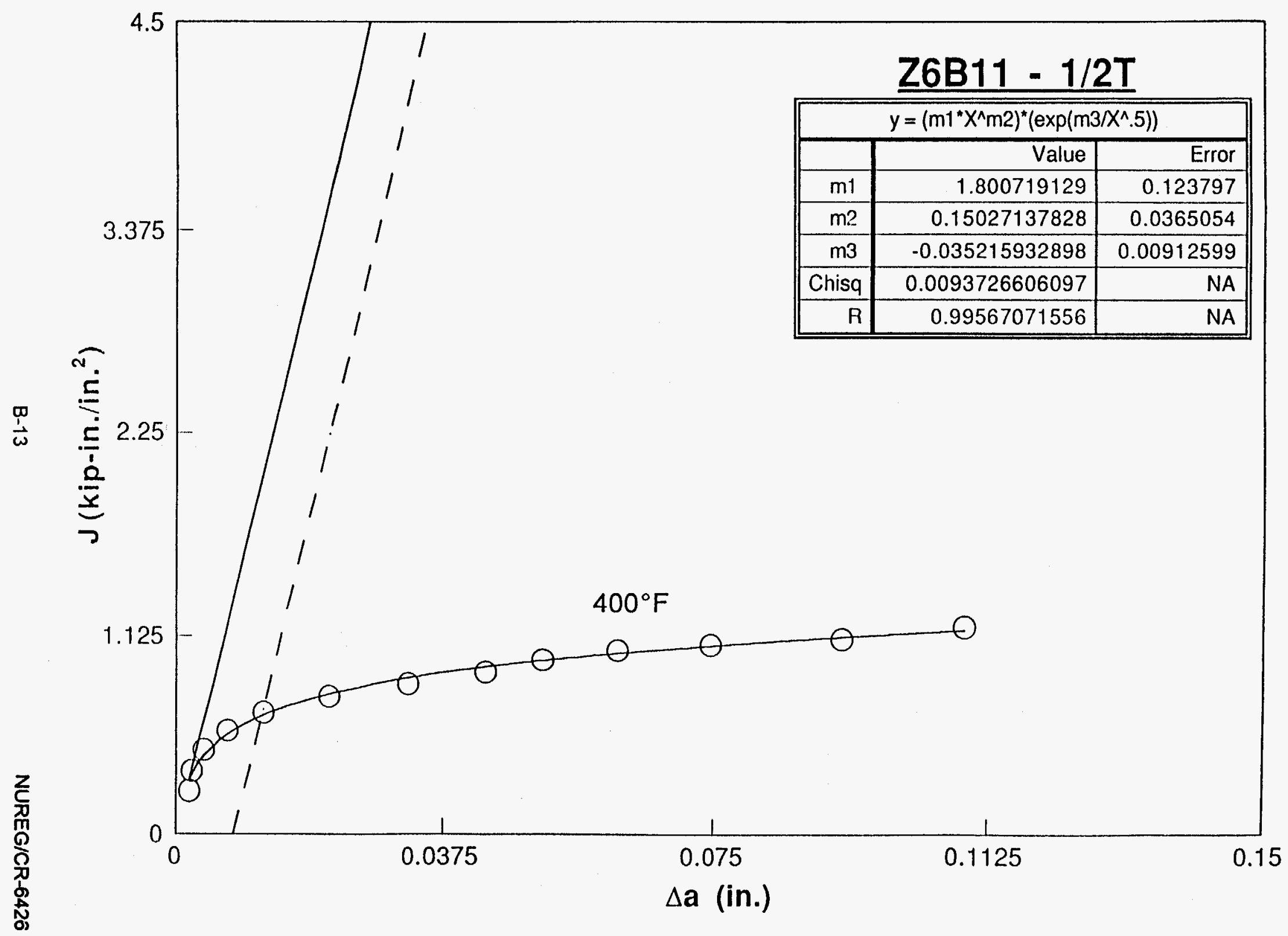




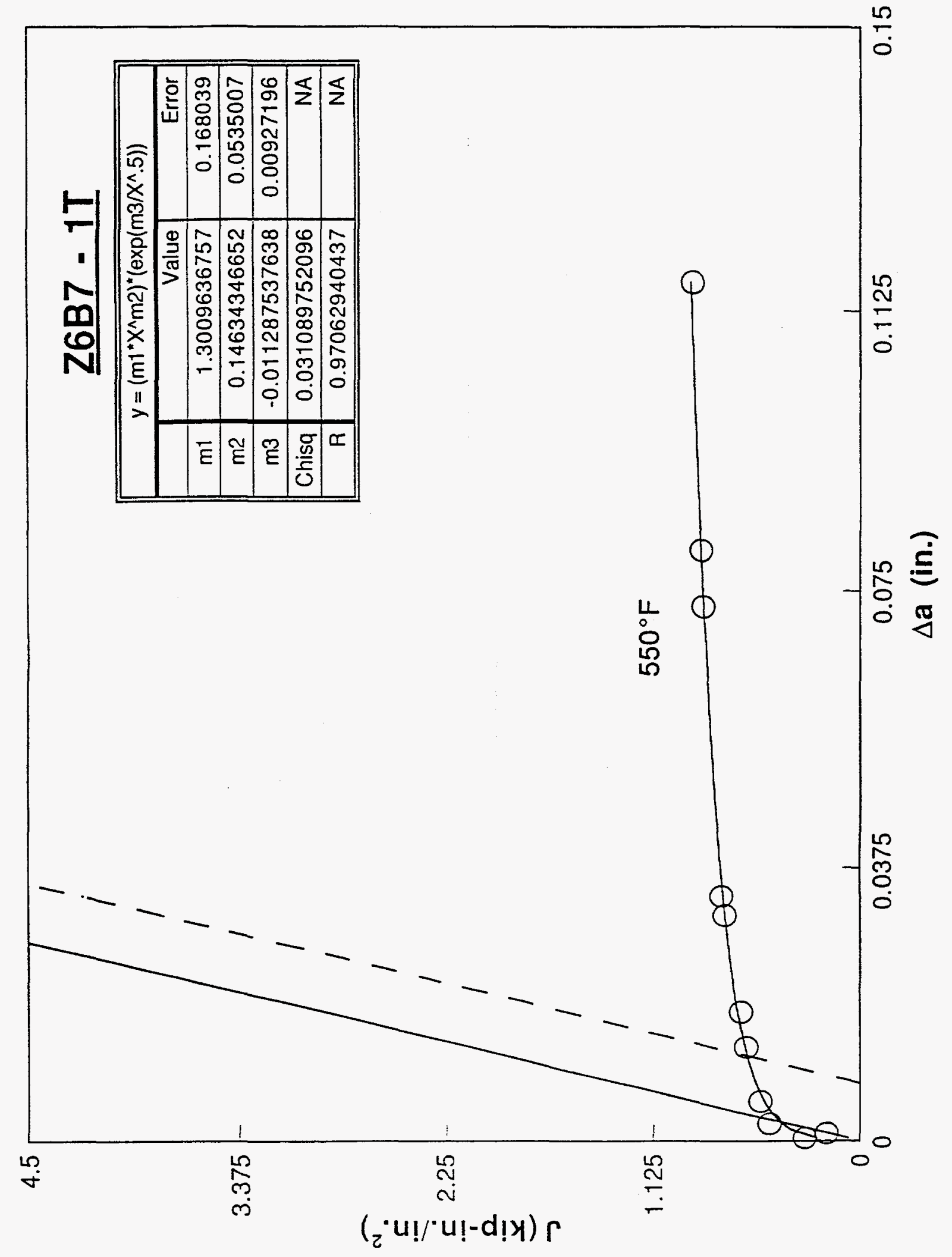




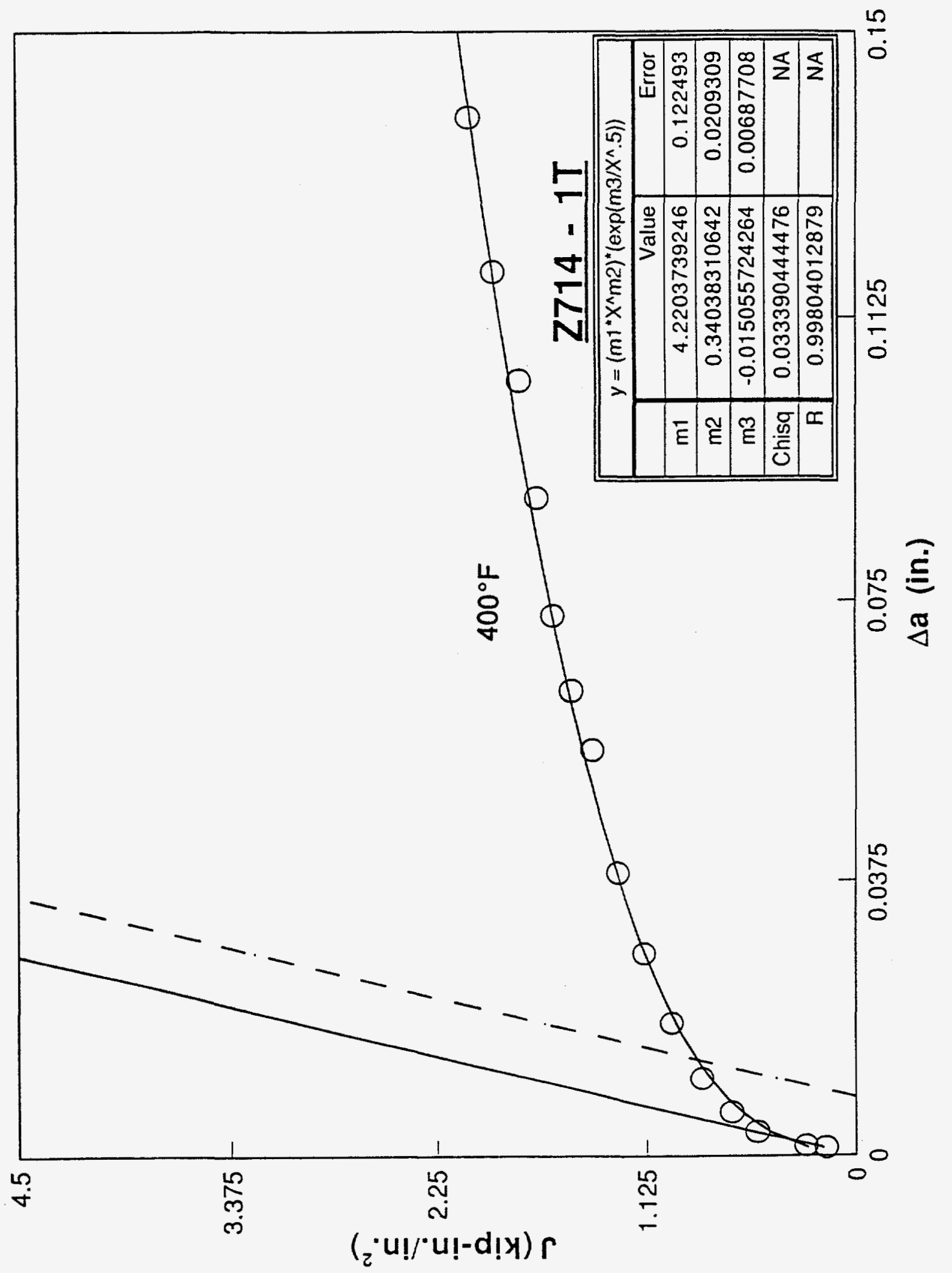

B-15 


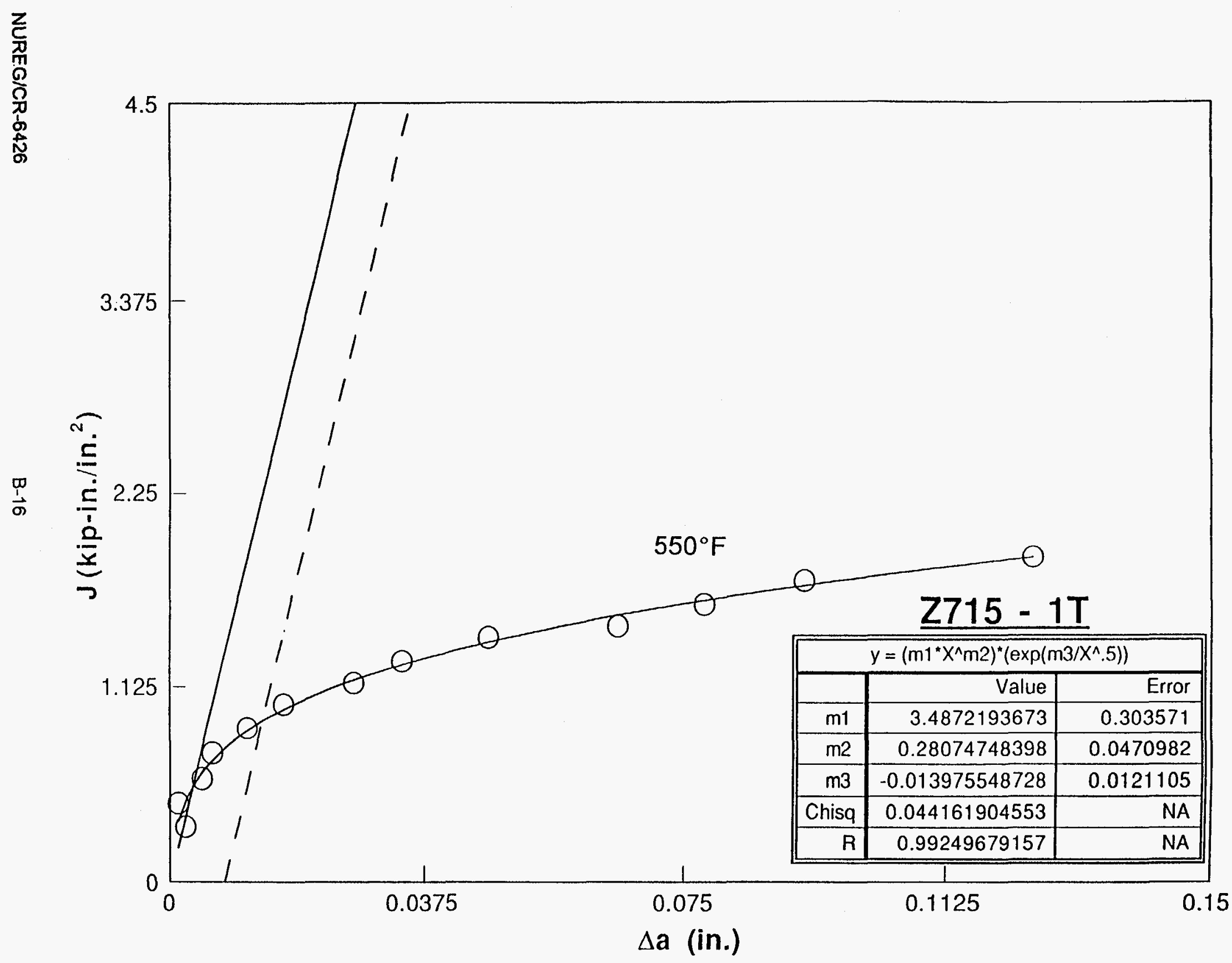




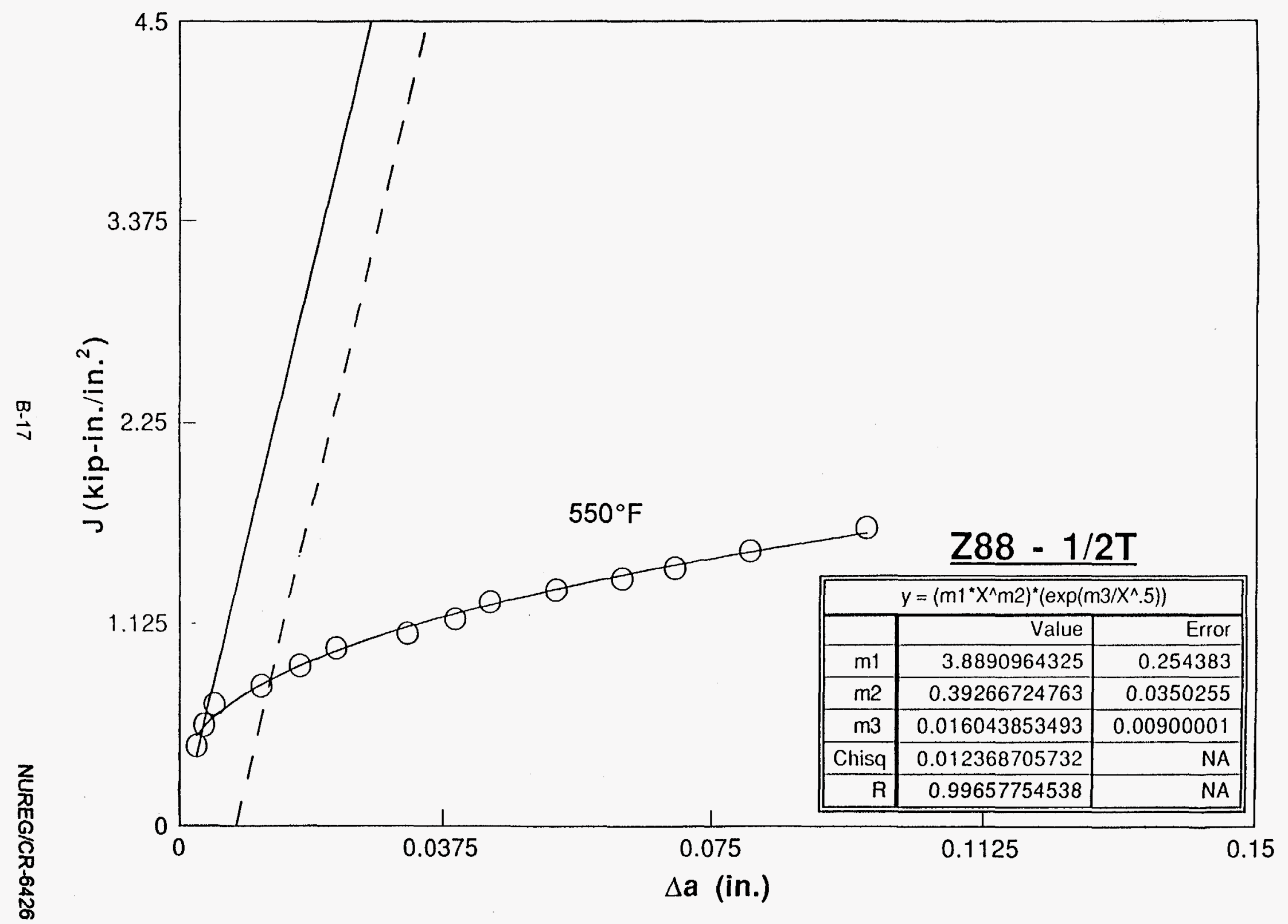




\section{Internal Distribution}

1.

2.

3.

4.

5.

6.

7.

8.

9.

10.

11.

12.

13.

14.

15-19.

20.
D. J. Alexander

B. R. Bass

J. W. Bryson

W. R. Corwin

W. G. Craddick

D. F. Craig

T. L. Dickson

E. C. Fox

H. W. Hayden, Jr.

J. J. Henry, Jr.

S. K. Iskander

W. F. Jackson

J. A. Keeney

R. L. Klueh

E. T. Manneschmidt

W. J. McAfee
21-25.

26.

27-29.

30.

31-33.

34.

35.

36.

37-41.

42.

43.

44.

45.

46-47.

48-49.

50.
D. E. McCabe

J. G. Merkle

R. K. Nanstad

D. J. Naus

W. E. Pennell

C. E. Pugh

M. A. Sokolov

R. E. Stoller

R. L. Swain

R. W. Swindeman

ORNL Patent Section

Central Research Library

Document Reference Section

M\&C Records Office

Laboratory Records

Laboratory Records - RC

\section{External Distribution}

51. R. D. Thompson, Office of Administration, Division of Contracts, U. S. Nuclear Regulatory Commission, Mailstop T7 12, Washington, DC, 20555-0001

52. B. J. Elliot, Division of Engineering, Office of Nuclear Reactor Regulation, Mailstop O7 D4, U.S. Nuclear Regulatory Commission, Washington, DC 20555-0001

53. J. R. Strosnider, Division of Engineering, Office of Nuclear Reactor Regulation, Mailstop O7 D4, U.S. Nuclear Regulatory Commission, Washington, DC 20555-0001

54. K. R. Wichman, Division of Engineering, Office of Nuclear Reactor Regulation, Mailstop O7 D4, U.S. Nuclear Regulatory Commission, Washington, DC 20555-0001

55. M. A. Mitchell, Division of Engineering Technology, Office of Nuclear Reactor Regulation, Mailstop O7 D4, U.S. Nuclear Regulatory Commission, Washington, DC 20555-0001

56. E. M. Hackett, EMMEB/DET/RES, Mailstop T10 E10, U.S. Nuclear Regulatory Commission, Washington, DC 20555-0001

57. R. E. Johnson, EMMEB/DET/RES, U.S. Nuclear Regulatory Commission, Mailstop T10 E10, Washington, DC 20555-0001

58-59. S. N. Malik, EMMEB/DET/RES, U.S. Nuclear Regulatory Commission, Mailstop T10 E10, Washington, DC 20555-0001

60. M. E. Mayfield, EMMEB/DET/RES, U.S. Nuclear Regulatory Commission, Mailstop T10 E10, Washington, DC

61. G. C. Millman, Division of Engineering Technology, U.S. Nuclear Regulatory Commission, Mailstop T10 E10, Washington, DC 20555-0001

62. L. C. Shao, Division of Engineering Technology, U.S. Nuclear Regulatory Commission, Mailstop T10 D20, Washington, DC 20555-0001

63. M. G. Vassilaros, EMMEB/DET/RES, U.S. Nuclear Regulatory Commission, Mailstop T10 E10, Washington, DC 20555-0001

64. J. W. Dally, Department of Mechanical Engineering, University of Maryland, College Park, Maryland 20742

65. E. Eason, Modeling and Computing Services, Suite 105, 6400 Lookout Road, Boulder, Colorado 80301

66. J. D. Landes, University of Tennessee, Engineering Science and Mechanics, 310 Perkins Hall, Knoxville, Tennessee 37996-2030

67. R. E. Link, U.S. Naval Academy, Mechanical Engineering Department, Mailstop 11C, 590 Hollway Road, Annapolis, Maryland 21402 


\section{External Distribution (Continued)}

68. J. A. Joyce, U.S. Naval Academy, Mechanical Engineering Department, MS 11C, 590 Hollway Road, Annapolis, Maryland 21402

69. T. Caine, General Electric Nuclear Energy, Mail Code 747, 175 Curtner Avenue, San Jose, California 95125

70. M. L. Hamilton, Battelle Pacific Northwest National Laboratory, Structural Materials Research Section, MS P8-15, Battelle Boulevard, P. O. Box 999, Richland, Washington 99352

71. W. A. Van der Sluys, Babcock and Wilcox, R\&D Division, 1562 Beeson Street, Alliance, Ohio 44601

72. G. R. Irwin, Department of Mechanical Engineering, University of Maryland, College Park, Maryland 20742

73. L. A. James, Westinghouse Bettis Lab., P. O. Box 79, ZAP 13A, 814 Pittsburgh McKeesport Blvd., West Mifflin, Pennsylvania 15122

74. C. F. Shih, Box D, Division of Engineering, Brown University, Providence, Rhode Island, 02912

75. R. Dodds, 3140 Newmark Laboratory, 205 North Matthews, Urbana, Illinois 61801

76. R. Fields, National Inst. of Standards and Technology, Bldg. 223, B144, Gaithersburg,, Maryland 20899

77. W. L. Fourney, Department of Mechanical Engineering, University of Maryland, College Park, Maryland 20742

78. S. R. Martin, DOE-ORO, P. O. Box 2008, Oak Ridge, Tennessee 37831-6269

79. S. T. Rolfe, The University of Kansas, Lawrence, Kansas 66045-2235

80. A. R. Rosenfield, Battelle Columbus Division, Columbus, Ohio 43201

81. C. W. Schwartz, Department of Civil Engineering, University of Maryland, College Park, Maryland 20742

82. E. T. Wessel, 312 Wolverine, Lake Region Mobile Home Village, Haines City, Florida 33844

83. R. L. Tregoning, Code-614, Fatigue and Fracture Branch, NSWC-CD, 9500 McArthur Blvd., Bethesda, MD 20817

84. R. O. Hultgren, ORNL Site Manager, Office of Energy Research and Development, DOE-OR, P. O. Box 2008, Oak Ridge, TN 37831-6269

85-86. Office of Scientific and Technical Information, P. O. Box 62, Oak Ridge, Tennessee 37831 
(See instructions on the reverse)

2. TITLE AND SUBTITLE

ORNL -6892

Ductile Fracture Toughness of Modified A 302 Grade B Plate Materials, Data Analysis

VoT . 1

3. DATE REPORT PUBLISHED

4. FIN OR GRANT NUMBER B0119

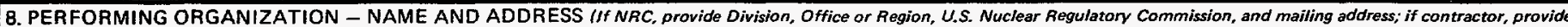
name and mailir.g address.)

Oak Ridge National Laboratory

Oak Ridge, Tennessee 37831-6285

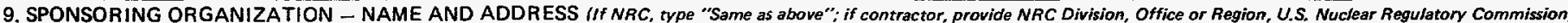
and mailing address.

Division of Engineering Technology

Office of Nuclear Regulatory Research

U.S. Nuclear Regulatory Commission

Washington, DC 20555-0001

10. SUPPLEMENT ARY NOTES

S. N. M. Malik, NRC Project Manager

11. ABSTRACT (200 words or less)

The objective of this work was to develop ductile fracture toughness data in the form of J-R surves for modified A 302 grade B plate materials typical of those used in fabricating reactor pressure vessels. A previous experimental study at Materials Engineering Associates, Lanham, Maryland, on one particular heat of A 302 grade B plate showed decreasing J-R curves with increased specimen thickness. This characteristic has not been observed in numerous tests made on the more recent production mterials of A 533 grade B and A 508 class 2 pressure vessel steels. It was unknown if the departure from norm for the MEA material was a gneric characteristic for a11 heats of A 302 grade B steels or just unique to that one particular plate.

12. KEY WORDS/DESCR!PTORS (List words or phrases that will assist researchers in locating the report.

J-R Crack-Growth Resistance Curves

Ductile Fracture

A 302 Grade B Steel Plate

Reactor Pressure Vessel Steel

Charpy Low Upper Shelf Energy

ASTM E 813-87 and E. 1152-89 Test Standards

13. AVAILABILITY STATEMENT

Unlimited

14. SECURITY CLASSIFICATION

(This $F$, , p $)$

Unclassified

Unclassified

15. NUMBER OF PAGES

16. PRICE 